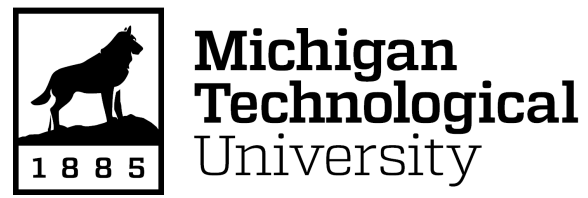

Michigan Technological University Digital Commons @ Michigan Tech

Dissertations, Master's Theses and Master's Reports

2016

\title{
A FAULT TOLERANT, DATA FUSION SYSTEM FOR NAVIGATION APPLICATIONS TO A DUCTED FAN VTOL UAV
}

Jian Lu

Michigan Technological University, jilu@mtu.edu

Copyright 2016 Jian Lu

\section{Recommended Citation}

Lu, Jian, "A FAULT TOLERANT, DATA FUSION SYSTEM FOR NAVIGATION APPLICATIONS TO A DUCTED FAN VTOL UAV", Open Access Master's Report, Michigan Technological University, 2016.

https://doi.org/10.37099/mtu.dc.etdr/98

Follow this and additional works at: https://digitalcommons.mtu.edu/etdr

Part of the Electrical and Electronics Commons 


\title{
A FAULT TOLERANT, DATA FUSION SYSTEM FOR NAVIGATION APPLICATIONS TO A DUCTED FAN VTOL UAV
}

\author{
By \\ Jian Lu
}

\author{
A REPORT \\ Submitted in partial fulfillment of the requirements for the degree of \\ MASTER OF SCIENCE \\ In Electrical Engineering \\ MICHIGAN TECHNOLOGICAL UNIVERSITY \\ 2016 \\ (C) 2016 Jian Lu
}


This report has been approved in partial fulfillment of the requirements for the Degree of MASTER OF SCIENCE in Electrical Engineering

Department of Electrical \& Computer Engineering

\author{
Report Advisor: Jeffrey B. Burl \\ Committee Member: Nilufer Onder \\ Committee Member: Timothy Havens
}

Department Chair: Daniel R. Fuhrmann 


\section{Table of Contents}

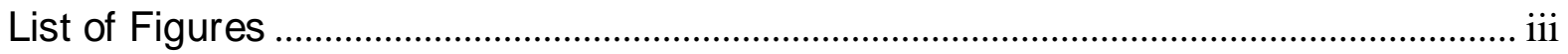

List of Tables

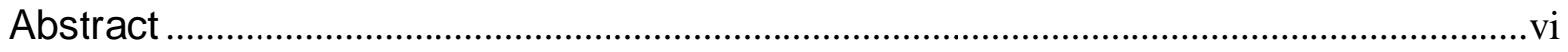

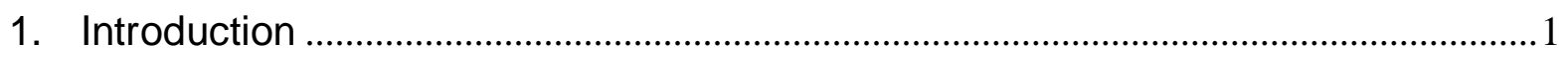

1.1. How to Design a Data Fusion and Fault Tolerant System ...........................

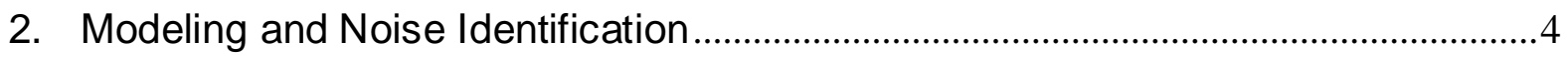

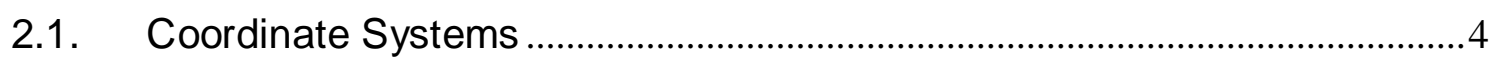

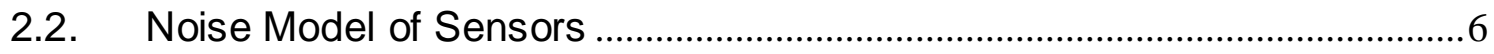

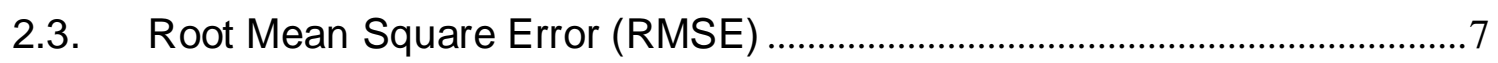

2.4. Measurements for Multiple Sensors .............................................................

2.5. Noise Identify Technique …………………...............................................15

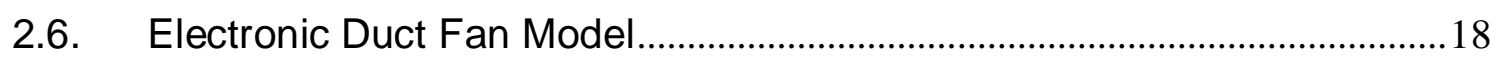

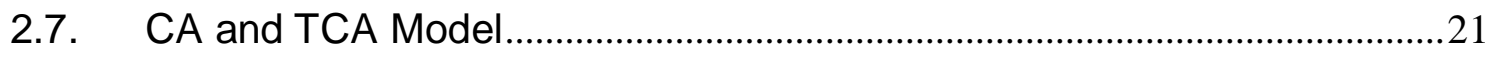

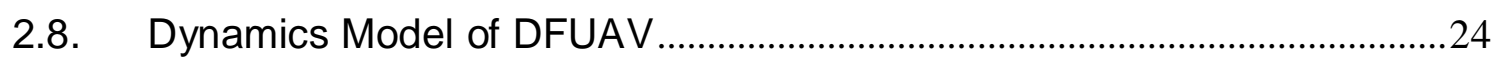

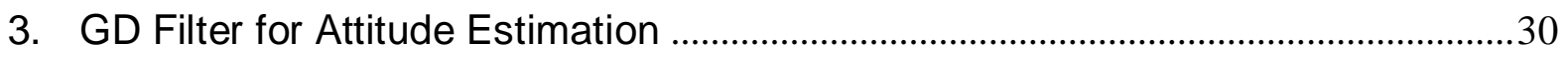

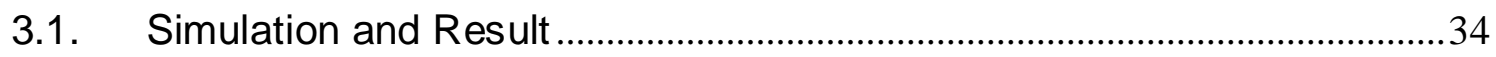

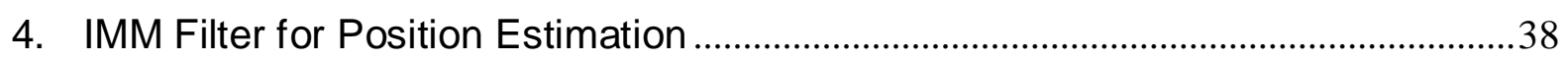

4.1. IMM Filter Description ...................................................................................

4.2. Design of IMM Filter ....................................................................................

4.2.1. IMM Filter Process Framework....................................................................41 
4.2.2. IMM Models Bank

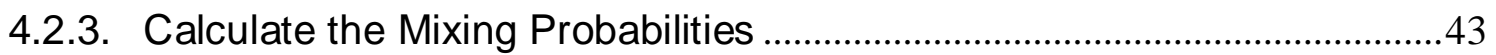

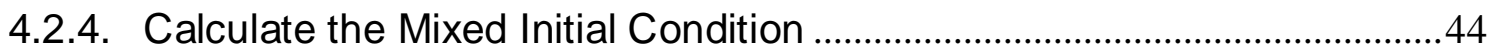

4.2.5. Mode-Matched Filtering and Likelihood Function.........................................44

4.2.6. Update Model Probability .................................................................................

4.2.7. Combine Model- Conditioned Estimates and Covariance...........................46

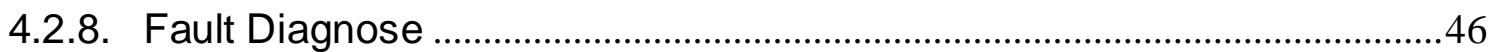

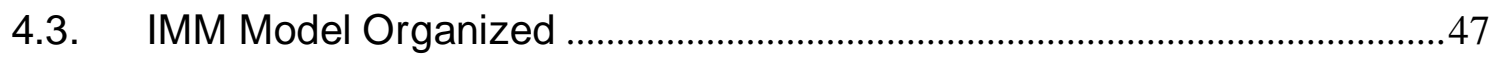

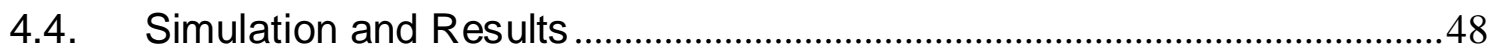

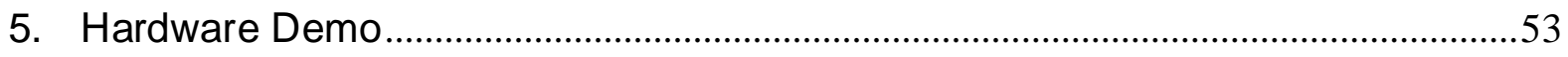

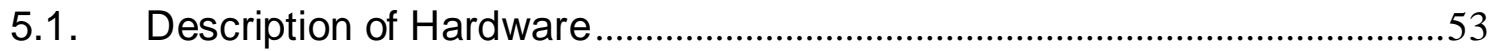

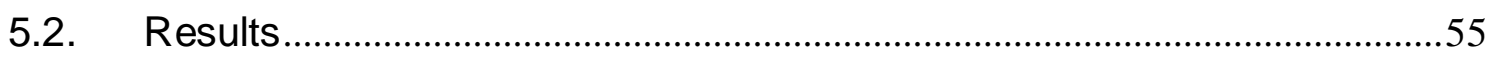

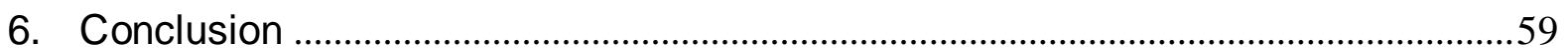

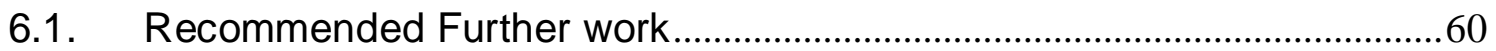

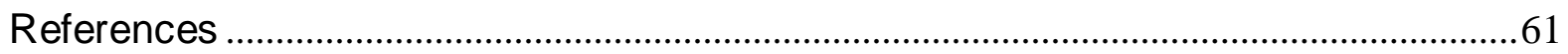




\section{List of Figures}

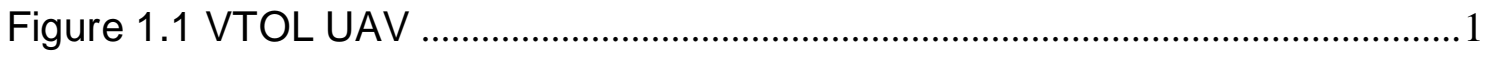

Figure 1.2 VTOL UAV Navigation System Connections ...........................................

Figure 2.1 Normal Sensor Noises Model Structure ..................................................6

Figure 2.2 Inertial Measurement Unit ................................................................

Figure 2.3 GPS Position and Velocity Variance ....................................................13

Figure 2.4 20,000 Sec. Gyroscope Allan Variance Statistics Characteristic .......15

Figure 2.5 Typical Allan Deviation plot for a system ${ }^{[7]}$.........................................16

Figure 2.6 Gyroscope Root Allan Variance ……………......................................17

Figure 2.7 Accelerometer Root Allan Variance ........................................................17

Figure 2.8 Assembly of Electronic Duct Fan system ..............................................18

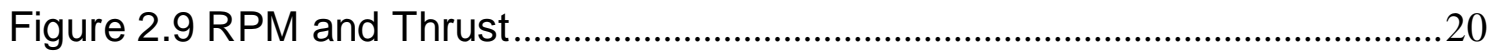

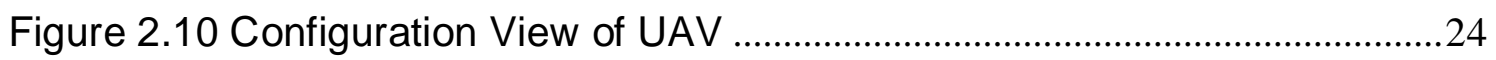

Figure 2.11 Definition of Force and Moment......................................................26

Figure 2.12 Air Flow through the Duct Fans ………….....................................28

Figure 3.1 Gradient Descent Algorithm Block Diagram ............................................32

Figure 3.2 Flight Signal Generator Noise Parameters ...............................................35

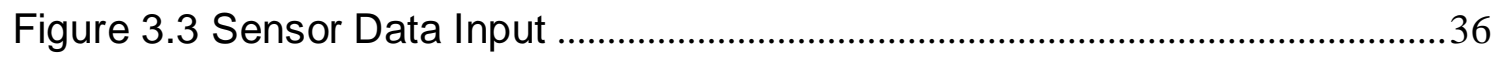

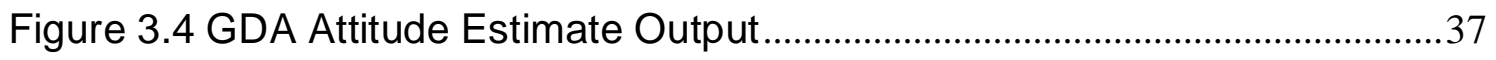

Figure 3.5 RMSE Testing for Different Learning Rates $\beta$........................................37

Figure 4.1 GPB1, GBP2 and IMM frameworks ....................................................40 
Figure 4.2 Block diagram for the IMM Algorithm

Figure 4.3 IMM Model organized structure

Figure 4.4 Speed sensor failures and GPS 1 signal lost describes .48

Figure 4.5 Fault-Tolerance Processing when sensor failures .49

Figure 4.6 Diagnosis information ......................................................................50

Figure 4.7 IMM filter estimation, Dotted line show actual signal ............................51

Figure 4.8 IMM filter position and velocity RMSE ...............................................52

Figure 5.1 VTOL UAV Electronic System Functional Block Diagram ....................53

Figure 5.2 Navigation Computer .............................................................................54

Figure 5.3 Acceleration and Magnetometers PDF ..............................................56

Figure 5.4 Pitch, Roll and Yaw Data from GD Filter ..............................................57

Figure 5.5 GPB 1 filter for multiple GPSs signal mixture ......................................58

Figure 5.6 Google Map path matching with IMM estimation ....................................58 


\section{List of Tables}

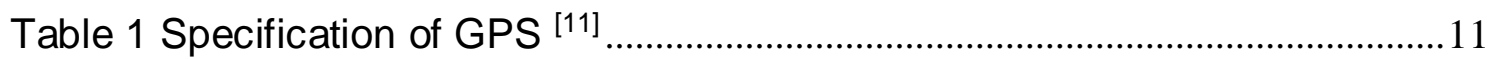

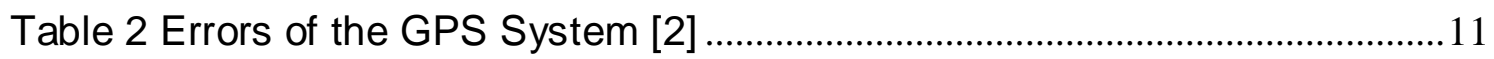

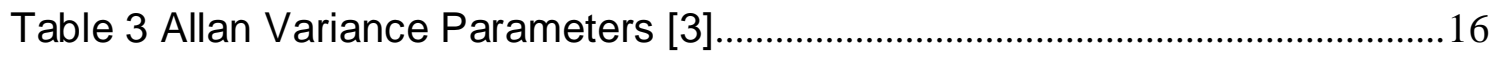

Table 4 Specification of Electronic Duct Fan ........................................................18

Table 5 Specification of Brushless Motor ............................................................19

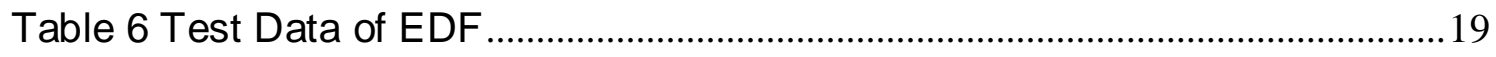

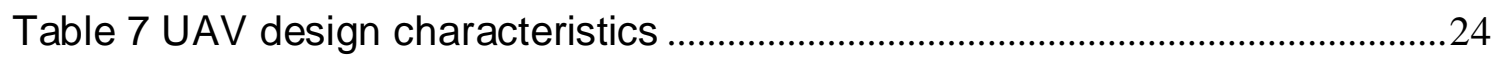

Table 8 Compare different computation complexity with different algorithm .......40

Table 9 Average RMSEs during Non-Maneuvering ................................................40

Table 10 Average Position \& Velocity RMSEs during Maneuver ..............................40

Table 11 Relative Execution Time ........................................................................

Table 12 Compare RMSE in different state ........................................................52

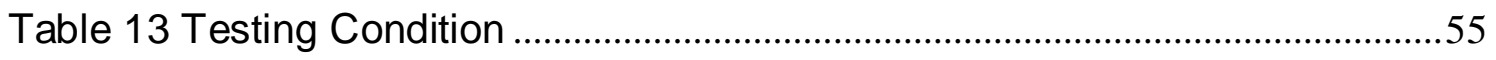

Table 14 Compare RMSE in different state ..........................................................59 


\section{Abstract}

The presents a Fault Tolerant and Data Fusion (FTDF) algorithm for a Ducted Fan Uninhabited Aerial Vehicle (DFUAV) Navigation System. The algorithm have two parts: the Gradient Descent (GD) for the Attitude and Heading Reference System (AHRS) and the Interacting Multiple Model (IMM) for position estimation. The GD methodology was designed is to fuse gyroscope, accelerometer, and geomagnetic sensor. The IMM algorithm is able to identify and compensate for multiple sensors data failures. There are three parts to this paper.

Frist, system identification technique are used and the Allan Variance method is used to build dynamic models and noise models for multiple Sensors and Actuators.

Second, a GD filter applicable to the Inertial Measurement Unit (IMU) consisting of tri-axis gyroscopes, accelerometers and magnetometers. The MARG (Magnetic, Angular Rate, and Gravity) implementation incorporates magnetic distortion and gyroscope bias drift compensation. The filter uses a quaternion representation, allowing accelerometer and magnetometer data to be used in an analytically derived and optimized gradient-descent algorithm to compute the direction of the gyroscope measurement error as a quaternion derivative.

Finally, The IMM algorithm is used to combine data from multiple sensors simultaneously. This filter uses multiple models that incorporate sensor failures. The probabilities of these models being correct is generated by the IMM. These probabilities can be used to identify sensor failures and compensate for these failures.

Keywords: Navigation System, Interacting Multiple Model, Gradient Descent, Fault Tolerant 


\section{Introduction}

Unmanned Aerial Vehicle (UAV) is one kind of robotic that are computerized and autonomous using a digital system as a pilot. Thus there is no risk of loss of life and the aircraft is easier to maintain than manned aircraft. Vertical Take-Off and Landing Unmanned Aerial Vehicle (VTOL) UAV operate in level flight without an airstrip, so they are widely used in surveillance and transportation. Examples are Aerie Long Endurance UAV and Eagle Eye'sTR-911 X VT-UAV shown in Figure 1.1.

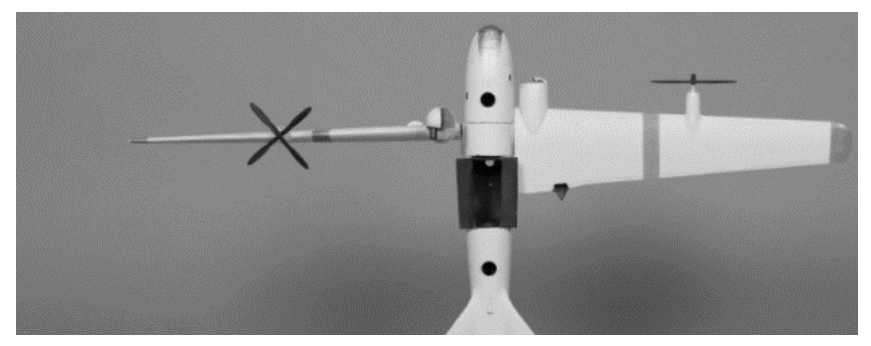

(a) Aerie Offers Long Endurance UAV

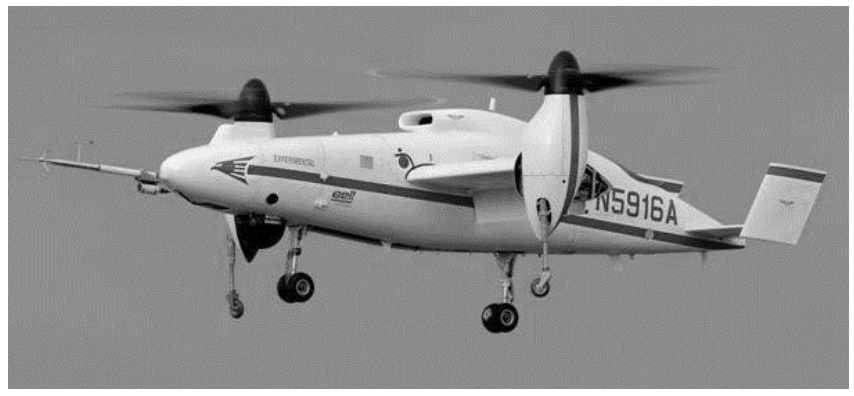

(b) Eagle Eye TR-911 X VT-UAV

Figure 1.1 VTOL UAV

Actually aircraft navigation system design process is similar with observer designing. Base on observer designing theory, several factor needs to Identify and know such as dynamic model of the aircraft, the sensor model and noise source model. Firstly, Dynamics model of the aircraft identify for building filter 
model and simulation. They are include engine thrust model, airfoil lift-drag model and ground effect model, these models are nonlinear, time-varying generally. Secondly, sensors model and noise source identify to get an accurate physical measuring. The high sensitivity sensors are susceptible from externally and internally noise, such as vibration noise, electrical noise, thermal noise and electromagnetic interference (EMI), so there are different noise distribution with measured values. If want to filter those noises, different methods are required to recognize the noise model and parameters.

In the UAV navigation system can be separate into two parts. A high sensitivity aircraft attitude measurement system, which belonging to the lower information fusion, requires high update frequency, high sensitivity. A high accuracy position measurement system which achieve for multiple sensor data fusion, high accuracy and fault tolerance ability. Aircraft attitude estimate system is composed by strapdown inertial sensors, such as gyroscopes, accelerometers and geomagnetic etc. body motion information of UAV will detected by those sensor. An efficient attitude conversion algorithm to process attitude information of the aircraft into earth coordinate system, these data are almost nonlinear. Currently, some algorisms can handle multi-sensor data fusion problem. They are Extended Kalman Filter, and Gardens Descent Filter, etc. For position of the aircraft mensuration which belong to middle-level data fusion, the systems can fuse GPSs, barometric pressure sensor, laser radar, air system of aircraft and other measuring equipment data to generate velocity and position information. Particle Filtering, Unscented Kalman Filter, Federated Filter are availability. Position and speed data may are from different reference frame and update frequencies the filter should be able to unify into a space-time, the final output information are latitude, longitude and altitude in Earth coordinate. 
The VTOL UAV is a nonlinear, multivariable, close coupe problem. Multiple dynamic models can be constructed for the VTOL UAV to account for maneuvering and sensor failures. An Interacting multiple (IMM) filter is used for data fusion and fault detection, this paper presents two independent systems to process attitude and position information. The Attitude and Heading Reference System (AHRS) is a gradient descent algorithm. The Position Estimation System (PES) uses an IMM algorithm. These algorithm are shown in Figure 1.2 along with their inter connections.

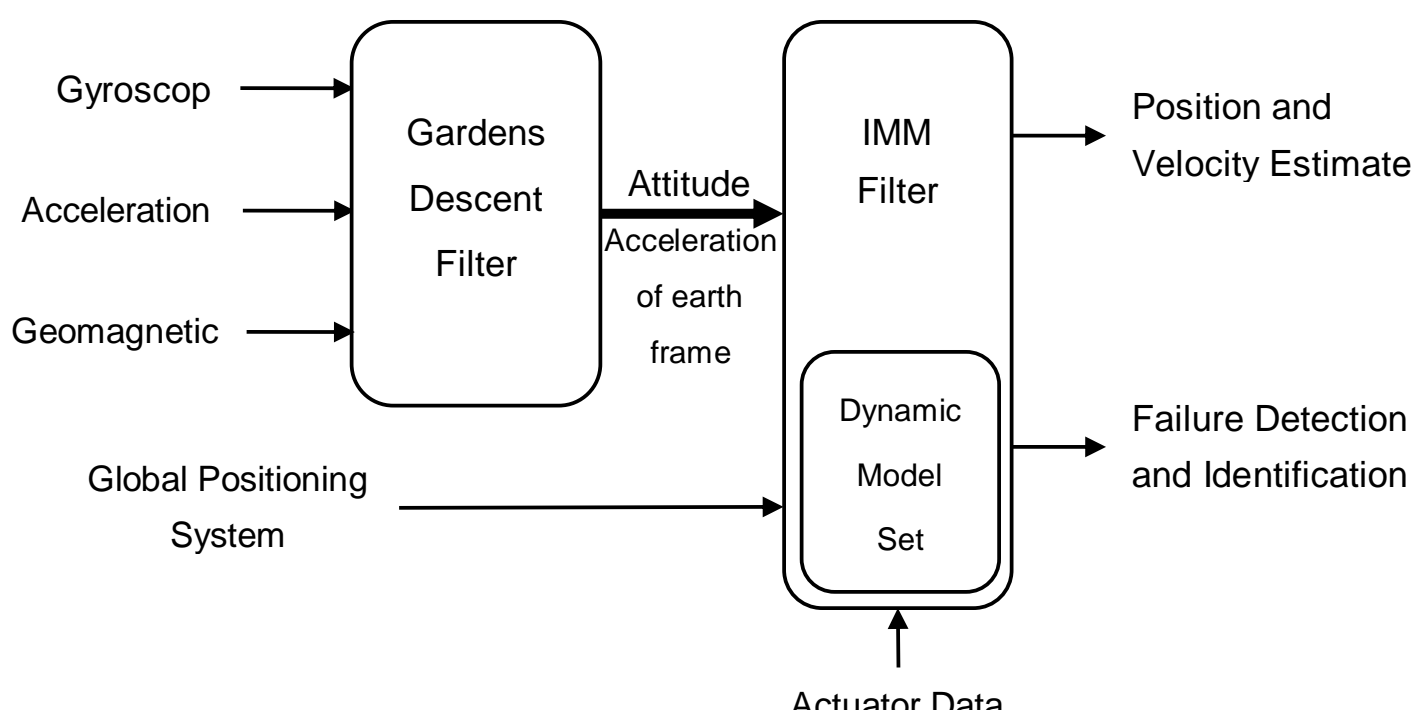

Figure 1.2 VTOL UAV Navigation System Connections

\subsection{How to Design a Data Fusion and Fault Tolerant System}

There are three technological key point for the Navigation system Designing.

First, establish mathematical models including kinetics models and the noise models, we can identify the model parameters by the methods of linear system identification and variance parameters of noise by statistical (Allan Variance). The presentation shown in chapter 2. 
Secondly, a gradient descent algorithm is used to fusion IMU's tri-axis gyroscopes, accelerometers and magnetometers. A motion detection algorithm to turn off/on the bias correction information form the gradient descent algorithm. The shown in chapter 3.

Finally, to build the interacting multiple model (IMM) filter for position estimation. It is an adaptive filter. It can automatically remove the lager error data on the system by the fault sensor state assessment. Which allows DFUAV under the situation of partial damage to the equipment remains normal flight. Chapter 4 is shown the detailed introduction.

\section{Modeling and Noise Identification}

\subsection{Coordinate Systems}

If build equations of motion of UAV to calculate the relative position, speed, acceleration and forces' vector, two axes' coordinates must be mentioned.

- Body-Axes Coordinate System Oxb yb zb

- $\quad$ Earth-Axes Coordinate System Oxg yg zg

a) Transfer between Oxg yg zg and Oxb yb zb

The attitude of UAV in the body frame transform to earth frame which are usually described by three Euler angles. They are yawing $(\psi)$, pitching $(\theta)$ and rolling $(\varphi)$ respectively. System. The transform function shown as:

$R_{e b}=\left[\begin{array}{ccc}\cos \theta \cos \psi & \sin \theta \sin \phi \cos \psi-\cos \phi \sin \psi & \sin \theta \cos \phi \cos \psi+\sin \phi \sin \psi \\ \cos \theta \sin \psi & \sin \psi \sin \theta \sin \phi+\cos \psi \cos \phi & \sin \theta \cos \phi \sin \psi-\sin \phi \cos \psi \\ -\sin \theta & \cos \theta \sin \phi & \cos \theta \cos \phi\end{array}\right]$ 
b) Estimate flat earth position from geodetic latitude, longitude, and altitude

For UAV position control is normal in a Cartesian Coordinate Plane. However the GPS use the WGS-84 World Geodetic System comprises a standard coordinate for the Earth. It output the latitude, longitude and altitude data, but it is not easy to For UAV Control, we need to use an algorithm transform The LLA a geodetic latitude $\mu$, longitude $l$, and altitude (h) into a Flat Earth position $(x, y, h)$. The flat Earth coordinate system assumes the $z$-axis is downward positive. The estimation begins by finding the small changes in latitude and longitude from the output latitude and longitude minus the initial latitude and longitude.

$$
\begin{aligned}
& d \mu=\mu-\mu_{0} \\
& d l=l-l_{0}
\end{aligned}
$$

where $d \mu$ is changes in latitude and $d l$ is changes in longitude. $\mu_{0}, l_{0}$ is origin coordinate in latitude and longitude.

$$
\begin{aligned}
& R_{N}=\frac{R}{\sqrt{1-\left(2 f-f^{2}\right) \sin ^{2} \mu_{0}}} \\
& R_{M}=R_{N} \frac{1-\left(2 f-f^{2}\right)}{1-\left(2 f-f^{2}\right) \sin ^{2} \mu_{0}}
\end{aligned}
$$

Where $(\mathrm{R})$ is the equatorial radius of the planet and ${ }^{f}$ is the flattening of the planet. In WGS84 $R=6378137.0 ; f=1 / 298.257223563$;

$$
\begin{aligned}
& \dot{N}=\frac{d \mu}{\arctan \left(\frac{1}{R_{M}}\right)} \\
& \dot{E}=\frac{d l}{\arctan \left(\frac{1}{R_{N} \cos \mu_{0}}\right)}
\end{aligned}
$$

where $\dot{N}$ is North small changes positions, $\dot{E}$ is East positions small changes.

With the conversion of the North and East coordinates to the flat Earth $\mathrm{x}$ and $\mathrm{y}$ coordinates, the transformation has the form of 
$\left[\begin{array}{l}p_{x} \\ p_{y}\end{array}\right]=\left[\begin{array}{cc}\cos \psi & \sin \psi \\ -\sin \psi & \cos \psi\end{array}\right]\left[\begin{array}{l}N \\ E\end{array}\right]$

where $^{(\psi)}$ is the angle in degrees clockwise between the $\mathrm{x}$-axis and north

$p_{z}=-h-h_{r e f}$

where $h_{\text {ref }}$ is the flat Earth z-axis value, it is the negative altitude minus.

\subsection{Noise Model of Sensors}

The normal sensor noises sources are scale factor errors (SFEs), drift, axis misalignments, angle random walk, bias Instability, rate random walk, and rate ramp noise to as shown in figure 3.2. Where $N$ is Angle Random walk, $B$ is Bias Instability, $K$ is Rate Random Walk, and $R$ is Rate Ramp Noise.

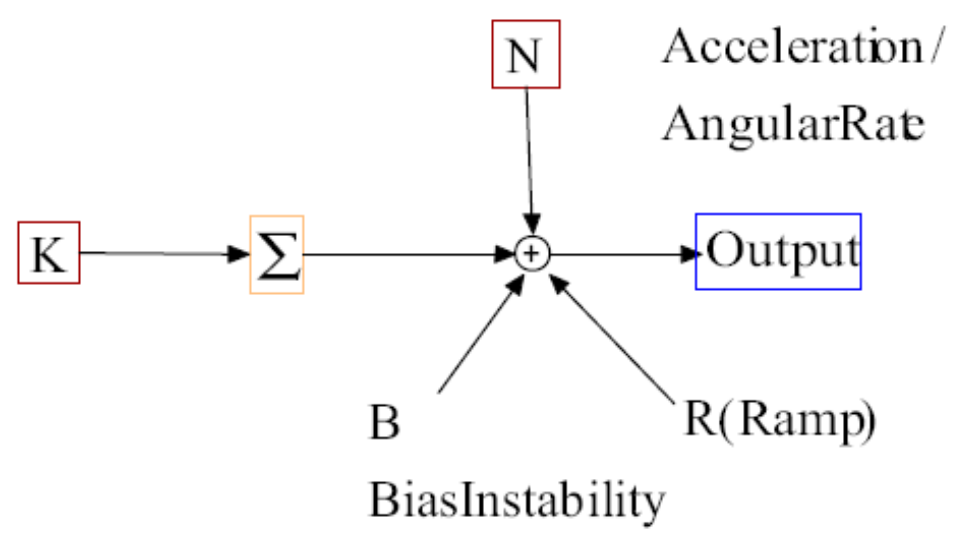

Figure 2.1 Normal Sensor Noises Model Structure

In multivariable output sensor, Have more character such as scale factor errors (SFEs), drift, input - axis misalignments, input/output nonlinearities.

Those can be describe by equation: 
$Y_{\text {out }}=S^{-1} \cdot(I+M) X_{\text {in }}+\vec{b}_{t}+\vec{w}_{w}$

$\vec{b}_{t}=\vec{b}_{f b}+\vec{b}_{r i}$

$\dot{\vec{b}}_{r i}=-\frac{1}{\tau} \vec{b}_{b s}+\vec{w}_{r w}$

where $\vec{b}_{t}$ Total of Bias; $\vec{b}_{f b}$ Fix of Bias; $\vec{b}_{r i}$ Bias Instability; $\vec{w}_{w}$ white noise;

$S$ Scale Factor; $M$ Misalignment;

\subsection{Root Mean Square Error (RMSE)}

The RMSE of an estimator $x$ with respect to an estimated parameter $x^{j}$ is defined as the square root of the mean square error:

$$
\operatorname{RMSE}(k)=\sqrt{\frac{1}{M} \sum_{j=1}^{M}\left(x(k)-x^{j}(k)\right)\left(x(k)-x^{j}(k)\right)^{T}}
$$

\subsection{Measurements for Multiple Sensors}

a) Inertial Measurement Unit

IMU that combines three Gyroscopes, a 3-axis Accelerometer, a 3-axis Geomagnetic Sensor, and a Barometric Pressure Sensor package given in Fig. 3.

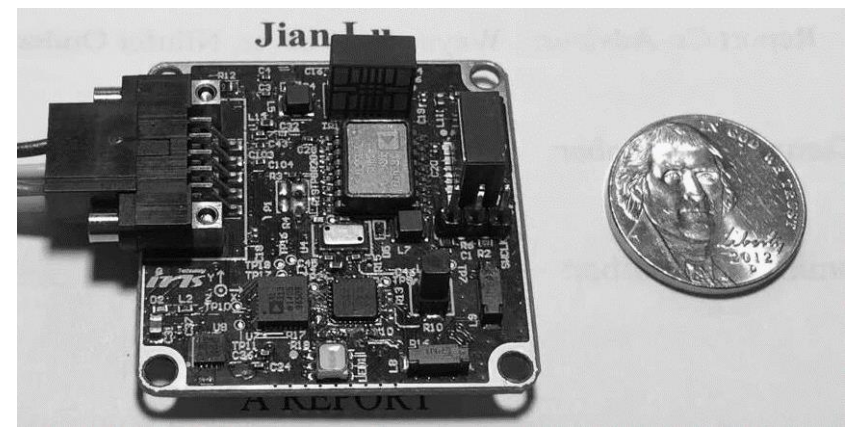

Figure 2.2 Inertial Measurement Unit

- Three High Vibration Immunity Digital Gyroscopes (ADXRS450) 
$\pm 300 \%$ sec angular rate sensing ${ }^{[10]}$;

Sensitivity to Linear Acceleration $0.03 \% \mathrm{sec} / \mathrm{g}$;

Excellent $25^{\circ}$ /hour null offset stability

The gyroscope noise model is giving in equation:

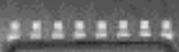

Dineriog

$\left[\begin{array}{c}\omega_{m x} \\ \omega_{m y} \\ \omega_{m z}\end{array}\right]=\left[\begin{array}{ccc}\frac{1}{S_{x}} & M_{x y} & M_{x z} \\ M_{y x} & \frac{1}{S_{y}} & M_{y z} \\ M_{z x} & M_{z y} & \frac{1}{S_{z}}\end{array}\right]\left[\begin{array}{l}\omega_{x} \\ \omega_{y} \\ \omega_{z}\end{array}\right]+G f_{g}^{b}+\vec{b}_{g}+\vec{w}_{g}$

$\vec{b}_{g}=\vec{b}_{f b}+\vec{b}_{r i}, \quad \dot{\vec{b}}_{r i}=-\frac{1}{\tau} \vec{b}_{b s}+\vec{w}_{r w}$

where $\omega_{m x}, \omega_{m y}, \omega_{m z}$ is the measured angular velocity;

$\omega_{x}, \omega_{y}, \omega_{z}$ represents the true angular velocity in the body frame;

$G$ is a $3 \times 3$ matrix encompassing the acceleration-sensitivity coefficients;

$S$ is the scale factor;

$M$ is the misalignment;

$\vec{b}_{g}$ is the gyroscope total of bias;

$\vec{b}_{r i}$ is the gyroscope bias Instability;

$\vec{w}_{g}$ is the gyroscope white noise in rate;

$\vec{w}_{r w}$ is the gyroscope white noise in bias random walk;

- 3-axis Digital Accelerometer (ADXL313)

Resolution as $0.025^{\circ}$ of inclination [8]

Low noise performance

$150 \mu \mathrm{g} / \sqrt{ } \mathrm{Hz}$ typical for $\mathrm{X}$ - and $\mathrm{Y}$-axes

$250 \mu \mathrm{g} / \sqrt{\mathrm{Hz}}$ typical for the Z-axis

The accelerometer noise model is giving in equation: 
$\left[\begin{array}{l}a_{m x} \\ a_{m y} \\ a_{m z}\end{array}\right]=\left[\begin{array}{ccc}\frac{1}{S_{x}} & M_{x y} & M_{x z} \\ M_{y x} & \frac{1}{S_{y}} & M_{y z} \\ M_{z x} & M_{z y} & \frac{1}{S_{z}}\end{array}\right]\left(R_{\theta, \varphi}\left[\begin{array}{l}a_{x} \\ a_{y} \\ a_{z}\end{array}\right]+\left[\begin{array}{ccc}0 & \omega_{y}^{2} & \omega_{z}^{2} \\ \omega_{x}^{2} & 0 & \omega_{z}^{2} \\ \omega_{x}^{2} & \omega_{y}^{2} & 0\end{array}\right]\left[\begin{array}{l}\Delta x \\ \Delta y \\ \Delta z\end{array}\right]+\vec{b}_{a}+\vec{w}_{a}\right)$

$a_{m x}, a_{m y}, a_{m z}$ is the measured acceleration

$a_{x}, a_{y}, a_{z}$ represents the true acceleration in the body frame

where $\Delta x, \Delta y, \Delta z$ is error which is a distance from the "center" of the Inertial Measurement Unit (IMU) to center-of-gravity position.

$S$ is the scale factor;

$M$ is the misalignment;

$\omega_{y}, \omega_{x}, \omega_{z}$ is the angular velocity of three axis.

$\vec{b}_{a}$ is the acceleration bias;

$\vec{w}_{a}$ is the acceleration white noise in rate.

$R_{\theta, \varphi}$ is the rotation angle for the tilt effect.

- 3-axis Geomagnetic Sensor (RM3100)

It over 10 times better resolution and over 20 times lower noise than the leading Hall Effect sensor ${ }^{[6]}$.

Geomagnetic noise model, Induced effect of the soft iron and hard iron.

$\left[\begin{array}{c}m_{m x} \\ m_{m y} \\ m_{m z}\end{array}\right]=\left[\begin{array}{ccc}\frac{1}{S_{x}} & M_{x y} & M_{x z} \\ M_{y x} & \frac{1}{S_{y}} & M_{y z} \\ M_{z x} & M_{z y} & \frac{1}{S_{z}}\end{array}\right]\left(R_{\theta, \varphi}\left[\begin{array}{l}m_{x} \\ m_{y} \\ m_{z}\end{array}\right]+\vec{b}_{a}+\vec{w}_{a}\right)$

where $m_{m x}, m_{m y}, m_{m z}$ represents the measured magnetic field; 
$m_{y}, m_{x}, m_{z}$ represents the true measured magnetic field in the body frame;

$S$ is the Scale Factor;

$M$ is the misalignment Induced effect of soft iron;

$\vec{b}_{a}$ is the bias Induced effect of hard iron;

$\vec{w}_{a}$ is the white noise in rate;

$R_{\theta, \varphi}$ is the rotation angle for the tilt effect;

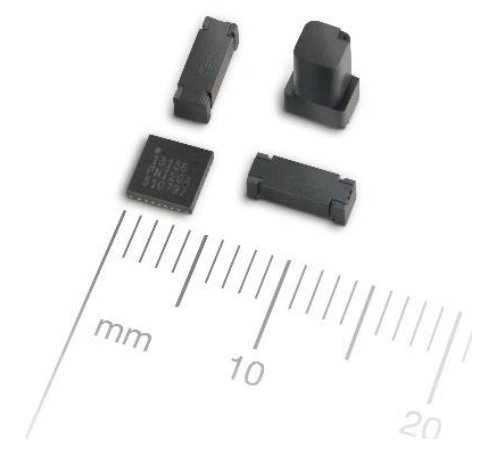

\section{- Barometric Pressure Sensor (MS5611-01BA03)}

High resolution module, $10 \mathrm{~cm}$

Fast conversion down to $1 \mathrm{~ms}$

Operating range: 10 to $1200 \mathrm{mbar},-40$ to $+85^{\circ} \mathrm{C}$

Barometric Pressure Sensor noise model

$h_{m}=S \cdot h+\vec{b}_{h}+\vec{w}_{h}$

$\vec{b}_{h}=\vec{b}_{f h}+\vec{b}_{r i}, \quad \dot{\vec{b}}_{r i}=-\frac{1}{\tau} \vec{b}_{h s}+\vec{w}_{r w} ;$

where $h_{m}$ is the represents the measured high;

$h$ is the represents the true high;

$S$ is the scale factor;

$M$ is the misalignment Induced effect of soft iron;

$\vec{b}_{h}$ is the Barometric Pressure sensor long run bias;

$\vec{w}_{h}$ is the white noise in rate. 


\section{- GPS Module}

The GPS Module is the newest family of standalone GPS/GNSS modules from u-blox. With the exceptional performance of the $\mathrm{u}$ blox 7 multi-GNSS (GPS, GLONASS, Galileo, QZSS and SBAS) engine. The GPS Module provides maximum sensitivity while maintaining low system power.

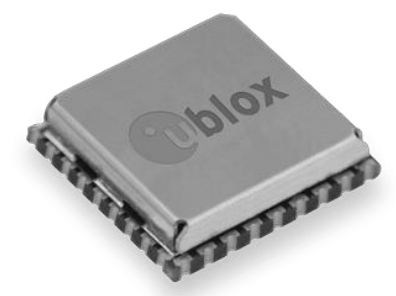

Table 1 Specification of GPS ${ }^{[11]}$

\begin{tabular}{|l|l|}
\hline Receiver type & $\begin{array}{l}\text { 56-channel } \\
\text { GPS/QZSS L1 C/A, GLONASS L1 FDMA, } \\
\text { SBAS: WAAS, EGNOS, MSAS }\end{array}$ \\
\hline Navigation update rate up to & $10 \mathrm{~Hz}$ \\
\hline Accuracy GPS / GLONASS & $\begin{array}{l}\text { Position } 2.5 \mathrm{~m} \text { CEP / } 4.0 \mathrm{~m} \\
\text { SBAS } 2.0 \mathrm{~m} \text { CEP / n.a. }\end{array}$ \\
\hline Acquisition GPS / GLONASS & $\begin{array}{l}\text { Cold starts: } 29 \mathrm{~s} / 30 \mathrm{~s} \\
\text { Aided starts: } 5 \mathrm{~s} / \mathrm{n} \cdot \mathrm{a} . \\
\text { Reaquisition: } 1 \mathrm{~s} / 3 \mathrm{~s}\end{array}$ \\
\hline Sensitivity GPS / GLONASS & $\begin{array}{l}\text { Tracking: }-162 \mathrm{dBm} /-158 \mathrm{dBm} \\
\text { Cold starts: }-148 \mathrm{dBm} /-140 \mathrm{dBm} \\
\text { Warm starts: }-148 \mathrm{dBm} /-145 \mathrm{dBm}\end{array}$ \\
\hline
\end{tabular}

GPS positioning is a basic principle of triangulation, using satellite GPS receivers to measure the radio signal transmission delay time to calculate the distance. Three or more distances and the position of the satellite can be used to determine the GPS receiver's position. GPS accuracy's deserted by a lot of factors such as lonospheric effects, Shifts in the satellite orbits, clock errors of the satellites' clocks, multipath effects, tropospheric effects and calculation rounding errors.

The errors of the GPS system are summarized in the following table 2. The individual values are not constant values, but are probability. All numbers are approximate values.

Table 2 Errors of the GPS System [2]

\begin{tabular}{|l|l|}
\hline \multicolumn{1}{|c|}{ Sources of Errors in GPS } & \multicolumn{1}{c|}{ Standard deviation } \\
\hline Ionospheric effects & \pm 5 meters \\
\hline Shifts in the satellite orbits & \pm 2.5 meter \\
\hline
\end{tabular}




\begin{tabular}{|l|l|}
\hline Clock errors of the satellites' clocks & \pm 2 meter \\
\hline Multipath effect & \pm 1 meter \\
\hline Tropospheric effects & \pm 0.5 meter \\
\hline Calculation- und rounding errors & \pm 1 meter \\
\hline
\end{tabular}

The GPS noise equation can desecration into the Pseudo-range and time error estimation equation.

Pseudo-range and time error equation:

$$
\begin{aligned}
\tau & =T b-T a \\
& =(\tau b-\Delta t s)-(\tau a-\Delta t r) \\
& =(\tau b-\tau a)+\Delta t r-\Delta t s \\
\tau & =(\tau b-\tau a)+\Delta t r-\Delta t s+\delta_{t} \\
c \tau & =c[(\tau b-\tau a)+\Delta t r-\Delta t s+\Delta t a] \\
P R_{i} & =R_{i}+c \delta_{t} \\
\delta_{t} & =\Delta t r-\Delta t s+\Delta t a \\
\mathrm{R} & =\sqrt{\left(X-X_{i}\right)^{2}+\left(Y-Y_{i}\right)^{2}+\left(Z-Z_{i}\right)^{2}} \\
P R_{i} & =\sqrt{\left(X-X_{i}\right)^{2}+\left(Y-Y_{i}\right)^{2}+\left(Z-Z_{i}\right)^{2}}+c \delta_{t}+w_{i}(i=1,2,3,4)
\end{aligned}
$$

$P R_{i}$ Pseudo-range is for GPS;

$X_{i}, Y_{i}, Z_{i}$ are the GPS satellites position is in Earth-centered inertial (ECl) coordinate frames.

$X, Y, Z$ are the Receiver position is in $\mathrm{ECl}$. (Unknown)

$\delta_{t}$ is the clock error. (Unknown)

$w_{i}$ is the total of noise 
DOP (Dilution of precision)

DOP descriptor error of estimate with sources of errors in GPS in dynamic condition. Those data can tell us the GPS precision situation in real time. [3]

There are six kind of DOP:

- GDOP: Geometrical Dilution of Precision, (measure of accuracy in 3-D position and time)

- $\mathrm{GDOP}^{2}=\mathrm{PDOP}^{2}+\mathrm{TDOP}^{2}$

- TDOP: Time Dilution of Precision (measure of accuracy in Time)

- PDOP: Position Dilution of Precision (measure of accuracy in 3-D position), also called spherical DOP

- $\quad$ PDOP $^{2}=$ HDOP $^{2}+V_{D O P} 2$

- HDOP: Horizontal Dilution of Precision (measure of accuracy in 2-D position, for example Latitude and Longitude)

- VDOP: Vertical Dilution of Precision (measure of accuracy in 1-D position, Height)

We do a test which receive GPS position and velocity data in ECEF (Earth-Centered, EarthFixed) coordinate for $10 \mathrm{~min}$ at $5 \mathrm{~Hz}$ sampling frequency. The GPS data Variance statistics show in Figure 2.
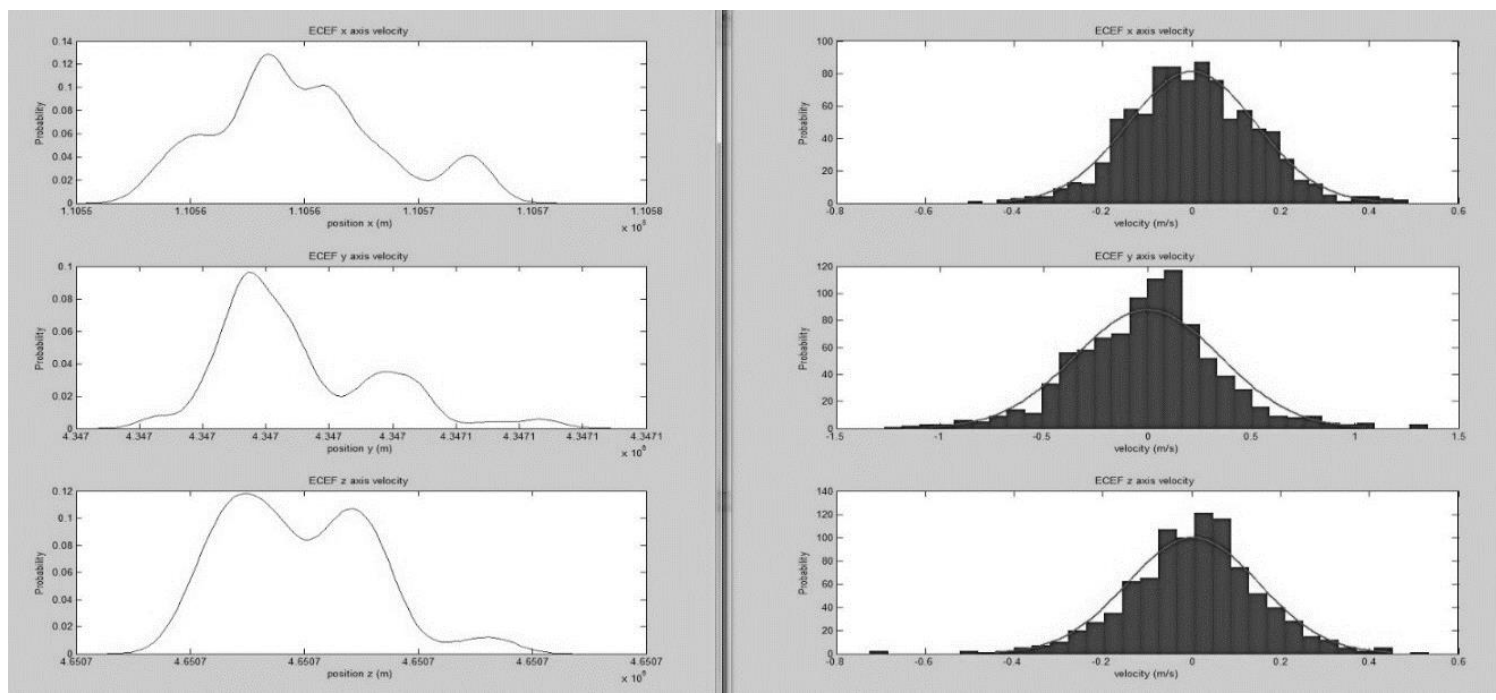

Figure 2.3 GPS Position and Velocity Variance 
GPS Position signal with color noise, but velocity approximate a white noise. Because the UAV power support limit, the flight time only $10 \mathrm{~min}$ at VTOL state. The GPS noise model can be consider a first-order Markov model.

$p_{x, y, z}=\hat{p}_{x, y, z}+\vec{b}_{r i}+\vec{w}_{w}, \quad \dot{\vec{b}}_{r i}=-\frac{1}{\tau} \vec{b}_{b s}+\vec{w}_{r w}$

$\vec{w}_{w}$ Gaussian noise;

$\vec{w}_{r w}$ Gaussian noise for Bias random walk;

$p_{x, y, z}$ Position data with noise and bias;

$\hat{p}_{x, y, z}$ Estimate position data without noise and bias;

\section{- Rotation Servomotor}

A robotics digital servo is state of the art modular smart servos incorporating motor, gear reducer, control circuitry and communications capability in one single package. Servo is capable of detecting and controlling angle position and rotation speed.

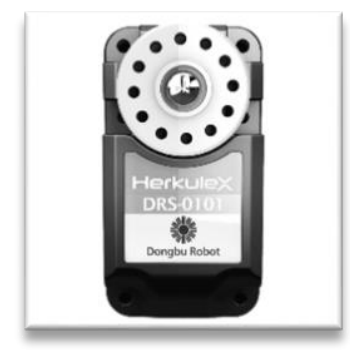

\section{- RPM Sensor for High-Voltage ESC}

It detects the voltage changes at the wires of brushless motor, and then outputs the RPM signal. This RPM sensor can work with some speed control systems for helicopters. RPM testing range (for 2 poles brushless motor): 1000rpm

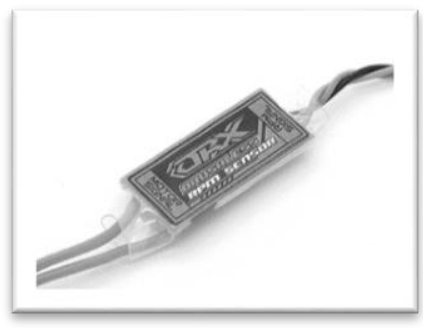
to $300000 \mathrm{rpm}$. 


\subsection{Noise Identify Technique}

Allan variance by the national bureau of standards, is put forward in 1960. It is a kind of time domain analysis technology which is recognized generally by the IEEE standard analysis method [2] of gyroscope performance. The characteristics of this method is easy to compare it to various error sources of a detailed characterization and identification of statistical properties. Noise sources may exist within the instrument and even don't know the mechanical structure of the instrument, as long as the test system, can find the noise source. Allan variance can be used as a single data analysis method, also can be used to supplement the frequency domain analysis technology, this technology can be applied to any instrument noise research.

Allan variance equations can write that:

$$
\sigma_{y}^{2}\left(n \tau_{0}, N\right)=\operatorname{AVAR}\left(n \tau_{0}, N\right)=\frac{1}{2 n^{2} \tau_{0}^{2}(N-2 n)} \sum_{i=0}^{N-2 n-1}\left(x_{i+2 n}-2 x_{i+n}+x_{i}\right)^{2}
$$

We base on the equation to calculate the solution. The Figure2.5 show the Gyro Allan variance statistics characteristic curve for 20,000 Second.

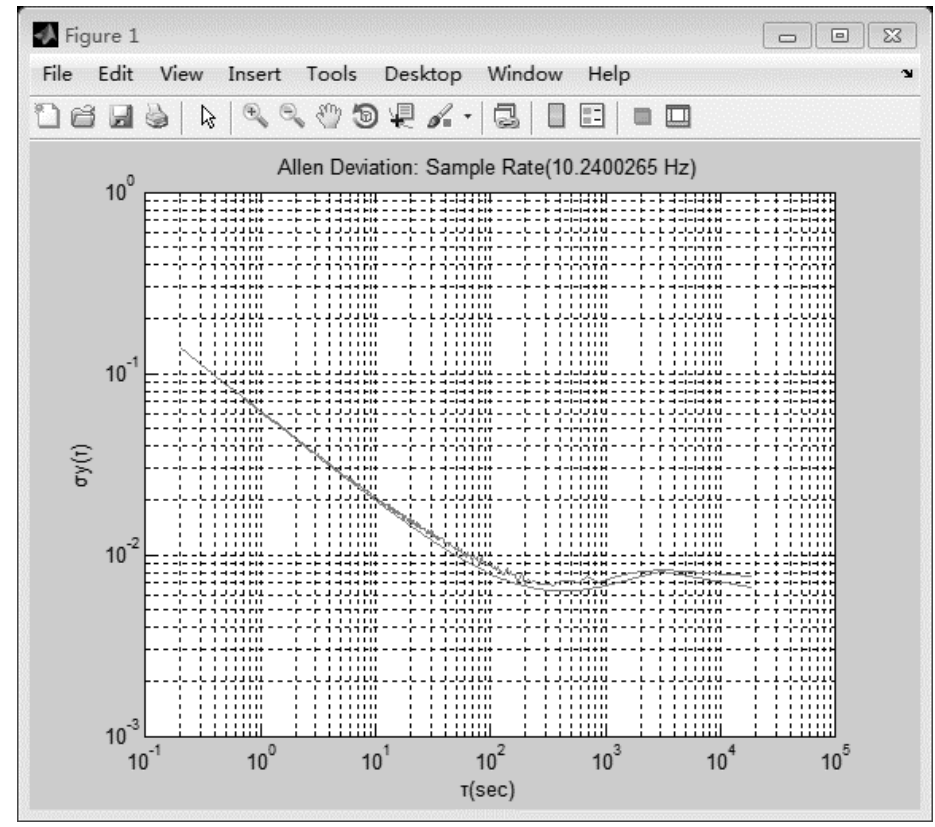

Figure 2.4 20,000 Sec. Gyroscope Allan Variance Statistics Characteristic 


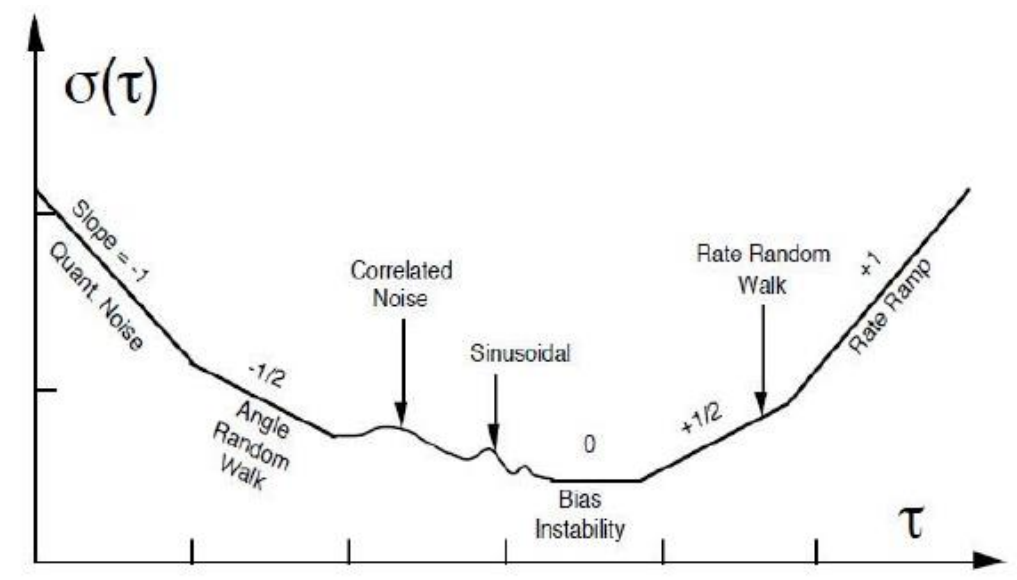

Figure 2.5 Typical Allan Deviation plot for a system ${ }^{[7]}$

The Noise Coefficient can be calculate from the table 3 .

Table 3 Allan Variance Parameters [3]

\begin{tabular}{|c|c|c|c|}
\hline Error Type & $\operatorname{PSD}\left(S_{\Omega}\right)$ & $\operatorname{AV}\left(\sigma^{2}(\tau)\right)$ & Comments \\
\hline $\begin{array}{c}\text { Quantisation } \\
\text { Noise }\end{array}$ & $(2 \pi f)^{2} Q^{2} T_{s}$ & $\frac{3 Q^{2}}{\tau^{2}}$ & $\begin{array}{l}\text { Q: Quant. } \\
\text { noise } \\
\text { coefficient }\end{array}$ \\
\hline $\begin{array}{l}\text { Random } \\
\text { Walk }\end{array}$ & $N^{2}$ & $\frac{N^{2}}{\tau}$ & $\begin{array}{l}\text { N: Random } \\
\text { walk } \\
\text { coefficient } \\
\end{array}$ \\
\hline $\begin{array}{c}\text { Bias } \\
\text { Instability }\end{array}$ & $\begin{array}{l}\left(\frac{B^{2}}{2 \pi}\right) \cdot \frac{1}{f} \\
f \leq f_{0}\end{array}$ & $\frac{2 B^{2}}{\pi}\left[\begin{array}{l}\ln 2- \\
\frac{\sin ^{3} x}{2 x^{2}}(\sin x+4 x \cos x) \\
+C i(2 x)-C i(4 x)\end{array}-\right.$ & $\begin{array}{l}\text { B: Bias } \\
\text { instability } \\
\text { coefficient. } \\
x: \pi f_{0} \tau \\
\mathrm{Ci}: \text { Cosine } \\
\text { integration } \\
\text { function }\end{array}$ \\
\hline $\begin{array}{l}\text { Rate } \\
\text { random } \\
\text { walk }\end{array}$ & $\left(\frac{K}{2 \pi}\right)^{2} \frac{1}{f^{2}}$ & $\frac{K^{2} \tau}{3}$ & $\begin{array}{c}\text { K: Rate } \\
\text { random walk } \\
\text { coefficient }\end{array}$ \\
\hline Rate ramp & $\frac{R^{2}}{(2 \pi f)^{3}}$ & $\frac{R^{2} \tau^{2}}{2}$ & $\begin{array}{l}\text { R: Rate ramp } \\
\text { coefficient }\end{array}$ \\
\hline $\begin{array}{c}\text { Correlated } \\
\text { Noise }\end{array}$ & $\frac{\left(q_{c} T_{c}\right)^{2}}{1+\left(2 \pi f T_{c}\right)^{2}}$ & $\begin{array}{l}\frac{\left(q_{c} T_{c}\right)^{2}}{\tau}, \tau \gg T_{c} \\
\frac{q_{c}^{2}}{3} \tau, \tau \ll T_{c}\end{array}$ & $\begin{array}{c}q_{c}: \text { Noise } \\
\text { amplitude } \\
T_{c}: \text { Correlation } \\
\text { time }\end{array}$ \\
\hline $\begin{array}{c}\text { Sinusoidal } \\
\text { noise }\end{array}$ & $\frac{\Omega_{0}^{2}}{2}\left[\begin{array}{c}\delta\left(f-f_{0}\right) \\
+\delta\left(f+f_{0}\right)\end{array}\right]$ & $\Omega_{0}{ }^{2}\left(\frac{\sin ^{2} \pi f_{0} \tau}{\pi f_{0} \tau}\right)^{2}$ & $\begin{array}{c}\Omega_{0} \text { :point } \\
\text { Amplitude } \\
f_{0} \text { :point } \\
\text { frequency } \\
\delta(x) \text { :Delta } \\
\text { function }\end{array}$ \\
\hline
\end{tabular}


The gyroscope root and Accelerometer root Allan variance show in Figure 2.6 and 2.7 from IMU Datasheet.

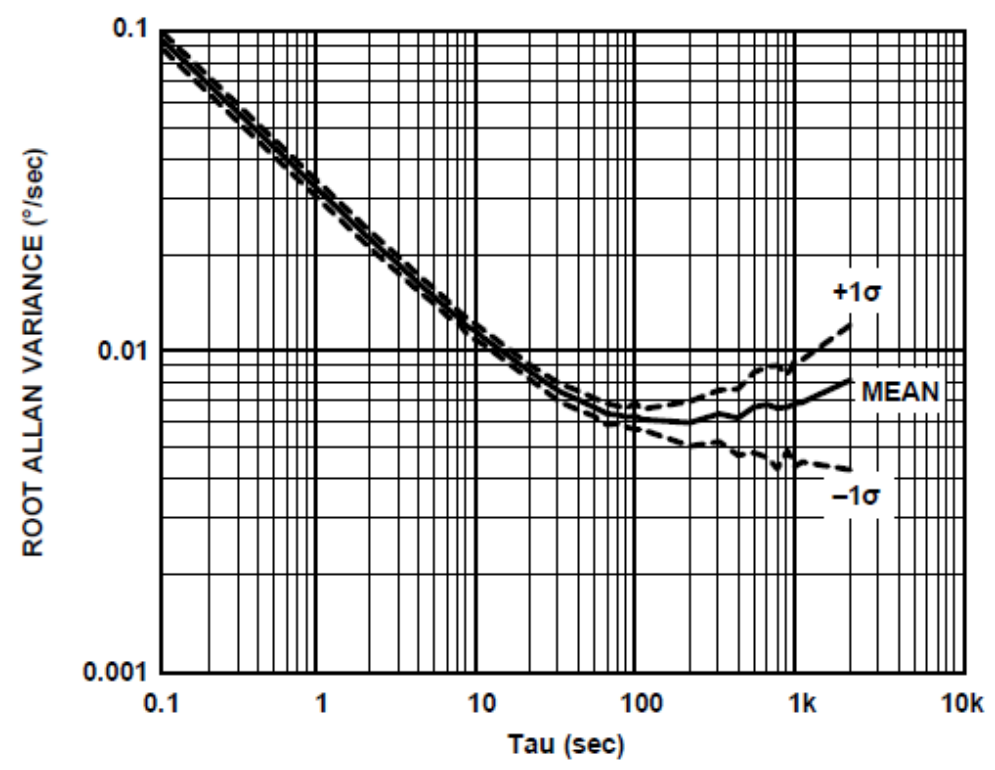

Figure 2.6 Gyroscope Root Allan Variance

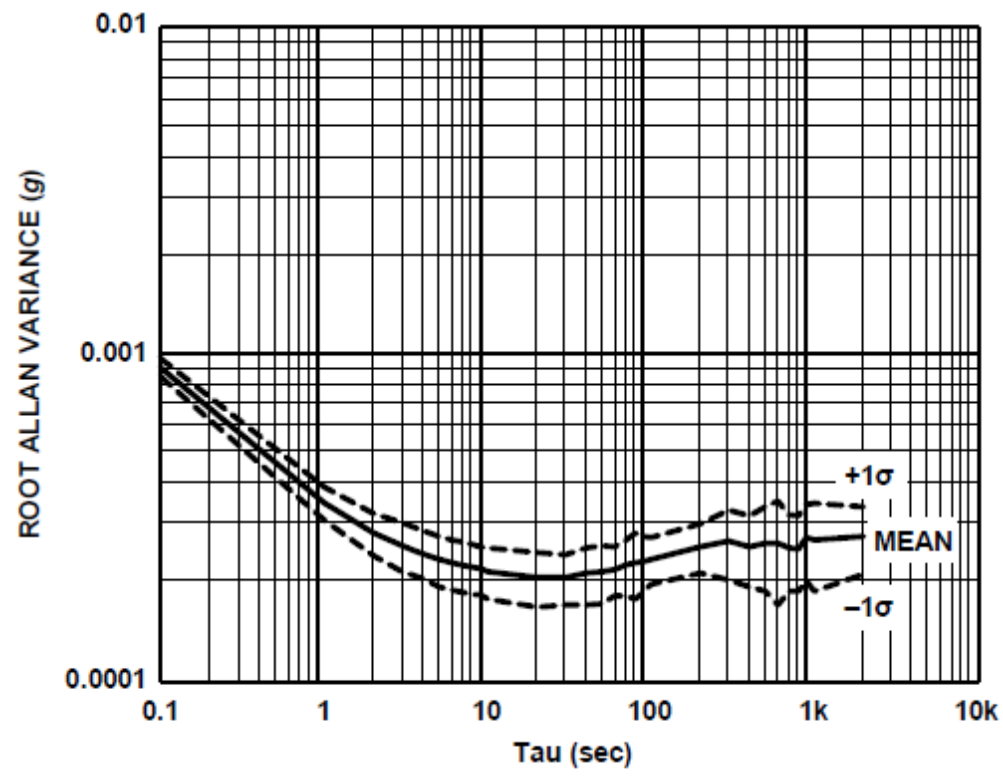

Figure 2.7 Accelerometer Root Allan Variance 


\subsection{Electronic Duct Fan Model}

Electronic Duct Fan system is a very imported part which support the power when the UAV run. Its characteristic influent the UAV stability and sensitivity dirtily, species in DFUAV State. The EDF system include Electronic Duct Fan, Brushless Electronic speed controller and PRM Sensor. As show in Figure2.1 Assembly of Electronic Duct Fan system.

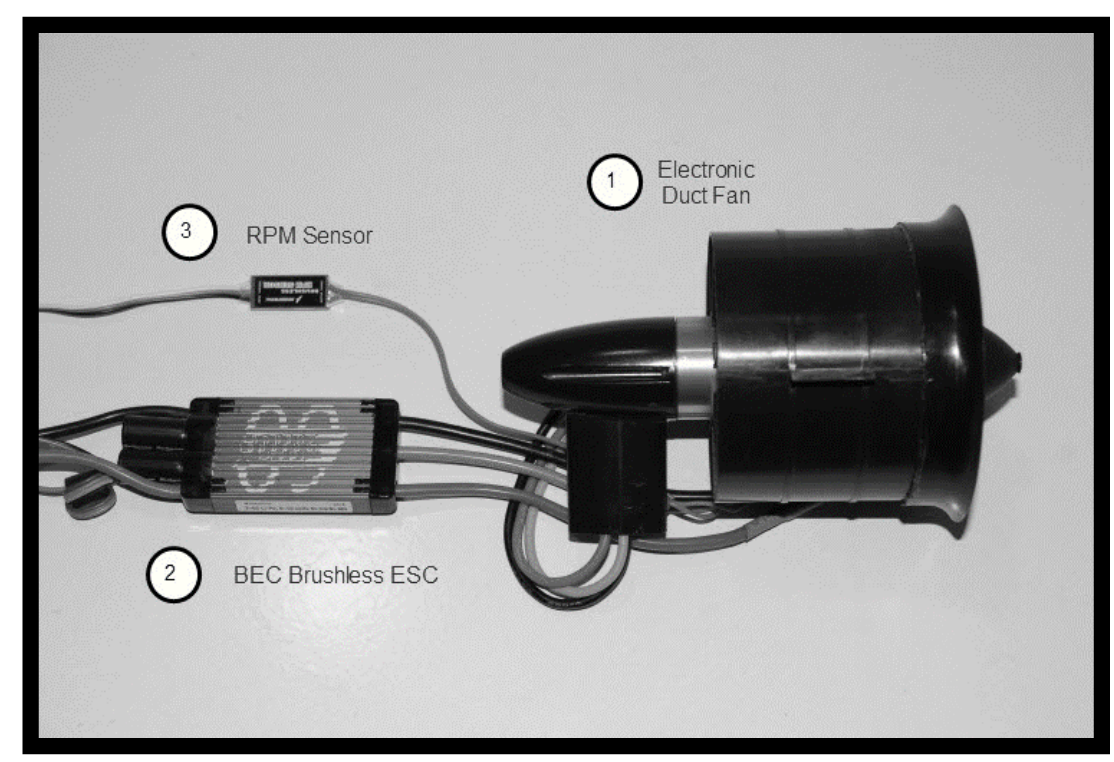

Figure 2.8 Assembly of Electronic Duct Fan system

The UAV main engine are two 12 Blade 70mm diameter Electronic Duct Fan.

The Specification of Electronic Duct Fan show in Table 4

Table 4 Specification of Electronic Duct Fan

\begin{tabular}{|l|l|}
\hline & 12 Blade $70 \mathrm{~mm}$ Electronic Duct Fan \\
\hline Outside Diameter : & $71.8 \mathrm{~mm}$ \\
\hline Material : & Fiber Reinforced Nylon Rotor \\
\hline Rotor Diameter : & $68 \mathrm{~mm}, 12$ blades \\
\hline Max RPM : & $52000 \mathrm{RPM}$ \\
\hline Motor Shaft : & $3.17 \mathrm{~mm} \& 4.0 \mathrm{~mm}$ \\
\hline Working Voltage : & $14.8 \mathrm{v}-22.2 \mathrm{v}(4-6 \mathrm{~S})$ \\
\hline Max Power : & $1800 \mathrm{~W}$ \\
\hline Motors required : 28mm Inrunner Motor & $2200-3300 \mathrm{Kv}$ \\
\hline
\end{tabular}


Electronic Duct Fan setup a $3600 \mathrm{Kv} 28 \mathrm{~mm}$ diameter inrunner motor supprte the power. The Specification of Brushless Motor show in Table 5

Table 5 Specification of Brushless Motor

\begin{tabular}{|l|l|}
\hline Type: & 6-pole Inrunner Brushless \\
\hline Size: & 15 -size for Ducted Fans \\
\hline Bearings or Bushings: & Two 4 x 10 x 4mm Bearings \\
\hline Voltage: & $11.1-16.8 \mathrm{~V}$ \\
\hline RPM/Volt (Kv): & 3600 \\
\hline Resistance (Ri): & 0.02 ohms \\
\hline Idle Current (lo): & $2.80 \mathrm{~A} @ 10 \mathrm{~V}$ \\
\hline Continuous Current: & $46 \mathrm{~A}$ \\
\hline Maximum Burst Current: & $55 \mathrm{~A} \mathrm{(15} \mathrm{sec)}$ \\
\hline Cells: & $3 \mathrm{~S}-4 \mathrm{~S} \mathrm{LiPo} \mathrm{power} \mathrm{10-14} \mathrm{Ni-MH/Ni-Cd} \mathrm{battery}$ \\
\hline Speed Control: & $60 \mathrm{~A} \mathrm{brushless}$ \\
\hline Weight: & $106 \mathrm{~g} \mathrm{(3.7} \mathrm{oz)}$ \\
\hline Overall Diameter: & $28 \mathrm{~mm}(1.10 \mathrm{in})$ \\
\hline Shaft Diameter: & $4 \mathrm{~mm}(0.16 \mathrm{in})$ \\
\hline Overall Length: & $40 \mathrm{~mm}(1.56 \mathrm{in})$ \\
\hline
\end{tabular}

In order to test the characteristics of the engine we designed a test system, as shown, the system includes a power sensor, voltmeter, power meter, tension meter, torque meter and anemometer. Can measure the Force, voltage, Power (W), Ampere, RPM, and Airspeed.

System identification is generally divided into two kinds of static and dynamic parameters. Static parameters mainly on account of the proportion of the characteristics of the various components in the static. We control EDF into different rotation speed then recode the data curve, we can very easily identify physical parameter equation system, such as to determine the order of the system. DC motor dynamic parameters considering various factors, including of Coulomb Friction Coefficient, Viscous Friction Coefficient, moment of inertia. Our data sampled at different engine speeds, as Table 6 Test Data of EDF

Table 6 Test Data of EDF

\begin{tabular}{|c|c|c|c|c|c|c|c|c|}
\hline Force(N) & $\begin{array}{c}\mathrm{V} \\
\text { power }\end{array}$ & Power(W) & A & RPM & PWM(ms) & Effect(g/W) & Airspeed(m/s) & K \\
\hline 0 & 14.96 & 1.9 & 0.13 & 0 & 0 & 0 & 0 & \\
\hline 0.784 & 14.92 & 28 & 1.9 & 7920 & 1.21 & 2.857142857 & 12.99139605 & 0.00164033 \\
\hline
\end{tabular}




\begin{tabular}{|c|c|c|c|c|c|c|c|c|}
\hline 1.274 & 14.88 & 51 & 3.34 & 10000 & 1.23 & 2.549019608 & 16.93870807 & 0.00169387 \\
\hline 2.744 & 14.77 & 119.2 & 8.15 & 14260 & 1.25 & 2.348993289 & 23.95495069 & 0.00167987 \\
\hline 3.528 & 14.69 & 163.3 & 11.14 & 15940 & 1.326 & 2.204531537 & 26.62443914 & 0.00167029 \\
\hline 4.41 & 14.58 & 223.4 & 15.23 & 17720 & 1.36 & 2.014324082 & 29.62494103 & 0.00167184 \\
\hline 5.096 & 14.48 & 265.2 & 18.54 & 18920 & 1.39 & 1.960784314 & 32.8659212 & 0.0017371 \\
\hline 5.88 & 14.34 & 330 & 22.98 & 20400 & 1.43 & 1.818181818 & 34.37200313 & 0.0016849 \\
\hline 6.566 & 14.62 & 381 & 26.56 & 21520 & 1.468 & 1.758530184 & 36.28299459 & 0.00168601 \\
\hline 7.252 & 14.05 & 443 & 31.8 & 22500 & 1.51 & 1.670428894 & 37.8761027 & 0.00168338 \\
\hline 8.232 & 13.89 & 515 & 37.18 & 24000 & 1.55 & 1.631067961 & 40.04217186 & 0.00166842 \\
\hline
\end{tabular}

\section{RPM and Thrust}

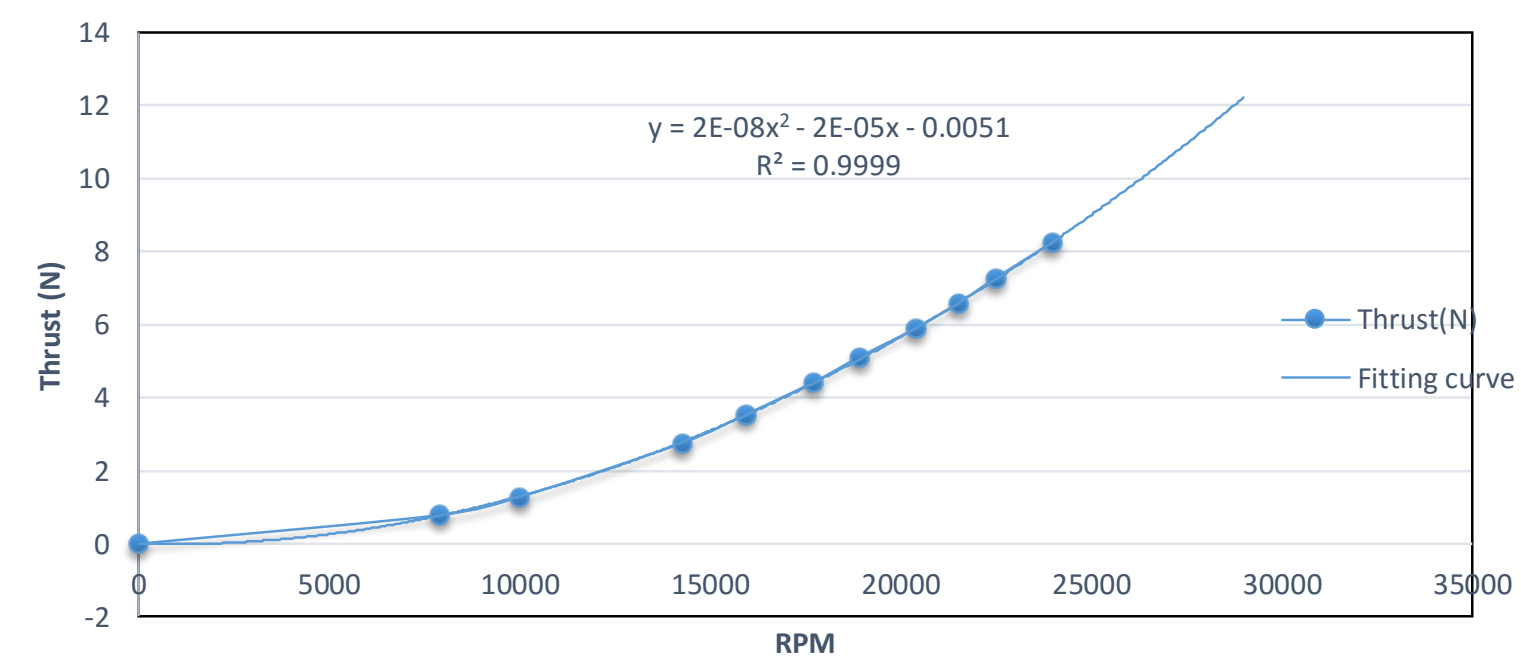

Figure 2.9 RPM and Thrust

The table shows us information from the chart, we can make a polynomial curve fitting original, and then a formula can calculated by MATLAB:

$$
y_{\text {thrust }}=(1.52938 e-08) \cdot x_{r p m}{ }^{2}-(2.2741 e-05) \cdot x_{r p m}-0.00511756
$$

where $y_{\text {thrust }}$ is thrust of EDF, $x_{r p m}$ is EDF rotate speed of propeller 


\subsection{CA and TCA Model}

At radar multiple target maneuvering tracking applications, Three kind filter models have been selected in common use. These models are a constant velocity (CV), a constant acceleration (CA), and a three-dimensional turn with a kinematic constraint (TURN), the turn value is estimation. In the AHRS, the threedimensional turn information can calculate form the INS, so the acceleration value can transform from body frame to the earth frame. The CV and CA model will use a rotation matrix to finish turn information fusion. The model will become a three-dimensional turn with a kinematic constraint (TCV) and a threedimensional turn with a dynamics constraint (TCA). A normal model show in below formula

$$
\begin{aligned}
& x(k+1)=A x(k)+B u(k)+G w(k) \\
& y(k)=C x(k)+H v(k)
\end{aligned}
$$

- CA Model

The state vector for the CA continuous time model is defined as

$$
\begin{aligned}
X & =\left[\begin{array}{lllllllll}
x & y & z & v_{x} & v_{x} & v_{x} & a_{x} & a_{y} & a_{z}
\end{array}\right] \\
A & =\left[\begin{array}{llll}
\operatorname{diag}_{3 \times 3}(0) & \operatorname{diag}_{3 \times 3}(1) & \operatorname{diag}_{3 \times 3}(0) \\
\operatorname{diag}_{3 \times 3}(0) & \operatorname{diag}_{3 \times 3}(0) & \operatorname{diag}_{3 \times 3}(1) \\
\operatorname{diag}_{3 \times 3}(0) & \operatorname{diag}_{3 \times 3}(0) & \operatorname{diag}_{3 \times 3}(0)
\end{array}\right] \\
C & =\operatorname{diag}_{9 \times 9}(1)
\end{aligned}
$$

Discrete-time CA model

$$
\begin{aligned}
& X=\left[\begin{array}{lllllllll}
x & y & z & v_{x} & v_{x} & v_{x} & a_{x} & a_{y} & a_{z}
\end{array}\right] \\
& A=\left[\begin{array}{ccc}
\operatorname{diag}_{3 \times 3}(1) & \operatorname{diag}_{3 \times 3}(\Delta t) & \operatorname{diag}_{3 \times 3}\left(\frac{1}{2} \Delta t^{2}\right) \\
\operatorname{diag}_{3 \times 3}(0) & \operatorname{diag}_{3 \times 3}(1) & \operatorname{diag}_{3 \times 3}(\Delta t) \\
\operatorname{diag}_{3 \times 3}(0) & \operatorname{diag}_{3 \times 3}(0) & \operatorname{diag}_{3 \times 3}(1)
\end{array}\right] \\
& B=\left[\begin{array}{lll}
\frac{\Delta t^{3}}{6} & \frac{\Delta t^{2}}{2} & \Delta t
\end{array}\right]^{T}
\end{aligned}
$$


where $\Delta t$ is the difference of the measurement time System and measurement noise convenience matrices for each state, this matrix is given as

$$
Q=\sigma_{q}^{2}\left[\begin{array}{ccc}
\operatorname{diag}_{3 \times 3}\left(\frac{\Delta t^{5}}{5}\right) & \operatorname{diag}_{3 \times 3}\left(\frac{\Delta t^{4}}{4}\right) & \operatorname{diag}_{3 \times 3}\left(\frac{\Delta t^{3}}{3}\right) \\
\operatorname{diag}_{3 \times 3}\left(\frac{\Delta t^{4}}{4}\right) & \operatorname{diag}_{3 \times 3}\left(\frac{\Delta t^{3}}{3}\right) & \operatorname{diag}_{3 \times 3}\left(\frac{\Delta t^{2}}{2}\right) \\
\operatorname{diag}_{3 \times 3}\left(\frac{\Delta t^{3}}{3}\right) & \operatorname{diag}_{3 \times 3}\left(\frac{\Delta t^{2}}{2}\right) & \operatorname{diag}_{3 \times 3}(\Delta t)
\end{array}\right], \quad R=\sigma_{r}^{2} \operatorname{diag}_{9 \times 9}(1)
$$

The parameter $\sigma_{q}^{2}$ is the filter plant noise spectral density and has units of $\mathrm{m}^{2} / \mathrm{s}^{5}$, the parameter $\sigma_{r}^{2}$ is the filter measurement noise spectral density and has units of $\mathrm{m}^{2} / \mathrm{s}$

\section{- TCA Model}

The state vector for the TCA continuous time model is defined as

$$
\begin{aligned}
& X=\left[\begin{array}{lllllllll}
x & y & z & v_{x} & v_{x} & v_{x} & a_{x} & a_{y} & a_{z}
\end{array}\right] \\
& A=\left[\begin{array}{ccc}
\operatorname{diag}_{3 \times 3}(0) & R_{b e} \operatorname{diag}_{3 \times 3}(1) & \operatorname{diag}_{3 \times 3}(0) \\
\operatorname{diag}_{3 \times 3}(0) & \operatorname{diag}_{3 \times 3}(0) & R_{b e} \operatorname{diag}_{3 \times 3}(1) \\
\operatorname{diag}_{3 \times 3}(0) & \operatorname{diag}_{3 \times 3}(0) & \operatorname{diag}_{3 \times 3}(0)
\end{array}\right] \\
& C=\operatorname{diag}_{9 \times 9}(1)
\end{aligned}
$$

Discrete-time TCA model 


$$
\begin{aligned}
& X=\left[\begin{array}{lllllllll}
x & y & z & v_{x} & v_{x} & v_{x} & a_{x} & a_{y} & a_{z}
\end{array}\right] \\
& A=\left[\begin{array}{ccc}
\operatorname{diag}_{3 \times 3}(1) & R_{b e} \operatorname{diag}_{3 \times 3}(\Delta t) & R_{b e} \operatorname{diag}_{3 \times 3}\left(\frac{1}{2} \Delta t^{2}\right) \\
\operatorname{diag}_{3 \times 3}(0) & \operatorname{diag}_{3 \times 3}(1) & R_{b e} \operatorname{diag}_{3 \times 3}(\Delta t) \\
\operatorname{diag}_{3 \times 3}(0) & \operatorname{diag}_{3 \times 3}(0) & \operatorname{diag}_{3 \times 3}(1)
\end{array}\right] \\
& B=\left[\begin{array}{lll}
\frac{\Delta t^{3}}{6} & \frac{\Delta t^{2}}{2} & \Delta t
\end{array}\right]^{T}
\end{aligned}
$$

where $\Delta t$ is the difference of the measurement time

System and measurement noise convenience matrices for each state, this matrix is given as

$$
Q=\sigma_{q}^{2}\left[\begin{array}{lll}
\operatorname{diag}_{3 \times 3}\left(\frac{\Delta t^{5}}{5}\right) & \operatorname{diag}_{3 \times 3}\left(\frac{\Delta t^{4}}{4}\right) & \operatorname{diag}_{3 \times 3}\left(\frac{\Delta t^{3}}{3}\right) \\
\operatorname{diat}_{3 \times 3}\left(\frac{\Delta t^{4}}{4}\right) & \operatorname{diag}_{3 \times 3}\left(\frac{\Delta t^{3}}{3}\right) & \operatorname{diag}_{3 \times 3}\left(\frac{\Delta t^{2}}{2}\right) \\
\operatorname{diag}_{3 \times 3}\left(\frac{\Delta t^{3}}{3}\right) & \operatorname{diag}_{3 \times 3}\left(\frac{\Delta t^{2}}{2}\right) & \operatorname{diag}_{3 \times 3}(\Delta t)
\end{array}\right], R=\sigma_{r}^{2} \operatorname{diag}_{9 \times 9}(1)
$$

The parameter $\sigma_{q}^{2}$ is the filter plant noise spectral density and has units of $\mathrm{m}^{2} / \mathrm{s}^{5}$, the parameter $\sigma_{r}^{2}$ is the filter measurement noise spectral density and has units of $\mathrm{m}^{2} / \mathrm{s}$ 


\subsection{Dynamics Model of DFUAV}

The aerodynamic characteristics of the UAV determines the need for stability of the vertical plane. The UAV should have three fans for motion controlling, to load the maximum weight of the UAV at $2 \mathrm{~kg}$. In The total weight of all equipment to operate the UAV in flight should limit at $1 \mathrm{~kg}$.

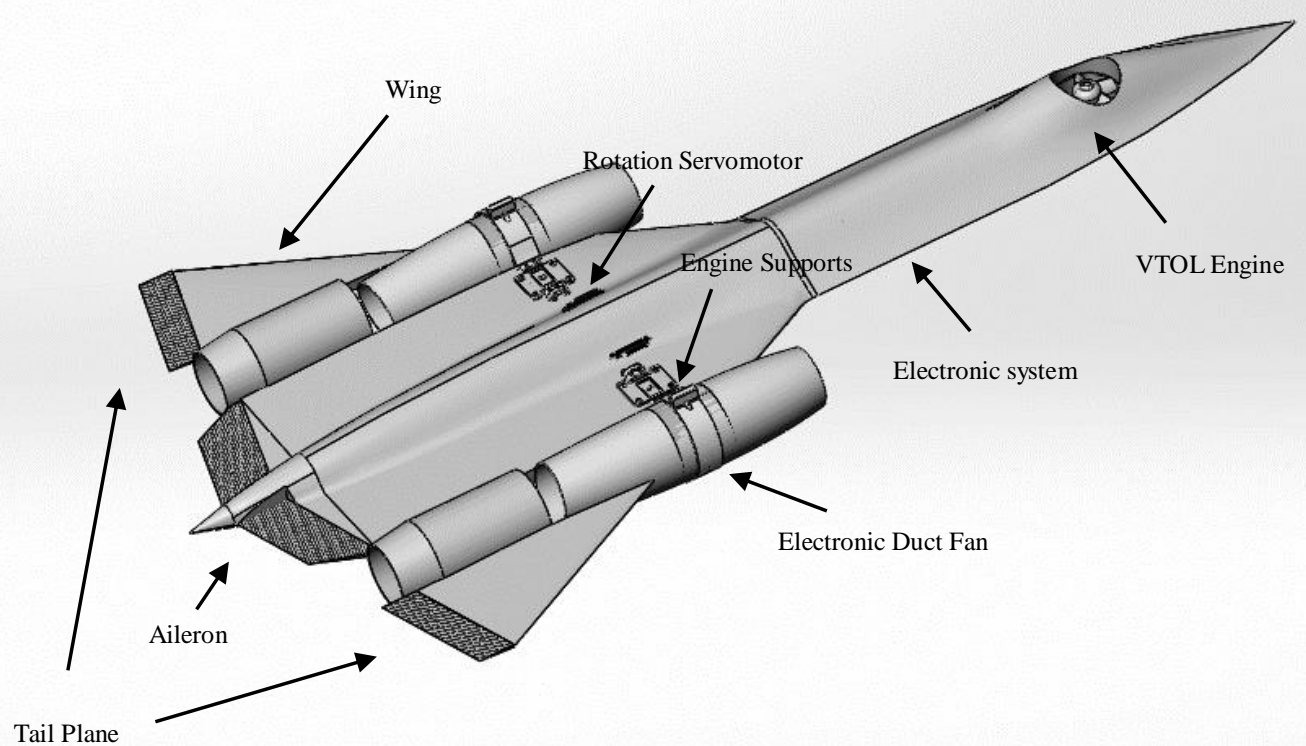

Figure 2.10 Configuration View of UAV

Table 7 UAV design characteristics

\begin{tabular}{|l|l|}
\hline Characteristic & Value \\
\hline Length of the fuselage, $\mathrm{m}$ & 1.4 \\
\hline Weight of UAV, $\mathrm{kg}$ & 1.7 \\
\hline Cruise speed, $\mathrm{m} / \mathrm{s}$ & 70 \\
\hline Minimum climb speed, $\mathrm{m} / \mathrm{s}$ & 2 \\
\hline Maximum Thrust, $\mathrm{N}$ & $25.49(2.5 \mathrm{~kg})$ \\
\hline Viscous coefficient, $\mathrm{kg} /(\mathrm{m} \cdot \mathrm{s})$ & TBD \\
\hline Ambient pressure, $\mathrm{hPa}$ & 1013.25 \\
\hline
\end{tabular}

As an aerial vehicle operated in 3 dimensional space, for each dimension, there are a pair of forces that can maintain the momentum balance: 
X-axis: Thrust \& Drag

Y-axis: Crosswind \& Side Thrust

Z-axis: Lift \& Weight

A pair of torques can maintain the angular momentum balance:

X- axis: Rolling

Y-axis: Pitching

Z-axis: Yawing

This DFUAV has three statuses transferred from take-off to horizontal flight. Each status has a unique requirement of balance.

The first states is vertical take-off. Three engines provide thrust; T1, T2 and T3, which maintains the balance of Lift and Weight, and offsets rolling and pitching. The rolling can also offset the crosswind by making the vehicle lean towards it. Two EDFs, one for engine 1 and the other for engine 2, can create vector components that thrust forward or backward. When two engines both roll the same direction, the balance of forward and backward can be maintained; when two engines roll in different directions, they can create yawing to maintain the angular momentum balance of the z-axis, as shown in Figure1.5 (a) (VTOL State of Force and Moment).

The second states is Transformation from take-off to flight. During this process, the EDFs change the angle of the engine from vertical to horizontal. This process can be reviewed as the direct thrust transformed to the lift, created by the speed of the vehicle.

The third states is horizontal flight. Thrust from 2 engines counteract the drag, and the thrust difference between two engines creates yawing for the aircraft. The rolling and pitching are controlled by the movement of flaps, and rolling can also balance the crosswind by creating horizontal lift. It is worth noting that both the lift and drag are dominated by functions which are proportional to the square of air speed. The lift and drag of wind can be calculated by wing profile, however, the lift 
and drag of the main body structure can only be simulated by the CFD, as shown in Figure1.5 (b) (Airplane State of Force and Moment).

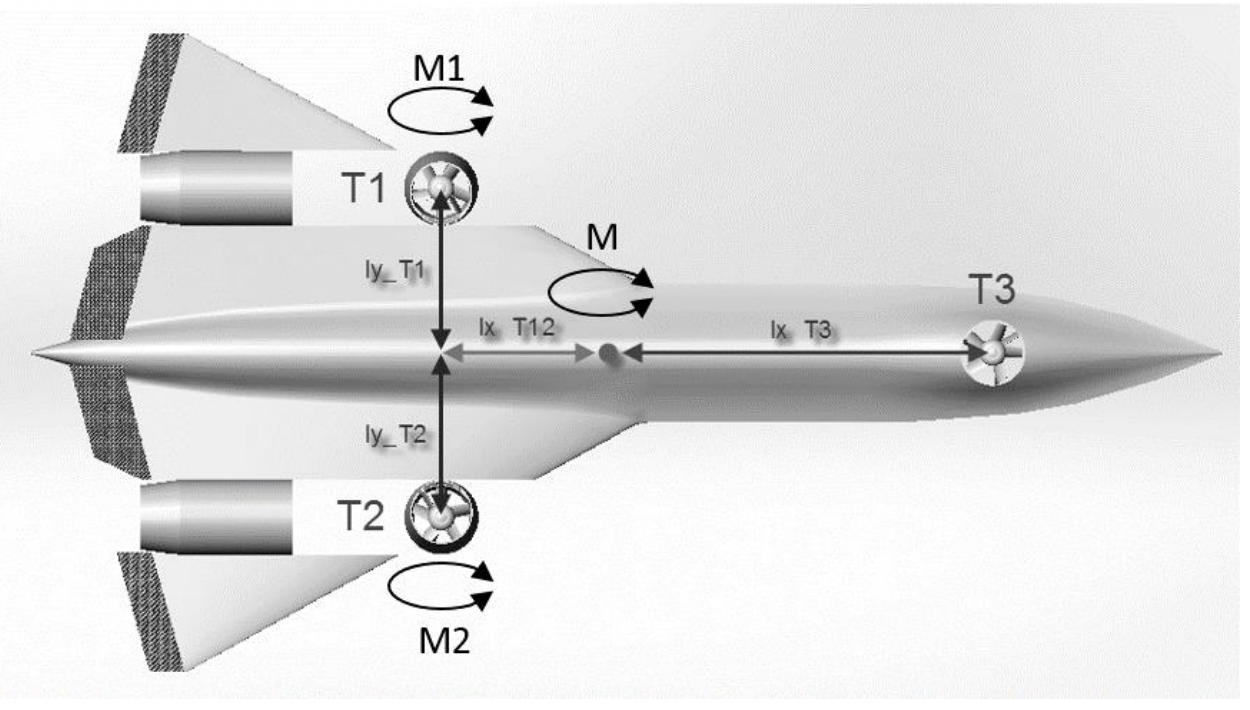

(a) VTOL State of Force and Moment

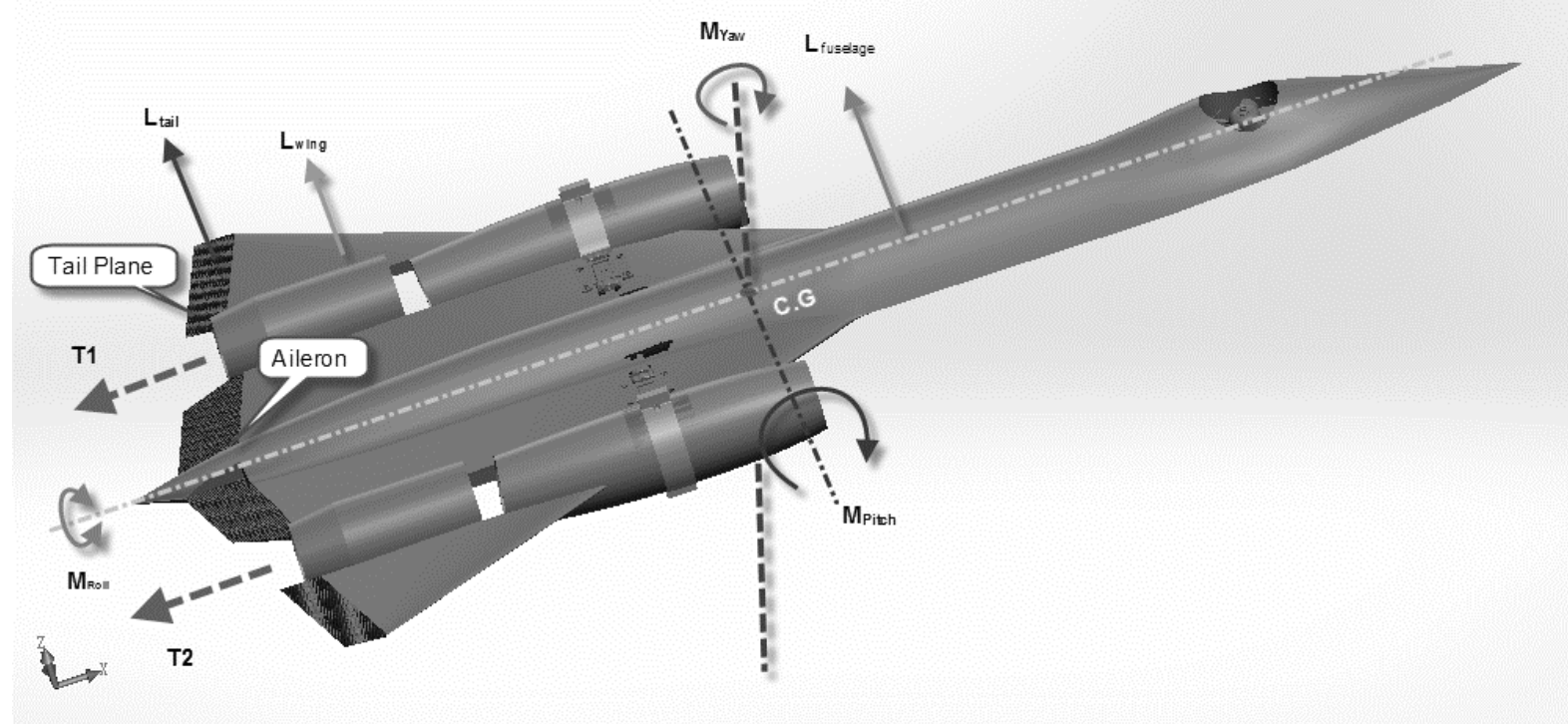

(b) Airplane State of Force and Moment

Figure 2.11 Definition of Force and Moment

Flight dynamics describes the motion of an UAV influenced by engine, airfoil or physical state. For this transmutation DFUAV, the research and development of 
stability and control abilities is important. To describe a rigid-body motion of DFUAV, the Completeness equations of motion with six degrees of freedom must be considered and analyzed.

Consider the DFUAV as a single rigid body with 6 DOFs. Assuming the earth is flat and neglecting the ground effect, the equations of motion for a rigid body subject to body force $f^{b}$ and body moment $\tau^{b}\left(f^{b}, \tau^{b} \in \mathbb{R}^{3}\right)$, applied at the center of mass and expressed in Newton-Euler formalism, are given by:

$\left[\begin{array}{l}f^{b} \\ \tau^{b}\end{array}\right]=\left[\begin{array}{cc}m & 0 \\ 0 & \mathbf{I}\end{array}\right]\left[\begin{array}{c}a^{b} \\ \dot{\omega}^{b}\end{array}\right]+\left[\begin{array}{c}\omega^{b} \times m v^{b} \\ \omega^{b} \times \mathbf{I} \omega^{b}\end{array}\right]$

Where, $\omega^{b}$ is the body angular velocity $\nu^{b}$ is the body velocity, specifies $\omega^{b}, v^{b} \in \mathbb{R}^{3}$. $\mathrm{m}$ is the total mass, $\mathbf{I} \in \mathbb{R}^{3 \times 3}$ is an inertial matrix.

a. $\omega \times m V$ is Coriolis force

b. $\omega \times I \omega$ is gyroscope torque

We ignore the Coriolis force in the DFUAV Model, because it is a small value in slowly varying motion of body. Forces of UAV are from the gravity, thrust of ducted fan, Slipstream Effect of faces and Crosswind Effect of faces. Moments of UAV also are from those effects which are forces with the forces arm. The arms are distance between the center of gravity and pressure of gravity and the distance between the center of gravity control surface.

\section{- Gravity}

The UAV frights near the ground. As a result the gravity acceleration is a negligible value than the other force with different latitude, so ignore the parameter latitude.

$$
F_{\text {gravity }}=m_{\text {uav }} g
$$




\section{- $\quad$ Thrust}

The Thrust base on the momentum has a direction as well as magnitude. The Slipstream Theory can be expressed as below (see Ref. [4])

Thrust is generated from pressure differences between region 2 and 3.

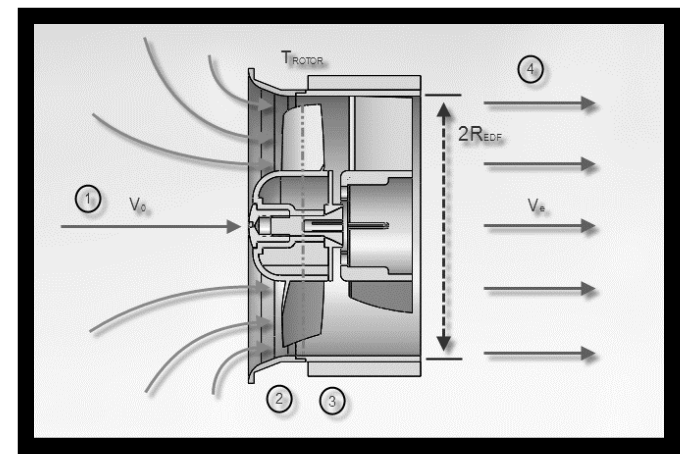

Figure 2.12 Air Flow through the Duct Fans

$T_{\text {duct }}=\dot{m}_{\text {duct }}\left(V_{\text {propeller }}+V_{0}\right)$

$\dot{m}_{\text {duct }}=\rho V_{\text {propeller }} A_{\text {duct }}$

$\mathrm{V}_{\text {propeller }}$ is induced velocity of propeller

if $V_{\text {propeller }} \gg V_{0}$

$T_{\text {duct }}=\rho A_{\text {duct }} V_{\text {propeller }}^{2}$

- Nonlinear Dynamic Equation in Earth-Axes Coordinate System VTOL state consider position controlling characteristics, the Earth -Axes Coordinate System is a direct distribution the relation VTOL motion and position location.

\section{- Newton-Euler Formalism in Earth Frame}

The Nonlinear Dynamic Equation can sprite force equation and moment equation. Above all, the forces and moments are summarized,

$$
\left\{\begin{array}{c}
F_{\text {total }}=F_{\text {thrust }}+F_{\text {gravity }} \\
M_{\text {total }}=M_{\text {rotor }}
\end{array}\right.
$$

Force equation:

$$
F_{b}=m \dot{V}_{b}+m R_{b e} g
$$


$R_{e b}=\left[\begin{array}{ccc}\cos \theta \cos \psi & \sin \theta \sin \phi \cos \psi-\cos \phi \sin \psi & \sin \theta \cos \phi \cos \psi+\sin \phi \sin \psi \\ \cos \theta \sin \psi & \sin \psi \sin \theta \sin \phi+\cos \psi \cos \phi & \sin \theta \cos \phi \sin \psi-\sin \phi \cos \psi \\ -\sin \theta & \cos \theta \sin \phi & \cos \theta \cos \phi\end{array}\right]$

Where $R_{e b}$ is rotation matrix from earth fame to body fame, $\mathrm{m}$ is mass, $\dot{V}_{b}$ is acceleration at body framing, $\mathrm{g}$ is gravity in earth framing.

Force Equation in Earth-Axes Coordinate System

$$
F_{b}=\left[\begin{array}{c}
F_{x b} \\
F_{y b} \\
F_{z b}
\end{array}\right]=\underbrace{\left[\begin{array}{c}
\sin \delta_{T_{1}} T_{1}-\sin \delta_{T_{2}} T_{2} \\
0 \\
-\left(\cos \delta_{T_{1}} T_{1}+\cos \delta_{T_{2}} T_{2}+T_{3}\right)
\end{array}\right]}_{\text {Forces control }}+R_{b e}^{\left[\begin{array}{c}
0 \\
0 \\
m g
\end{array}\right]}
$$

where $\delta_{T_{1}}, \delta_{T_{2}}$ are EDF tilt angle in body fame, $T_{1}, T_{2}, T_{3}$ is EDF thrust

Equation of Motion in earth

$$
\left[\begin{array}{c}
\dot{x}_{g} \\
\dot{y}_{g} \\
\dot{h}
\end{array}\right]=R_{e b}\left[\begin{array}{c}
F_{x b} \\
F_{y b} \\
F_{z b}
\end{array}\right]
$$

Moment equation in body frame (rotate around center of mass):

$$
\left[\begin{array}{c}
L \\
M \\
N
\end{array}\right]=J_{b o d y}\left[\begin{array}{c}
\dot{p} \\
\dot{q} \\
\dot{r}
\end{array}\right]+\underbrace{\left[\begin{array}{ccc}
0 & r & -q \\
-r & 0 & p \\
q & -p & 0
\end{array}\right] J_{b o d y}\left[\begin{array}{c}
p \\
q \\
r
\end{array}\right]}_{\text {Gyroscopic Moment }}, \quad J_{b o d y}=\left[\begin{array}{ccc}
I_{x x} & I_{x y} & I_{x z} \\
I_{y x} & I_{y y} & I_{y z} \\
I_{z x} & I_{z y} & I_{z z}
\end{array}\right]
$$

Moment of Control by engine:

$$
\left[\begin{array}{c}
L \\
M \\
N
\end{array}\right]=\underbrace{\left[\begin{array}{c}
\left(\cos \delta_{T_{1}} T_{1}-\cos \delta_{T_{2}} T_{2}\right) \cdot l_{T-G C} \\
T_{3} \cdot l_{T_{3}-G C}-\left(\cos \delta_{T_{1}} T_{1}+\cos \delta_{T_{2}} T_{2}\right) \cdot l_{T_{1} T_{2}-G C} \\
\left(\sin \delta_{T_{1}} T_{1}+\sin \delta_{T_{2}} T_{2}\right) \cdot l_{T-G C}
\end{array}\right]}_{\text {Forces control }}
$$


where $J_{\text {body }}$ is rotational inertia; $\mathrm{p}, \mathrm{q}, \mathrm{r}$ reference rotational speed $\mathrm{x}, \mathrm{y}, \mathrm{z}$ of axis. $\dot{p}$ $\dot{q} \dot{r}$ are rotational acceleration; L, M, N are moment of control by engine. $l_{T-G C}$ $l_{T_{3}-G C} l_{T_{1} T_{2}-G C} l_{T-G C}$ are distance EDF from center of gravity to EDF.

Finally Dynamic Equation:

$$
\left[\begin{array}{c}
\dot{p} \\
\dot{q} \\
\dot{r}
\end{array}\right]=J_{\text {body }}^{-1}(\underbrace{\left[\begin{array}{c}
\left(\cos \delta_{T_{1}} T_{1}-\cos \delta_{T_{2}} T_{2}\right) \cdot l_{T-G C} \\
T_{3} \cdot l_{T_{3}-G C}-\left(\cos \delta_{T_{1}} T_{1}+\cos \delta_{T_{2}} T_{2}\right) \cdot l_{T_{1} T_{2}-G C} \\
\left(\sin \delta_{T_{1}} T_{1}+\sin \delta_{T_{2}} T_{2}\right) \cdot l_{T-G C}
\end{array}\right]}_{\text {Forces control }}-\underbrace{\left[\begin{array}{ccc}
0 & r & -q \\
-r & 0 & p \\
q & -p & 0
\end{array}\right] J_{b o d y}\left[\begin{array}{c}
p \\
q \\
r
\end{array}\right]}_{\text {Gyroscopic Moment }})
$$

Equation of Motion in Euler angle:

$$
\begin{aligned}
\dot{\phi} & =p+\tan \theta(q \sin \phi+r \cos \phi) \\
\dot{\theta} & =q \cos \phi-r \sin \phi \\
\dot{\psi} & =\frac{1}{\cos \theta} q(\sin \phi+r \cos \phi)
\end{aligned}
$$

where $\dot{\phi} \dot{\theta} \dot{\psi}$ are reference rotational speed at END(North, East, Down) earth framing.

\section{GD Filter for Attitude Estimation}

Gradient descent algorithm [5] is a liner regression algorithm is used Artificial Intelligence domain to find a local minimum. The paper use the algorithm to fusion IMU's tri-axis gyroscopes, accelerometers and magnetometers.

These are several processing. Firstly, we need to get a direction of the gradient $\frac{\nabla f}{\|\nabla f\|}$. It essentially uses the quaternion of previous moment ${ }_{E}^{B} \hat{q}_{t},{ }_{E}^{B} \hat{q}_{t} \in \Re^{4}$ to 
calculate the virtual acceleration and magnet values. Secondly, get the acceleration and magnetometers errors $f_{g, m}\left({ }_{E}^{B} \hat{q},{ }^{B} \hat{a},{ }^{B} \hat{m}\right)$ through using actual acceleration and magnetometers to minus the virtual values. Thirdly, transfer these parameters to error of quaternion gradient by the Jacobian matrix $J_{g, m}\left({ }_{E}^{B} \hat{q}_{t-1}\right)$. Renew the quaternion of the next moment ${ }_{E}^{B} \dot{\hat{q}}_{e, t-1}$ by the equation ${ }_{E}^{B} \hat{q}_{t}={ }_{E}^{B} \hat{q}_{t-1}+\Delta t\left({ }_{E}^{B} \dot{q}_{\omega, t}-{ }_{E}^{B} \dot{\hat{q}}_{e, t-1}\right)$. It uses the gradient information of quaternion errors to iterate so that it makes the error become 0 . The down velocity is depends on the first order partial derivative value. $\beta$ is the learning rate. Finally, if the GD algorisms what the prerequisites are not stationary state, the algorism will contain extra acceleration and magnetic field changes. so a Motion detection function is used to turn off/on correcting signal when acceleration and magnetometers value change. Their pdf value were used identify motion change. 


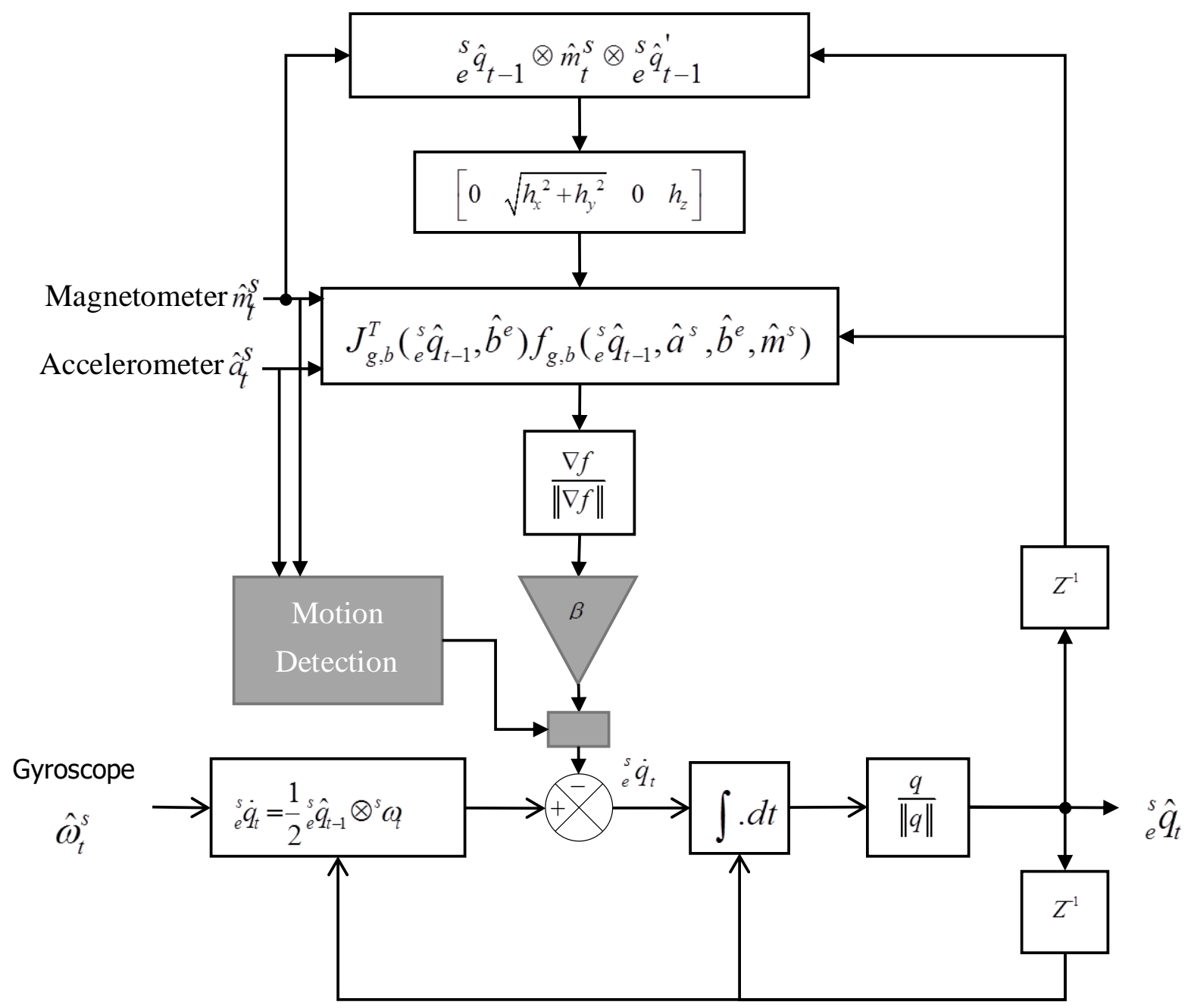

Figure 3.1 Gradient Descent Algorithm Block Diagram

- Orientation from angular rate

$$
\begin{aligned}
& { }_{E}^{B} \dot{q}_{\omega, t}=\frac{1}{2}{ }_{E}^{B} \hat{q}_{t-1} \otimes{ }^{B} \omega_{t} \quad \omega_{t} \in \mathfrak{R}^{3} \\
& \omega_{t} \text { : Values of } \mathrm{x}, \mathrm{y}, \mathrm{z} \text { axis gyroscopes. }
\end{aligned}
$$

Update next time quaternion ${ }_{E}^{B} \hat{q}_{t}$ use integration law.

$$
{ }_{E}^{B} \hat{q}_{t}={ }_{E}^{B} \hat{q}_{t-1}+{ }_{E}^{B} \dot{q}_{d e s, t} \Delta t
$$

\section{- Gradient from error of accelerometer and magnetometers}




$$
\begin{aligned}
& f_{g}\left({ }_{E}^{B} \hat{q},{ }^{B} \hat{a}\right)=\left[\begin{array}{c}
2\left(q_{2} q_{4}-q_{1} q_{3}\right) \\
2\left(q_{1} q_{2}+q_{3} q_{4}\right) \\
2\left(\frac{1}{2}-q_{2}^{2}-q_{3}^{2}\right)
\end{array}\right]-\left[\begin{array}{l}
a_{x} \\
a_{y} \\
a_{z}
\end{array}\right] \\
& J_{g}\left({ }_{E}^{B} \hat{q}\right)=\left[\begin{array}{cccc}
-2 q_{3} & 2 q_{4} & -2 q_{1} & 2 q_{2} \\
2 q_{2} & 2 q_{1} & 2 q_{4} & 2 q_{3} \\
0 & -4 q_{2} & -4 q 3 & 0
\end{array}\right] \\
& \nabla f=f_{g}\left({ }_{E}^{B} \hat{q},{ }^{B} \hat{a}\right) J_{g}\left({ }_{E}^{B} \hat{q}\right) \\
& { }_{E}^{B} \dot{\hat{q}}_{e, t-1}=\frac{\nabla f}{\|\nabla f\|}
\end{aligned}
$$

Gradient update law:

$$
{ }_{E}^{B} \dot{q}_{d e s, t}={ }_{E}^{B} \dot{q}_{\omega, t}-\beta{ }_{E}^{B} \dot{\hat{q}}_{e, t-1}
$$

Rotation matrix transfer earth frame to body frame:

$$
R_{b e}=\left[\begin{array}{ccc}
q_{0}^{2}+q_{1}^{2}-q_{2}^{2}-q_{3}^{2} & 2\left(q_{1} q_{2}+q_{0} q_{3}\right) & 2\left(q_{1} q_{3}-q_{0} q_{2}\right) \\
2\left(q_{1} q_{2}-q_{0} q_{3}\right) & q_{0}^{2}-q_{1}^{2}+q_{2}^{2}-q_{3}^{2} & 2\left(q_{2} q_{3}+q_{0} q_{1}\right) \\
2\left(q_{0} q_{2}+q_{1} q_{3}\right) & 2\left(q_{2} q_{3}-q_{0} q_{1}\right) & q_{0}^{2}-q_{1}^{2}-q_{2}^{2}+q_{3}^{2}
\end{array}\right]
$$

Beacuse: $q_{0}^{2}+q_{1}^{2}+q_{2}^{2}+q_{3}^{2}=1$

$$
R_{b e}=\left[\begin{array}{ccc}
2 q_{0}^{2}+2 q_{1}^{2}-1 & 2\left(q_{1} q_{2}+q_{0} q_{3}\right) & 2\left(q_{1} q_{3}-q_{0} q_{2}\right) \\
2\left(q_{1} q_{2}-q_{0} q_{3}\right) & 2 q_{0}^{2}+2 q_{2}^{2}-1 & 2\left(q_{2} q_{3}+q_{0} q_{1}\right) \\
2\left(q_{0} q_{2}+q_{1} q_{3}\right) & 2\left(q_{2} q_{3}-q_{0} q_{1}\right) & 2 q_{0}^{2}+2 q_{3}^{2}-1
\end{array}\right]
$$

Euler angles from quaternion:

$$
\begin{aligned}
& \theta_{d}=\arcsin \left(-2\left(q_{1} q_{3}-q_{0} q_{2}\right)\right) \\
& \phi_{d}=\arctan \left(\frac{2\left(q_{2} q_{3}+q_{0} q_{1}\right)}{2 q_{0}^{2}+2 q_{3}^{2}-1}\right) \\
& \psi_{d}=\arctan \left(\frac{2\left(q_{1} q_{2}+q_{0} q_{3}\right)}{2 q_{0}^{2}+2 q_{1}^{2}-1}\right)
\end{aligned}
$$

Motion detection function 
The likelihood functions for acceleration model is as follows:

$$
\begin{aligned}
P D F & =\mathcal{N}\left(A_{\text {nom }} ; 0 ; \sigma^{2}\right) \\
& =\frac{1}{\sqrt{2 \pi^{2}} \sigma} e^{-\frac{A_{\text {nom }}^{2}}{2 \sigma^{2}}} \\
A_{\text {nom }} & =\sqrt{a c c_{x}+a c c_{y}+a c c_{z}}
\end{aligned}
$$

Where $A_{\text {nom }}$ is acceleration modulus, $A_{\text {nom }}$ is the covariance matrix, $a c c_{x}, a c c_{y}, a c c_{z}$ is acceleration sensor data.

\subsection{Simulation and Result}

A flight signal generator is needed which combine inertial navigation system, GPS system and magnetic system, each block can setup noise parameters as Rate random walk, correlation time and measure noise. The detail show in Figure

\begin{tabular}{|c|c|}
\hline Function Block Parameters: Inertial Navigation System & $x$ \\
\hline Subsystem (mask) & 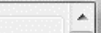 \\
\hline Gyroscope Accelerometer & \\
\hline Acc Rate Random Walk (K) & \\
\hline$[0.0001 ; 0.0001 ; 0.0001 ;]$ & \\
\hline Acc Measurement Noise (AR) & \\
\hline$[0.005 ; 0.005 ; 0.005 ;]$ & $=$ \\
\hline Accelerometer upper and lower limits: & \\
\hline$\left[\begin{array}{llllll}-18 & -18 & -18 & 18 & 18 & 18\end{array}\right]$ & \\
\hline Gravitational Acceleration & \\
\hline 9.78 & \\
\hline Noise correlation time (sec) & \\
\hline$[14400: 14400: 14400:]$ & 4 \\
\hline Cancel & Apply \\
\hline
\end{tabular}
8 (Flight Signal Generator Noise Parameters)

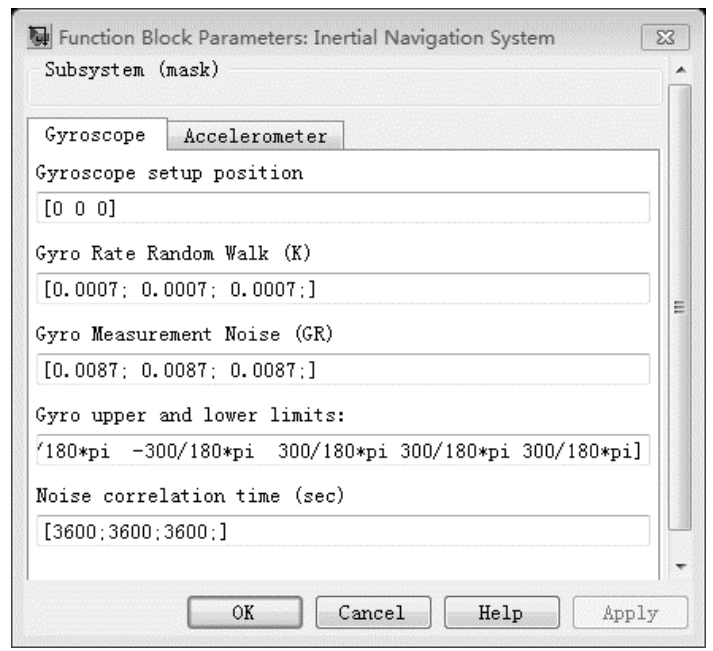



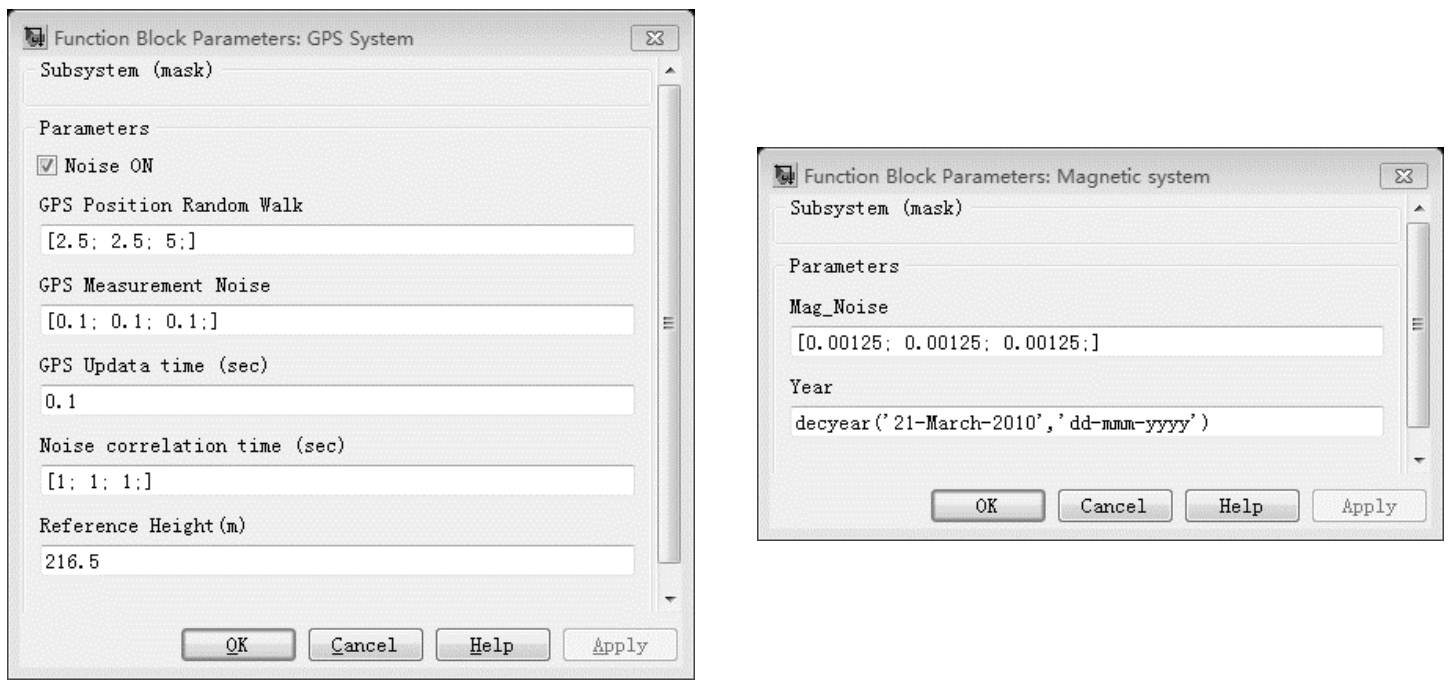

Figure 3.2 Flight Signal Generator Noise Parameters

Gradient Descent Algorithm (GDA) confirmation is need do the test which input a signal with noises to observe module response in MATLAB simulation for 200 second. A group stationary state data of magnetic, gyroscope, acceleration are used an excitation source. Figure 9 (Sensor Data Input) show the sensor data input. Different Learning Rates are tested By RMS at Figure 9. Figure 10 (GDA Attitude Estimate output) show the attitude estimate output. Yellow line shown pith angle data. Pink line shown roll angle data. Cyanic shown yaw angle data. The error range of angle data in $\pm 0.04^{\circ}$. So the Figure show that if learning rate $\beta$ is set at 0.004 , the GD Algorithm will get the minimum RMSE. 

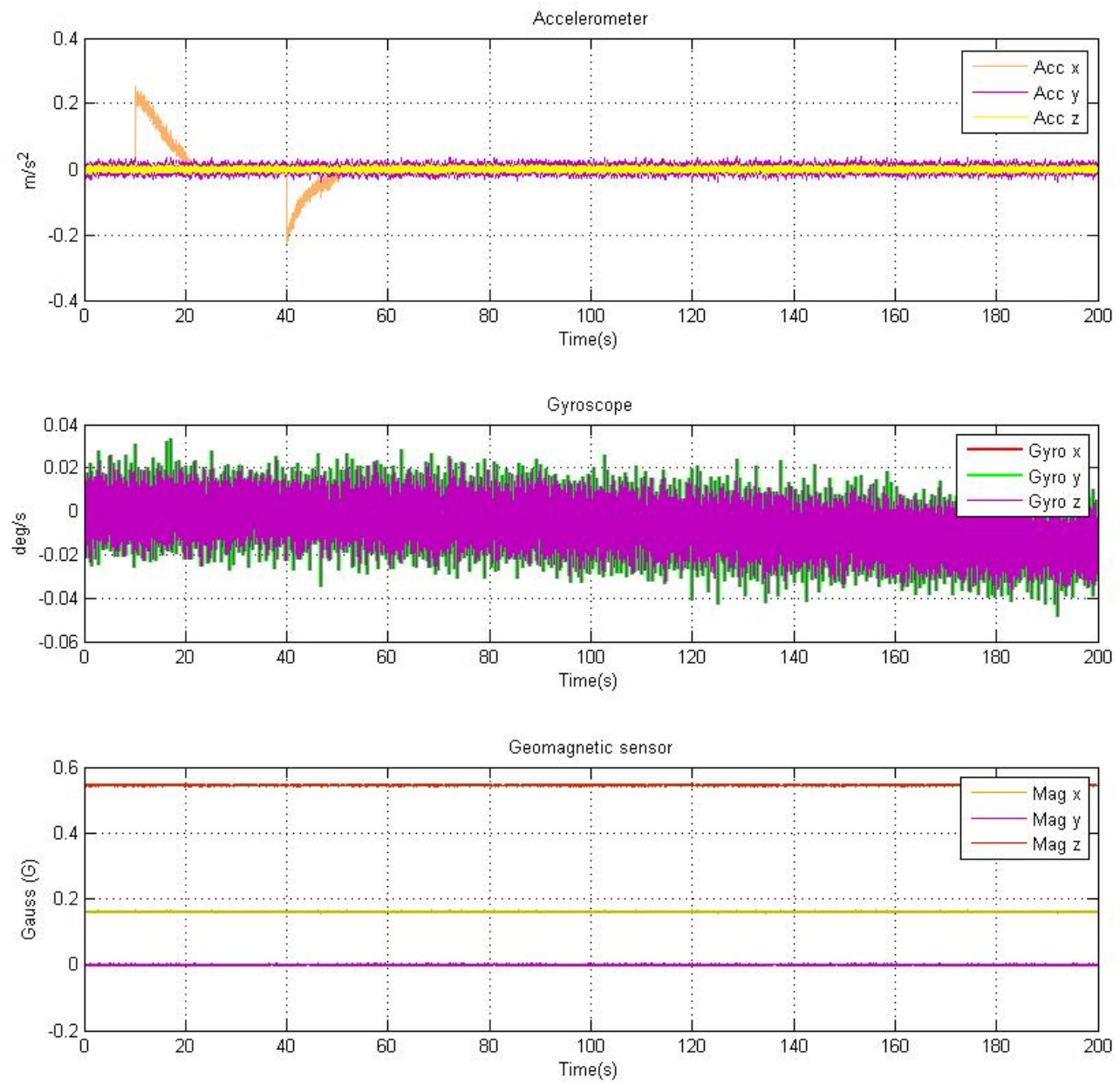

Figure 3.3 Sensor Data Input 


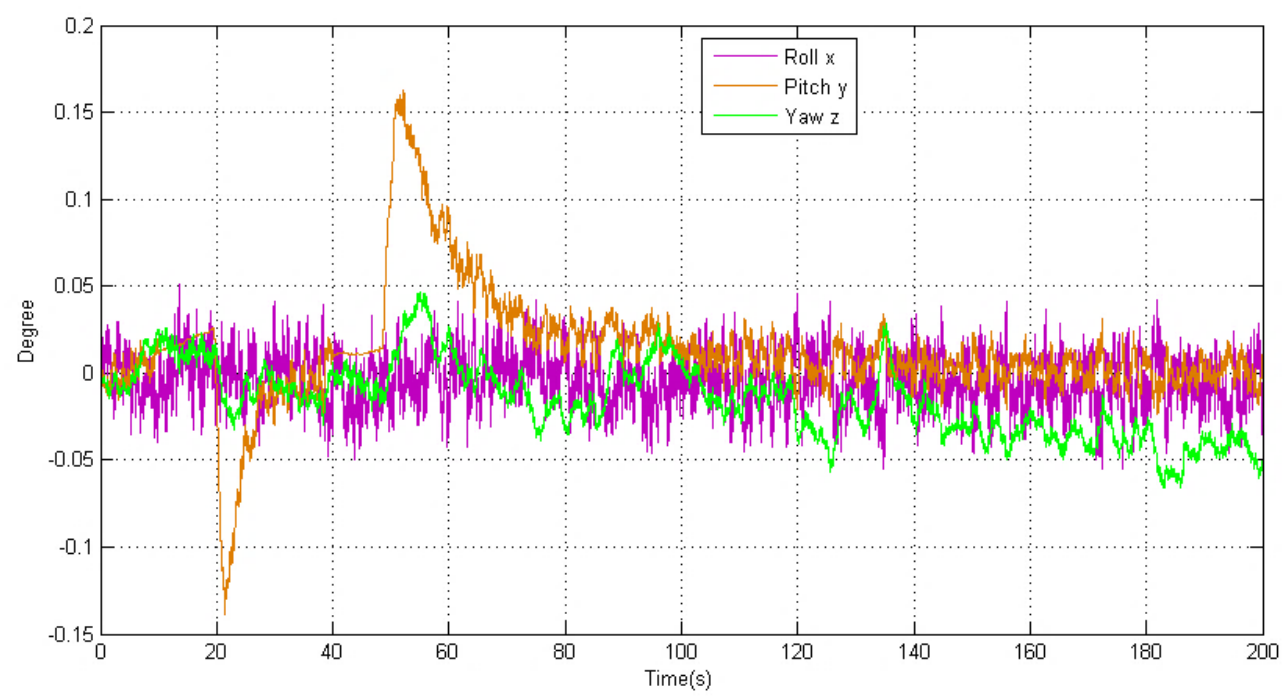

Figure 3.4 GDA Attitude Estimate Output

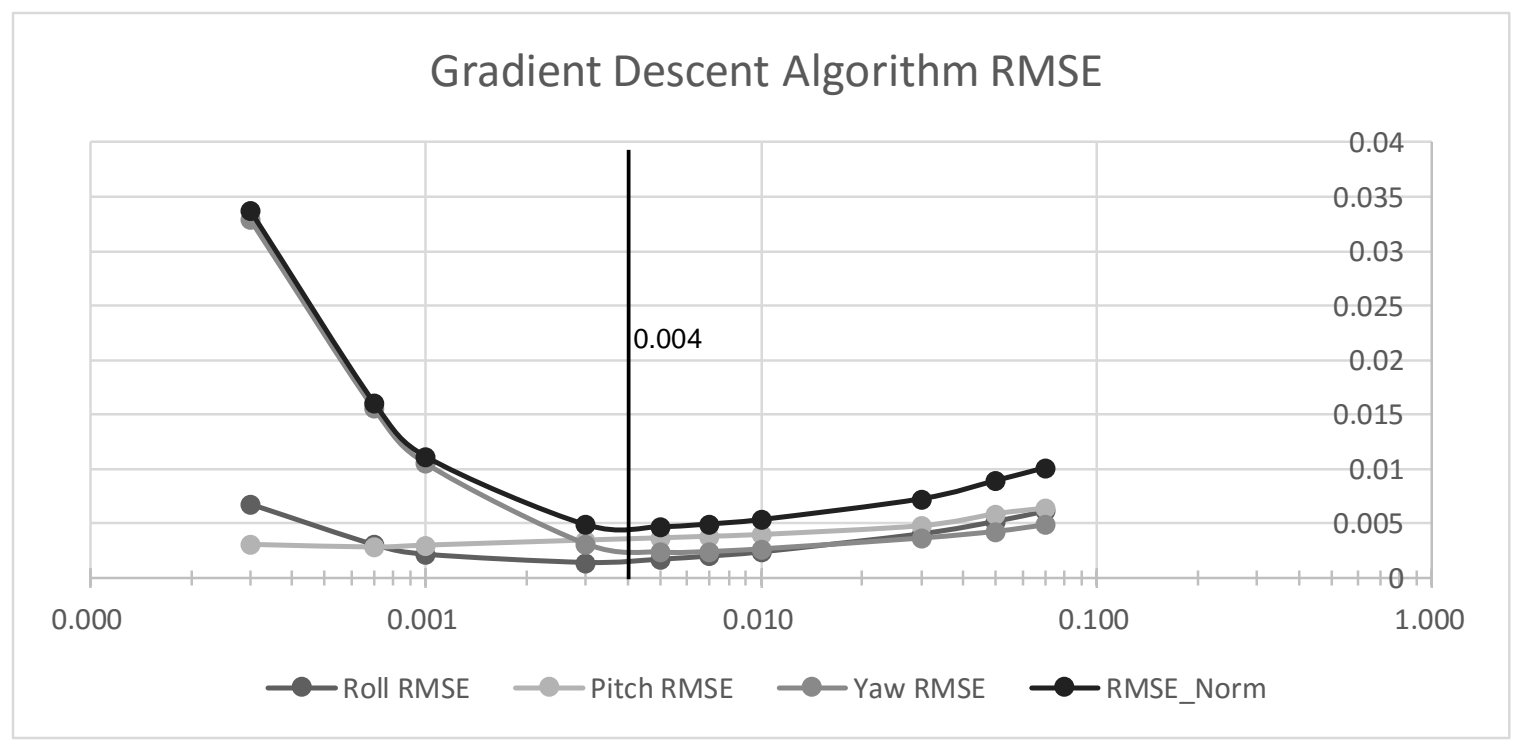

Figure 3.5 RMSE Testing for Different Learning Rates $\beta$ 


\section{IMM Filter for Position Estimation}

\subsection{IMM Filter Description}

When set up estimation models, considering mathematical modeling assumptions the only uncertainty is by white noise process and measurement with known additive statistical properties. In other words, the system model, the state transition matrix, input gain, the measurement noise covariance matrix and are assumed to be known. But the reality is that because of the internal noise superimposed on the sensor, such as generating a random walk noise by gyroscope; GPS measurements in reflection and refraction of radio waves to produce multipath effect; the impact of the geomagnetic sensor that is fixed external magnetic interference tendency bias, and ambient wind generation aircraft involuntary displacement. It will cause the measurement system will introduce a variety of time-varying color noise. Therefore, system modeling will be a combination of system parameter uncertainties with unknown inputs where the system parameters (are assumed to) take values in a discrete set.

$X(k+1)=F(k) x(k)+G(k) u(k)+v(k)$

but the input $u(k)$, which enters the $v(k)$, is unknown, $F(k)$ is time varying, it is desirable to establish an Adaptive estimation algorithms in real-time assessment of noise $w$ change and the system state changes.

Considering above condition UAV belong to a random process Dynamic Multiple Model. As shown in the table for such a system can use several discrete levels with merging or switching between them.

Thus we may consider the system, the current system is composed of a discrete group (contains $n$ models), which is remarked $M=\{M 1, \ldots, M n\}$. We assume that each model is $\mathrm{Mj}$, and we have a model corresponding prior probability $\mathrm{u}=\mathrm{P}$ $\{M\}$.switched from model $i$ to model $j$, the probability in the next moment is assumed to be known, and remarked Pij. This can be seen as a first order Markov chain model mode conversion process, this type of system is commonly referred to as Markovian switching systems. The best way to take advantage of 
this state of the plurality of filter to estimate a model system, we must require every optimal filter records of all state sequence, In other words, the optimal filter processing kth measurements, for $n$ model requires recording $n^{k}$ message. Therefore, in order to avoid such a doubling of the historical record storage, need some approximation in the practical application of multiple model systems, actually this is a suboptimal techniques.

A simple suboptimal techniques approach is record the maximum probability in $\mathrm{n}$ histories, other information discarded and re-normalized probability, making them add up to 1. Suboptimal techniques filtering problem is generalized PseudoBayesian (GPB) algorithm, Generalized Pseudo-Bayesian (GPB) approach combines many historical model that "old" models. First Order 'GPB, expressed as GPB1, considering only the past time possible model. Second Order, GPB2, considering all possible model past two moments. These two algorithms require $r$ and $2 r$ filters operate respectively in parallel. (See Ref [6], [7])

The Interacting Multiple Model (IMM) estimator algorithm is a dynamic multiple model estimator. The algorithm assumes that the system behaves according to one of a finite number of models - it is in one of several modes (operating regimes). The models can differ in noise levels or their structure - different state dimensions and unknown inputs can be accommodated as well. Such system is called a hybrid system - it has both discrete (structure/parameters) and continuous uncertainties (additive noises). This algorithm is conceptually similar to GPB2, but requires only $r$ filters to operate in parallel (See Ref [8]) as Figure 4.1 shown frameworks.

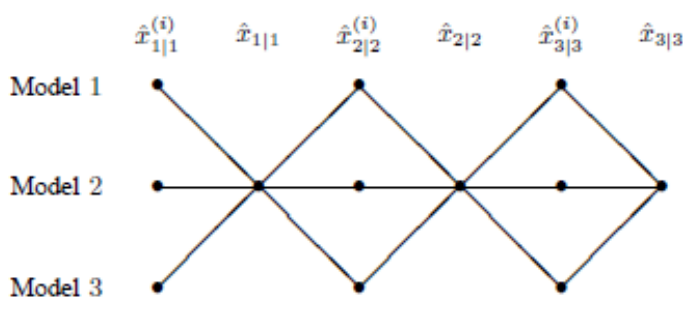

(a) GPB1

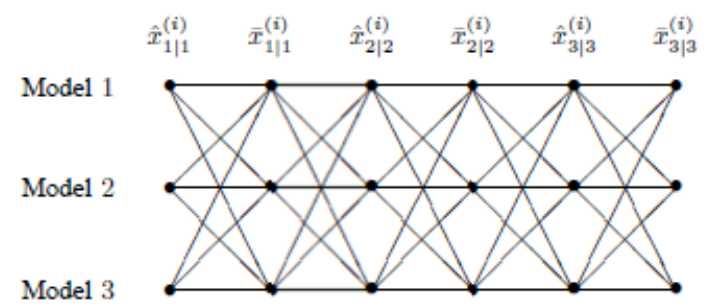

(b) GPB2 


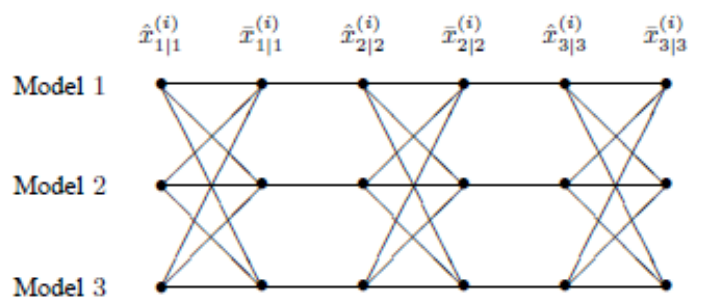

(c) IMM

Figure 4.1 GPB1, GBP2 and IMM frameworks

Comparison of complexities of the MM algorithms.

Table 8 Compare different computation complexity with different algorithm

\begin{tabular}{|l|l|l|l|l|}
\hline & Static & GPB1 & GPB2 & IMM \\
\hline Number of filters & $r$ & $r$ & $r^{2}$ & $r$ \\
\hline $\begin{array}{l}\text { Number of combinations of } r \text { estimates and } \\
\text { covariances }\end{array}$ & 1 & 1 & $r+1$ & $r+1$ \\
\hline Number of probability calculations & $r$ & $r$ & $r^{2}+r$ & $r^{2}+r$ \\
\hline
\end{tabular}

In a research paper [9] [10], author publish the "steady-state" errors during nonmaneuvering obtained and the average errors during maneuver obtained by time averaging by time averaging in Table 9 and Table 10.

Table 9 Average RMSEs during Non-Maneuvering

\begin{tabular}{|l|l|l|l|}
\hline & GPB1 & GPB2 & IMM \\
\hline Position $(\mathrm{m})$ & 79.9 & 63.3 & 65.8 \\
\hline Velocity $(\mathrm{m} / \mathrm{s})$ & 18.5 & 10.4 & 11.1 \\
\hline
\end{tabular}

Table 10 Average Position \& Velocity RMSEs during Maneuver

\begin{tabular}{|l|l|l|l|}
\hline & GPB1 & GPB2 & IMM \\
\hline Position $(\mathrm{m})$ & 152.7 & 116.7 & 118.1 \\
\hline Velocity $(\mathrm{m} / \mathrm{s})$ & 111.6 & 79.1 & 79.8 \\
\hline
\end{tabular}


In terms of position error the algorithms rank as follows: GPB2-best, IMM-middle, and GPB1-worst.

An execution time testing are followed. The execution times for the algorithms relative to the IMM are given in Table 11.

Table 11 Relative Execution Time

\begin{tabular}{|l|l|l|l|}
\hline & GPB1 & GPB2 & IMM \\
\hline $\begin{array}{l}\text { Execution Time } \\
(\mathrm{s})\end{array}$ & 1.09 & 7.91 & 2.30 \\
\hline
\end{tabular}

All in this article, we use IMM estimator algorithm, to achieve complex DFUAV Navigation System.

\subsection{Design of IMM Filter}

\subsubsection{IMM Filter Process Framework}

Processing of the IMM algorithm is shown in figure one. A mixing probabilities is using moment matching to GPB2 Gaussian mixture models one method of Approximation by a single Gaussian. Interaction of different models mixture with weightings create the new $x$ and $P$. Then use EKF state estimation of Interaction $x$ get a new, $x$ and $P$. Used likelihood functions corresponding to the different filters, Then using Bayesian total probability formula update each new measurement, and the resulting weighting factors are used in calculating the state. Finally, for Gaussian mixture models we can use mixture probability density function combined the status of each model according to the model probabilities. We can get mixture mean $\hat{X}_{1_{-} \text {mix }}^{\mathrm{k}}$ and mixture covariance $\hat{P}_{1_{-} \text {mix }}^{k}$. One cycle of a practical IMM algorithm consists of the following steps [11] [12] [13].

The IMM estimator have five process in one cycle. [12]

- Calculation of the mixing probabilities.

- Interaction/Mixing.

- Mode-matched filtering. 
- Mode probability update.

- Estimate and covariance combination.

The following section describes the specific core algorithms on IMM total of 4 models: Model Switch Algorithm, Interacting multiple model estimation, Modematched filtering.

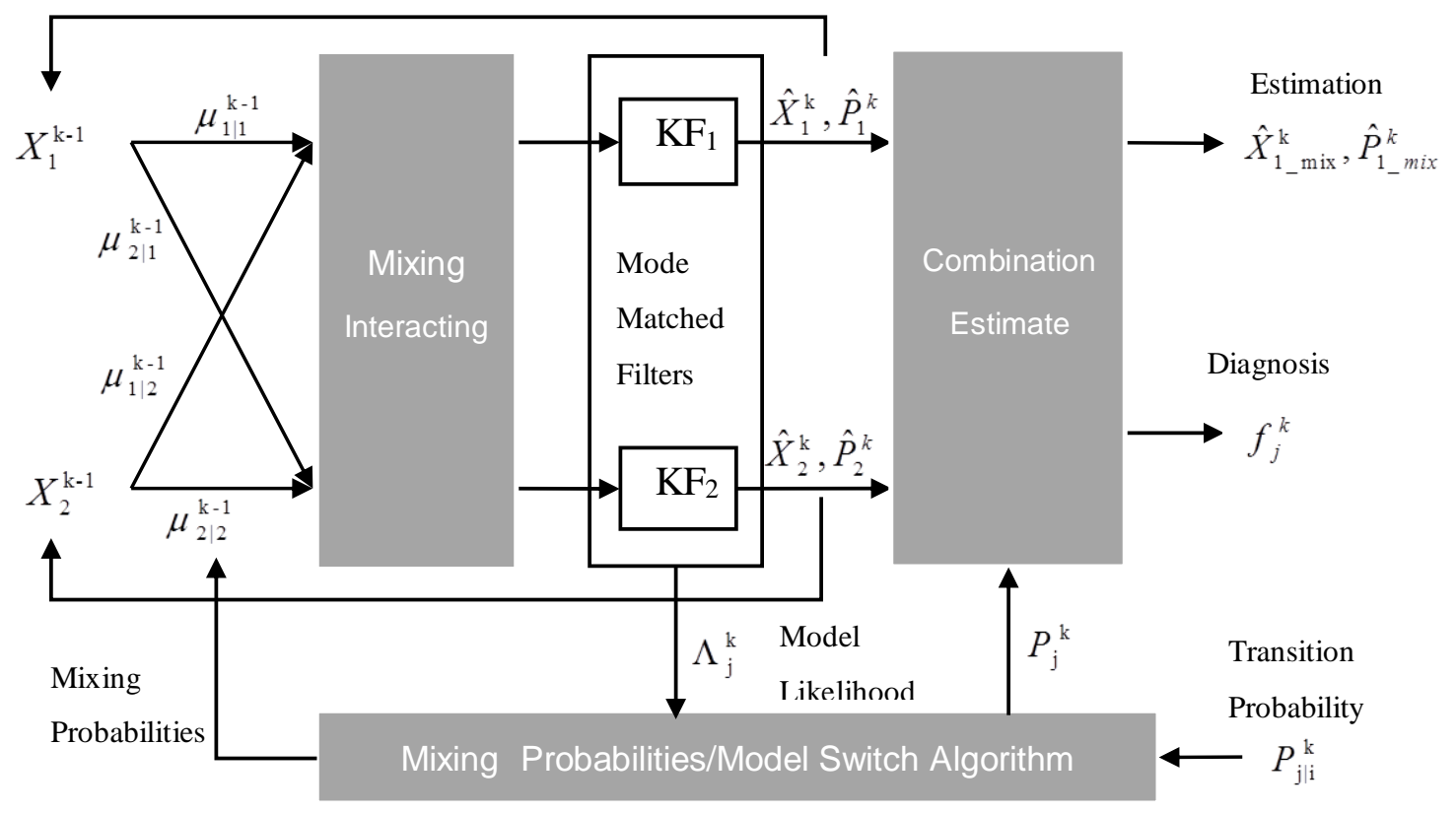

Figure 4.2 Block diagram for the IMM Algorithm

\subsubsection{IMM Models Bank}

In this case the mode the system is in can undergo switching in time. The state of UAV System models are denoted as equation for different sensor subsets:

$$
\begin{aligned}
& x(k)=F[M(k)] x(k-1)+G[M(k)] u(k-1)+w[k-1, M(k)] \\
& z(k)=H[M(k)] x(k)+v[k, M(k)]
\end{aligned}
$$

where $x \in R^{n_{x}}$ is the system state vector, $z \in R^{n_{z}}$ is the measurement vector, $\mathrm{u} \in R^{n_{\mathrm{u}}}$ and $v \in R^{n_{z}}$ are mutually independent, white zero mean Gaussian noises with normal 
distribution $N\left(0, \sigma^{2}\right)$. The parameter $M(k)$ presents the current system model. $F$ is the system dynamic matrix, and $H$ is the measuring matrix. Because the current system model is unknown, the system is described by possible $\mathrm{n}$ modes.

$$
M(k) \in\left\{M_{j}\right\}_{j=1}^{n}
$$

So the discrete model at one time can denote as:

$x(k)=F\left[M_{j}\right] x(k-1)+G\left[M_{j}\right] u(k-1)+w\left[k-1, M_{j}\right]$

$z(k)=H\left[M_{j}\right] x(k)+v\left[k, M_{j}\right]$

The system noise is $w\left[k-1, M_{j}\right] \sim \mathcal{N}_{w}\left(\mu_{j}, Q_{j}\right)$,

The measurement noise is $v\left[k, M_{j}\right] \sim \mathcal{N}_{v}\left(\mu_{j}, Q_{j}\right)$

The th mode history - or sequence of models - through time $\mathrm{k}$ is denoted as

$$
M^{k, l}=\left\{M_{i_{1, l}}, \cdots M_{i_{k, l}}\right\} \quad l=1, \ldots, n^{k}
$$

where $i_{k, l}$ is the model index at time $k$ from history / and

$$
1<i_{k, l} \leq n \quad k=1, \ldots, k
$$

It is assumed that the system mode (model) switching is a Homogeneity Markov Chain. The $p_{j \mid i}$ is transition probabilities

$$
\begin{aligned}
& p_{j \mid i} \triangleq P\left\{M(k)=M_{j} \mid M(k-1)=M_{i}\right\} \\
& \text { where } \sum_{j=1}^{n} p_{j \mid i}=1, \quad \mathrm{i}=1,2 \ldots \ldots, \mathrm{n}
\end{aligned}
$$

\subsubsection{Calculate the Mixing Probabilities}

The mixing probability $p_{i \mid j}^{k-1}$ for mode $m_{i}$ was effect at time k-1 given that $m_{j}$ is in effect at time $\mathrm{k}$ conditioned on $Z^{k-1}$ is 


$$
\begin{aligned}
\mu_{i \mid j}^{k-1} & =P\left\{m_{i}(k-1) \mid m_{j}(k), Z^{k-1}\right\} \\
& =\frac{p_{j \mid i} \mu_{j}^{k-1}}{\sum_{i=1}^{n} p_{j \mid i} \mu_{j}^{k-1}}
\end{aligned}
$$

where $\bar{p}_{j}=\sum_{j=1}^{n} p_{j \mid i}^{k} p_{j}^{k-1}$ is the predicted mode probabilities and $\mathrm{n}$ different modes.

\subsubsection{Calculate the Mixed Initial Condition}

A mixing probabilities is using moment matching to GPB2 Gaussian mixture models approach an Approximation by a single Gaussian (See Ref [14]).

Interaction to the different models mixture with weightings $\mu_{i \mid j}^{k-1}$ create new $\hat{x}_{j}^{k-1}$

end $\hat{P}_{j}^{k-1}$.

Minute probability density function:

$P(x)=\sum_{j=1}^{n} \mu_{j} \mathcal{N}\left(x ; \hat{x}_{j}, P_{j}\right)$

Starting with previous state estimates $\hat{x}_{j}^{k-1}$, and $\hat{P}_{j}^{k-1}$ previous covariance matrices obtained as output from the $\mathrm{n}$ different Kalman filters (acting as the $\mathrm{n}$ different modes).

Mixed initial condition for the filter $m_{j}$ at time $\mathrm{k}$ is:

$$
\begin{aligned}
& \hat{x}_{j}^{k-1}=\sum_{i=1}^{n} \hat{x}_{i}^{k-1} \mu_{i \mid j}^{k-1} \quad j=1, \ldots, n \\
& \hat{P}_{j}^{k-1}=\sum_{i=1}^{n} \mu_{i \mid j}^{k-1}\left\{P_{i}^{k-1}+\left[\hat{x}_{i}^{k-1}-\hat{x}_{j}^{k-1}\right]\left[\hat{x}_{i}^{k-1}-\hat{x}_{j}^{k-1}\right]^{T}\right\} \quad j=1, \ldots, n
\end{aligned}
$$

\subsubsection{Mode-Matched Filtering and Likelihood Function}

Kalman filter is used to estimate the state for each model as call mode-matched filtering, it is an important part of IMM algorithm, which can be estimated ${ }^{\hat{x}_{i}^{k-1}}, \hat{P}_{i}^{k-1}$ and $\Lambda_{\mathrm{j}}^{\mathrm{k}}$. A basic 
assumption of the Kalman filter is to measure noise and process noise is Gaussian distribution and uncorrelated. Eight Kalman filter equations can be divided into two parts, forecasts and updates. Prediction section includes former two equations and updated part by the 3 to 8 equation. [14]

Step Kalman filter is as follows:

Predict:

$$
\begin{array}{ll}
x_{k \mid k-1}=F_{k} x_{k-1 \mid k-1}+B_{k} u_{k} & \text { Predicted (a priori) state estimate } \\
P_{k \mid k-1}=F_{k} P_{k-1 \mid k-1} F_{k}^{\mathrm{T}}+Q_{k} & \text { Predicted (a priori) estimate covariance }
\end{array}
$$

Update:

$$
\begin{array}{ll}
y_{k}=z_{k}-H_{k} x_{k \mid k-1} & \text { Innovation or measurement residual } \\
S_{k}=H_{k} P_{k \mid k-1} H_{k}^{T}+R_{k} & \text { Innovation (or residual) covariance } \\
K_{k}=P_{k \mid k-1} H_{k}^{T} S_{k}^{-1} & \text { Optimal Kalman gain } \\
x_{k \mid k}=x_{k \mid k-1}+K_{k} y_{k} & \text { Updated (a posteriori) state estimate } \\
P_{k \mid k}=\left(I-K_{k} H_{k}\right) P_{k \mid k-1} & \text { Updated (a posteriori) estimate covariance }
\end{array}
$$

$y_{k}, S_{k}$ are the innovation process and its covariance matrix; $K_{k}$ the Kalman filter gain.

$y_{k}, S_{k}$ are the innovation process and its covariance matrix; $K_{k}$ the Kalman filter gain.

The likelihood functions for filter $\mathrm{j}$ is as follows:

$$
\begin{aligned}
\Lambda_{\mathrm{j}}^{\mathrm{k}} & =\mathcal{N}\left(y_{k}^{j} ; 0 ; S_{k}^{j}\right) \\
& =\frac{1}{\sqrt{2 \pi S_{k}^{j}}} e^{-\frac{\left(y_{k}^{j}\right)^{T} \cdot y_{k}^{j}}{2 S_{k}^{j}}}
\end{aligned}
$$

Where $y_{k}^{j}=z_{k}-z_{k \mid k-1}$ is the innovation for filter $j$ and $S_{k}^{j}$ is the covariance matrix associated with $y_{k}$ 


\subsubsection{Update Model Probability}

$\mu_{j}^{k}=\frac{1}{c} \Lambda_{j}^{k} \cdot \bar{p}_{j}^{k-1}$ where $c=\sum_{j=1}^{n} \Lambda_{j}^{k} \cdot \bar{p}_{j}^{k-1}$

\subsubsection{Combine Model- Conditioned Estimates and Covariance}

A Gaussian mixture model is a weighted sum of $\mathrm{n}$ component Gaussian densities as given by the equation,

$P(x)=\sum_{j=1}^{n} w_{j} \mathcal{N}\left(x ; \hat{x}_{j}, P_{j}\right)$

Mean of a mixture:

${ }^{m} \hat{x}_{j}^{k}=\sum_{j=1}^{n} \hat{x}_{j}^{k} \mu_{j}^{k} \quad j=1, \ldots, n$

Covariance of a mixture:

$\hat{P}_{j}^{k}=\sum_{j=1}^{n} \mu_{j}^{k}\left\{P_{j}^{k-1}+\left[\hat{x}_{j}^{k}-{ }^{m} \hat{x}_{j}^{k}\right]\left[\hat{x}_{j}^{k}-{ }^{m} \hat{x}_{j}^{k}\right]^{T}\right\} \quad j=1, \ldots, n$

where Current state estimates $\hat{x}_{j}^{k},{ }^{m} \hat{x}_{j}^{k}$ Current mixture state matrices, and $\hat{P}_{j}^{k}$ Current covariance matrices.

\subsubsection{Fault Diagnose}

A threshold was set upped to detect each model probability. The Identification can screen out fault sensor signal.

if $\mu_{j}^{k} \leq$ threshold value, $f_{j}^{k}=1$, where $f_{j}^{k}$ is fault signal for each sensor.

Threshold was set to $2 \%$ in the simulation. 


\subsection{IMM Model Organized}

IMM model select Robustness and computational complexity, which can directly affects the Fault Tolerant and Diagnosis of Navigation System. To minimize complexity system states and number of model, using $2+2$ model structure 2 mixture estimates 2 autoregressive estimates, this paper uses 4 blocks of measurement mechanisms, Inertial Navigation System position information combination, aircraft dynamics model information combination and two GPS forecasting mode. These 4 blocks completely separate independent use 2 GPS measurement information and sensor information, this will maximum provide each other reference basis for the error diagnostics.
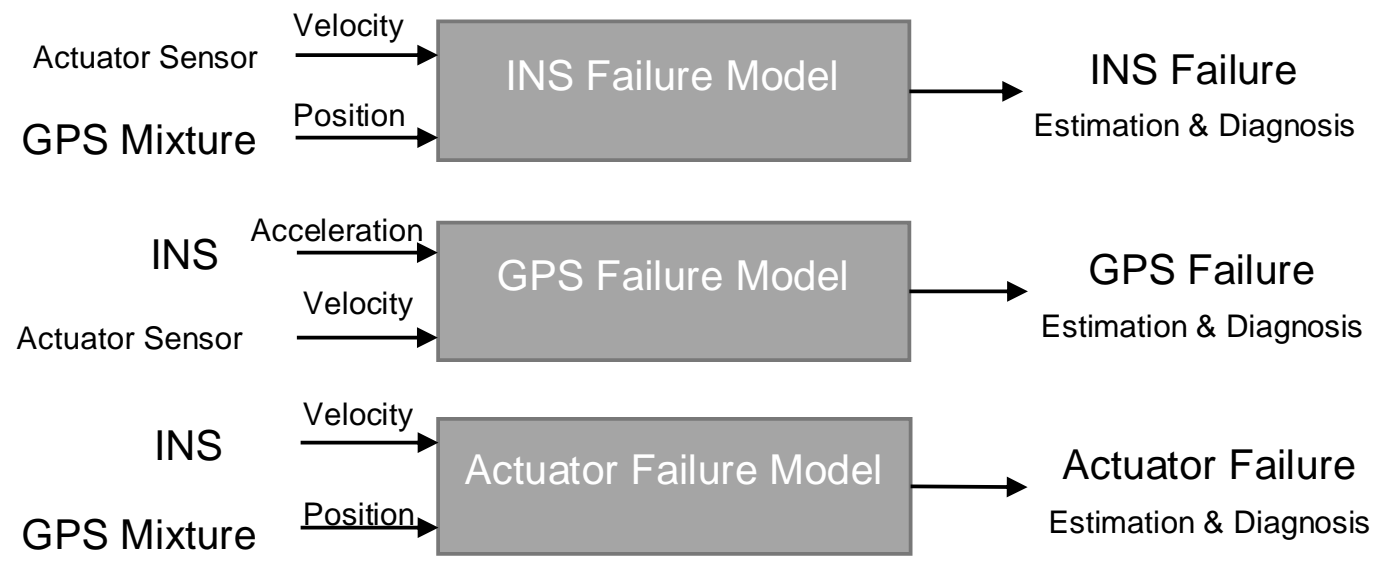

Figure 4.3 IMM Model organized structure 


\subsection{Simulation and Results}

In the DFUAV, there are five actuator sensor, two GPS, one IMU model that sensors have organized at four sensor model, all individual sensor failures were detected and properly identified. The failure scenario consists of a front rotation speed sensor failure [60sec - 100sec] and GPS 1 Signal Lost [40sec-80sec]. Both failures were properly detected and identified (see Figure 8) and the Fault Tolerant and Diagnosis of Navigation System was able to correct those error.
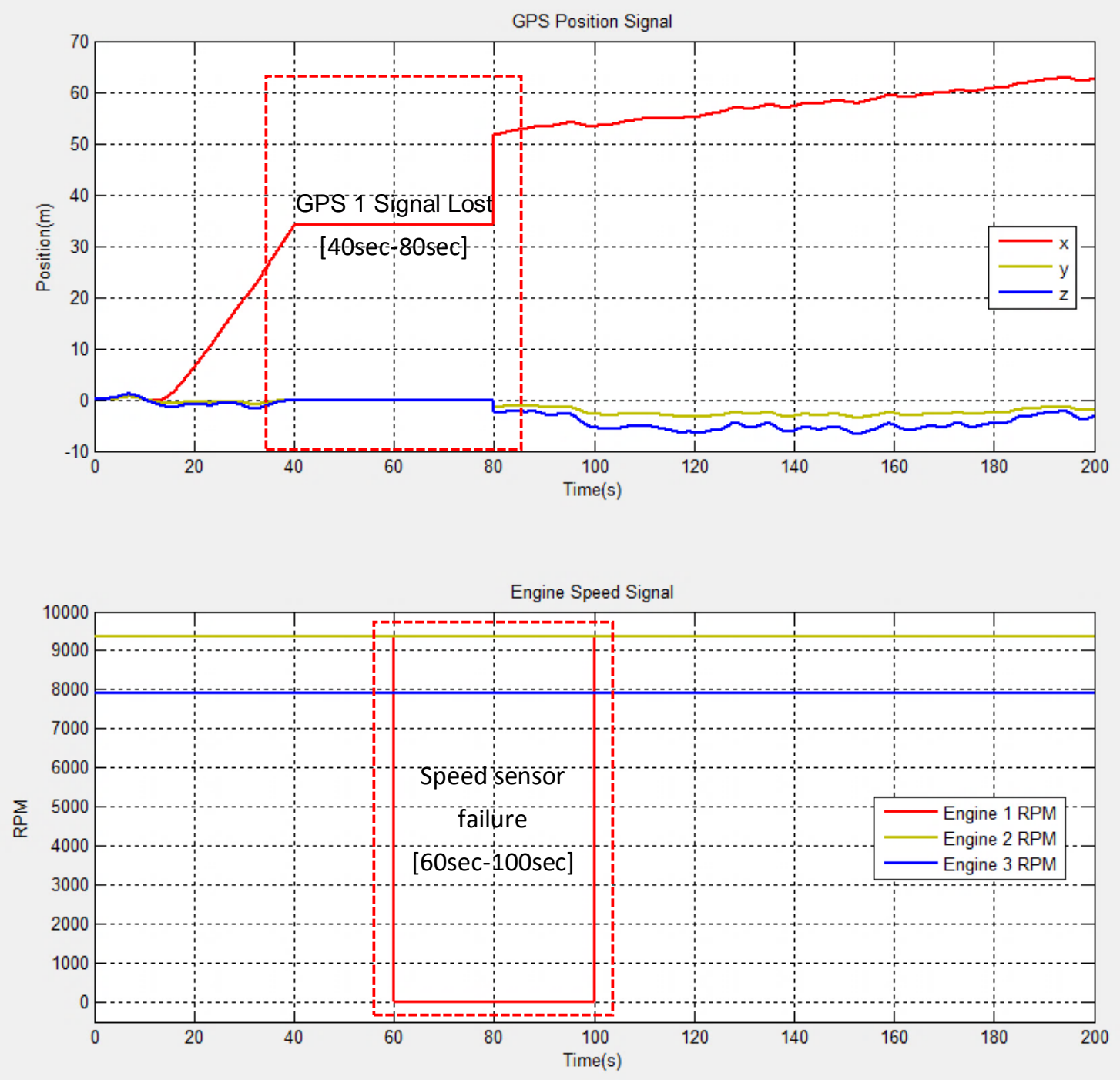

Figure 4.4 Speed sensor failures and GPS 1 signal lost describes 
The actuator sensor model probability have change to near zero. (see Figure 9).
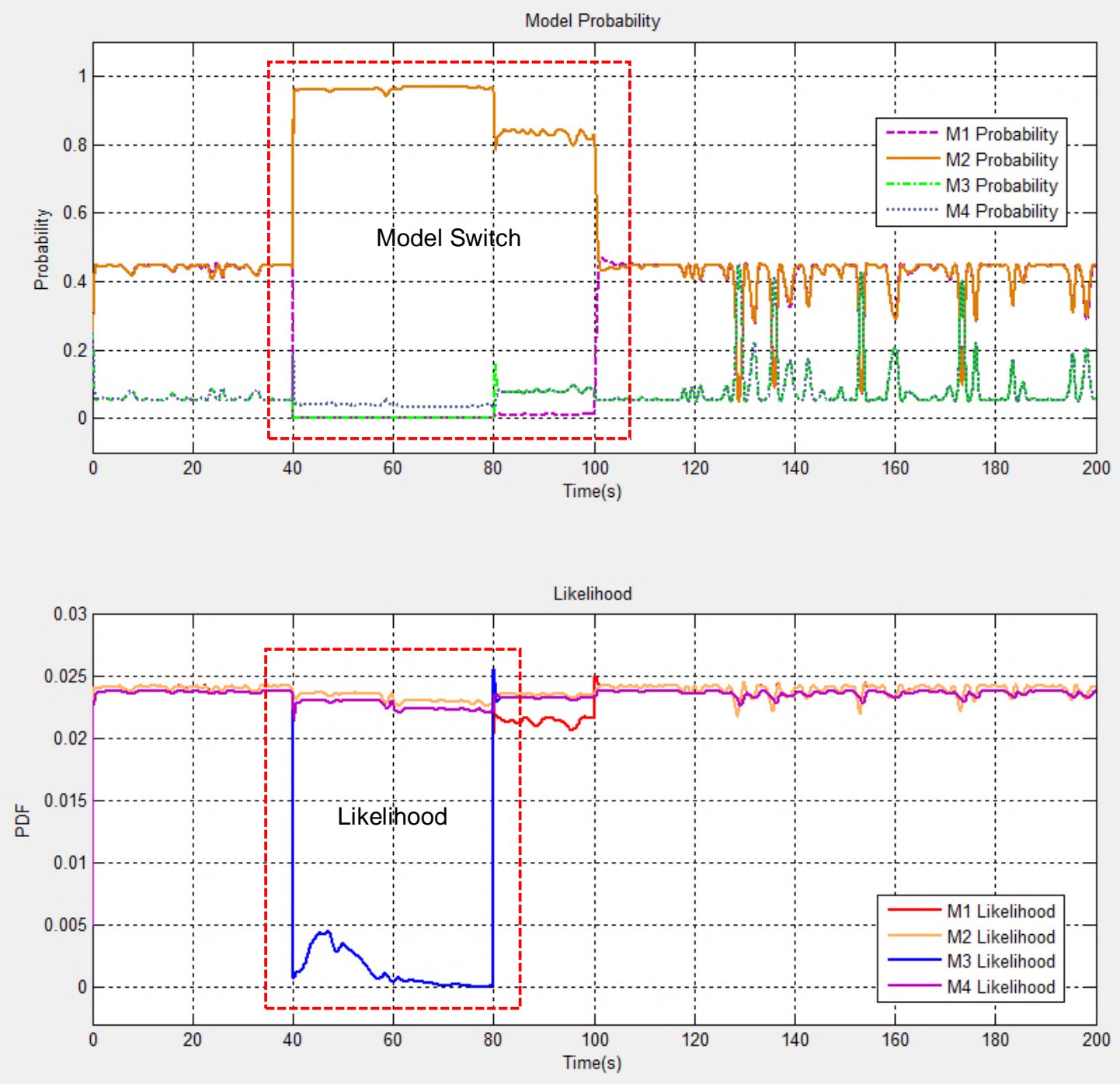

Figure 4.5 Fault-Tolerance Processing when sensor failures

The actuator sensor model probability have change to near zero. (see Figure 10). To minimize complexity system states and number of model, using $2+2$ model structure. Inertial Navigation System position information combination, aircraft dynamics model information combination using the GPS signal to comparison and recognition in Fault Tolerant and Diagnosis of Navigation System. If the GPS signal is lost, it will lost information to refer and contrast, so diagnosis system will consider Inertial Navigation System position information combination and aircraft 
dynamics model information combination may be incorrect, we can call Diagnosis Mix.
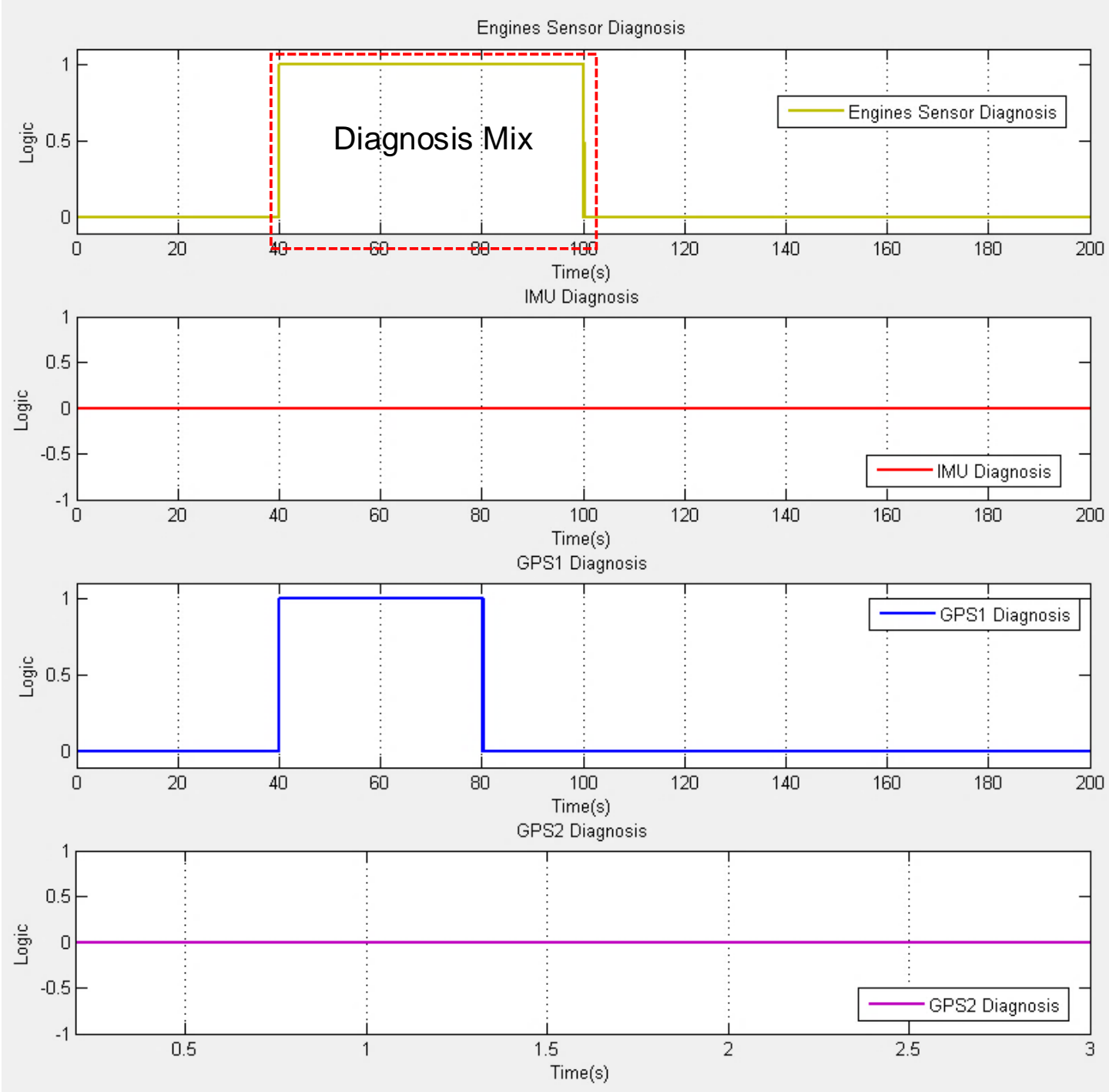

Figure 4.6 Diagnosis information

Fault Tolerant and Diagnosis of Navigation System is different whit other systems before. We using $2+2$ model structure, which can still maintain a good track performance and stability in the case of multi-sensor failure (see Figure 11). To minimize complexity system. Although there will be problems Diagnosis Mix, but did not affect the correct assessment of the Navigation System. We used rootmean-square error (RMSE) analyzed the results of the assessment in the Fault state still maintained high accuracy and stability (see Figure 12). 

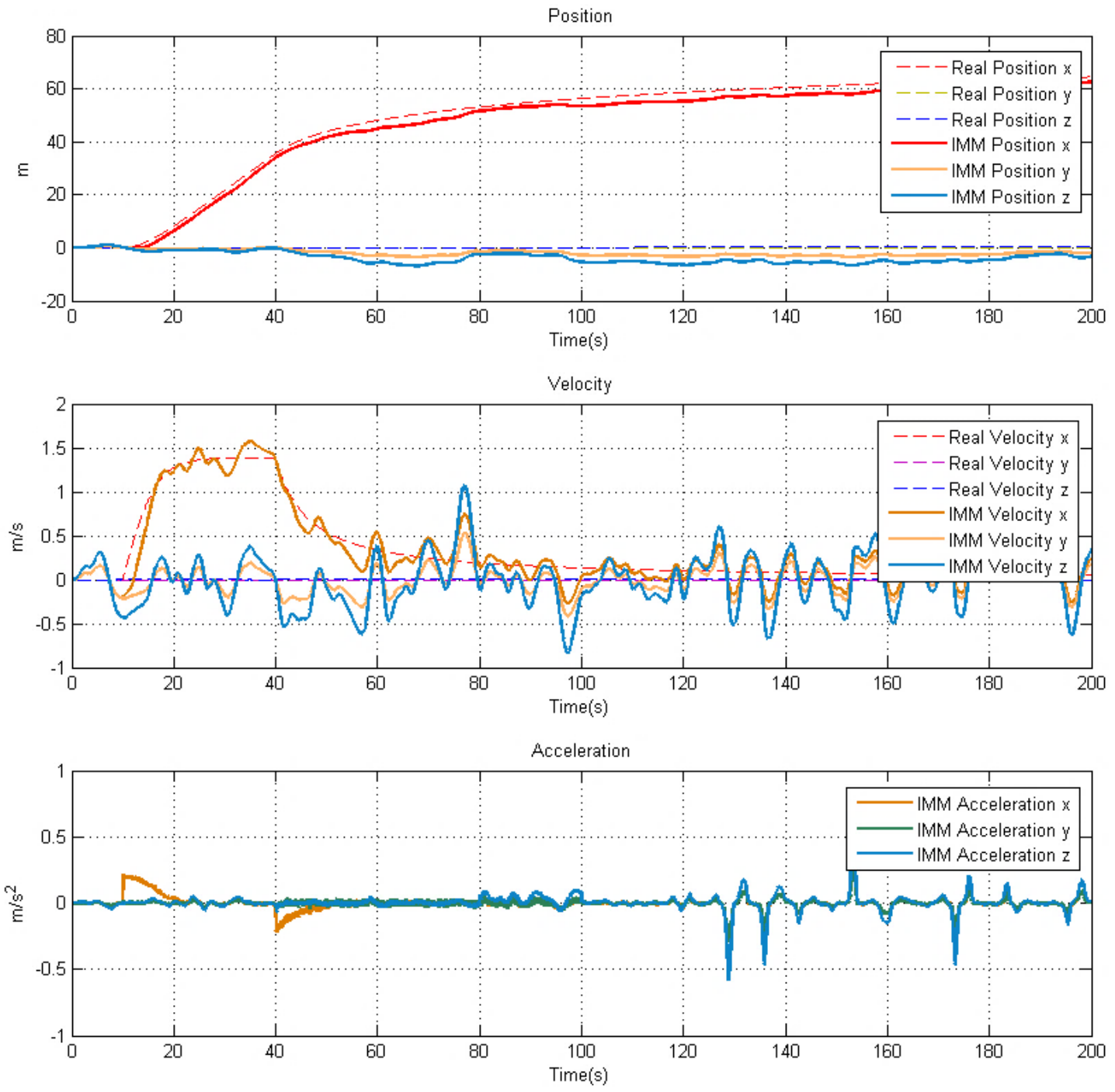

Figure 4.7 IMM filter estimation, Dotted line show actual signal 

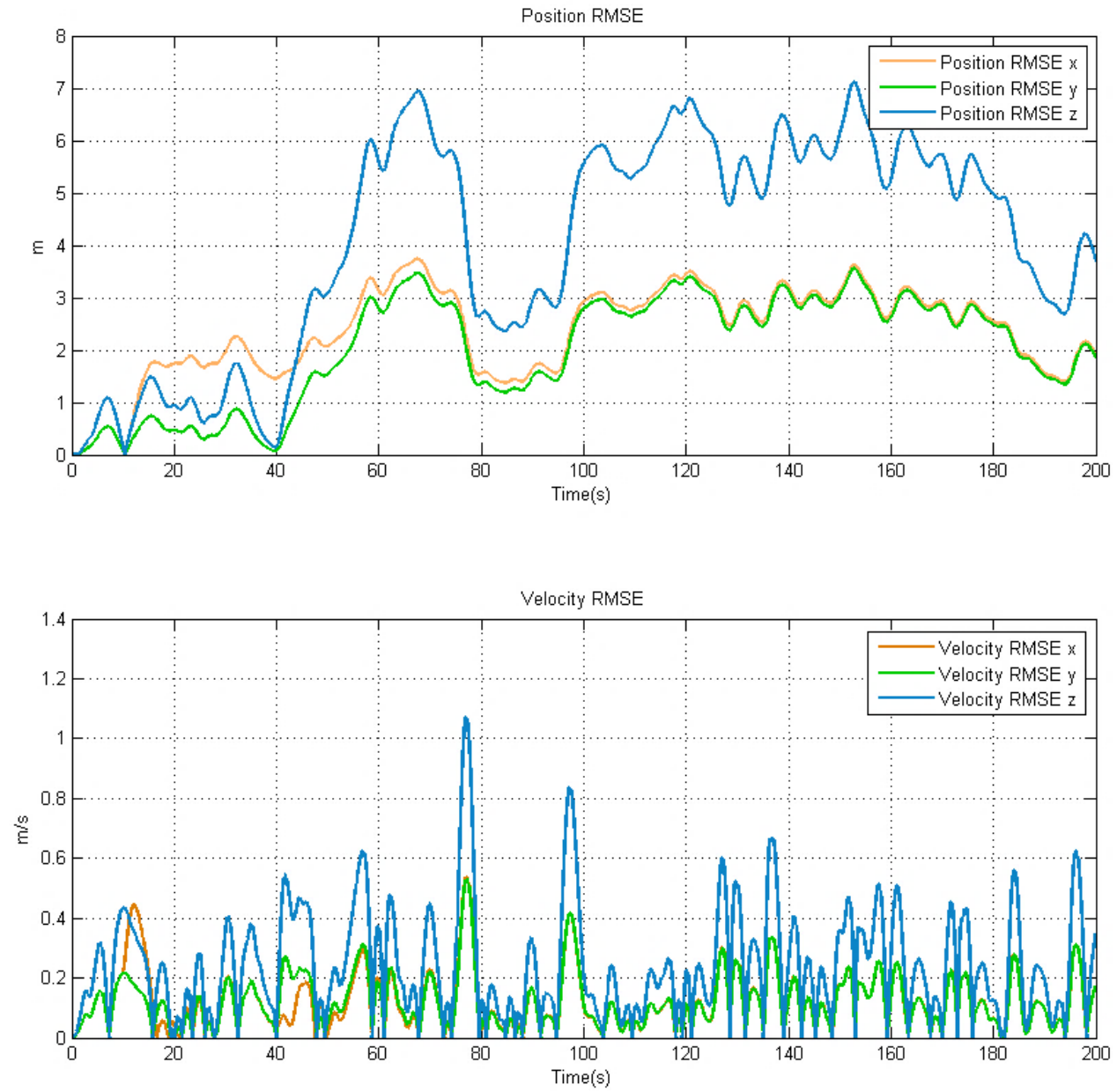

Figure 4.8 IMM filter position and velocity RMSE

Used RMSE statistical method to verify FTDF navigation system statistical characteristic. In the actual verification results show that the system in Normal state and Fault state RMSE respectively, namely, the system is stability and robustness, as shown Table 12:

Table 12 Compare RMSE in different state

\begin{tabular}{|l|l|c|c|c|c|}
\hline \multicolumn{2}{|c|}{ RMSE } & X & Y & Z & State \\
\hline \multirow{2}{*}{$\begin{array}{l}\text { Position } \\
(\mathrm{m})\end{array}$} & Normal state & 0.25034 & 0.228327 & 0.456877 & \\
\cline { 2 - 6 } & Fault state & 0.25049 & 0.228406 & 0.456635 & $\begin{array}{l}\text { Speed sensor failures [40sec-80sec] } \\
\text { GPS 1 signal lost [60sec-100sec] }\end{array}$ \\
\hline $\begin{array}{l}\text { Velocity } \\
(\mathrm{m} / \mathrm{s})\end{array}$ & Normal state & 0.015272 & 0.01487 & 0.029752 & \\
\cline { 2 - 6 } & Fault state & 0.015275 & 0.01488 & 0.02978 & $\begin{array}{l}\text { Speed sensor failures [40sec-80sec] } \\
\text { GPS 1 signal lost [60sec-100sec] }\end{array}$ \\
\hline
\end{tabular}




\section{Hardware Demo}

\subsection{Description of Hardware}

IMM Navigation system hardware is require high reliability. The system structure have a Triaxial Inertial Sensor with Magnetometer Module, a barometric pressure sensor, two GPSs and 1G Flash NAND ROM, an ARM Cortex M4 processor. The functional block diagram show in figure 5.1 .

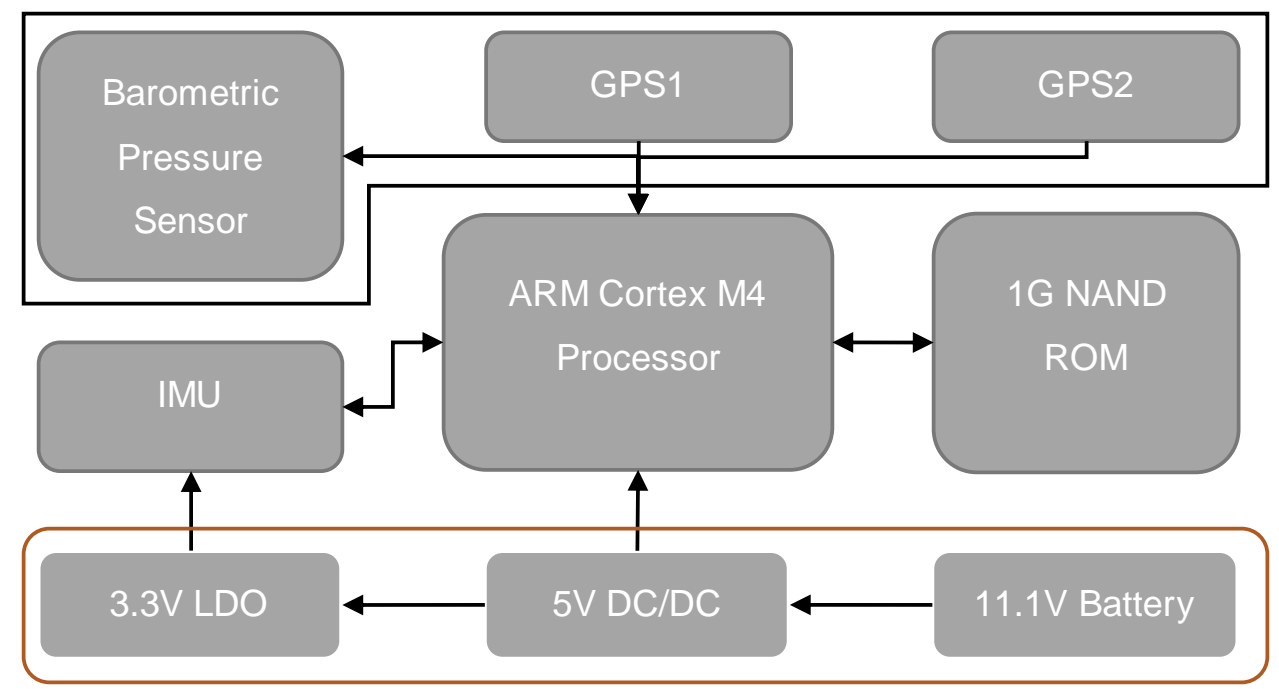

Figure 5.1 VTOL UAV Electronic System Functional Block Diagram

A lot of sensor are integrated lead to electromagnetic interference (EMI) is strangely. So the headwear filter and separate power supply have to consider in the electronic system schematic. EMI design, various interfaces are using double clock backup work, and the design is very compact. To ensure the reliability of the navigation also can reduce weight again. The navigation system are designed on a card about the size of PCB. The PCB show in Figure 5.2. 

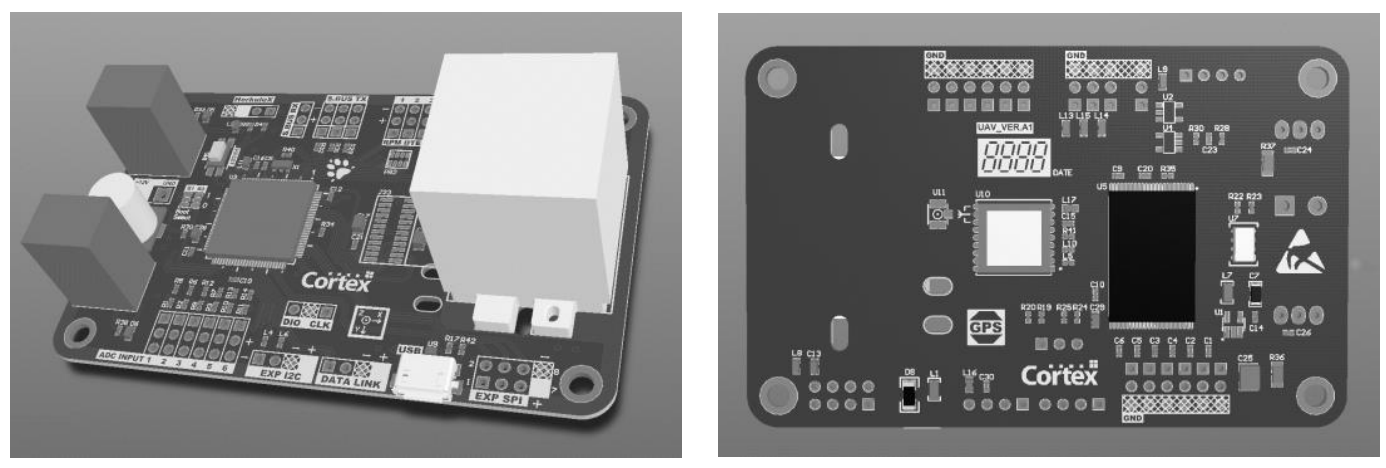

Figure 5.2 Navigation Computer

IMM navigation system was tested with a car in real environment. Consider the car cannot supply dynamic information as RPM of wheel, turn and throttle, so to car system dynamic model was canceled. Only use three model to estimate position. The structure show in Figure 5.3

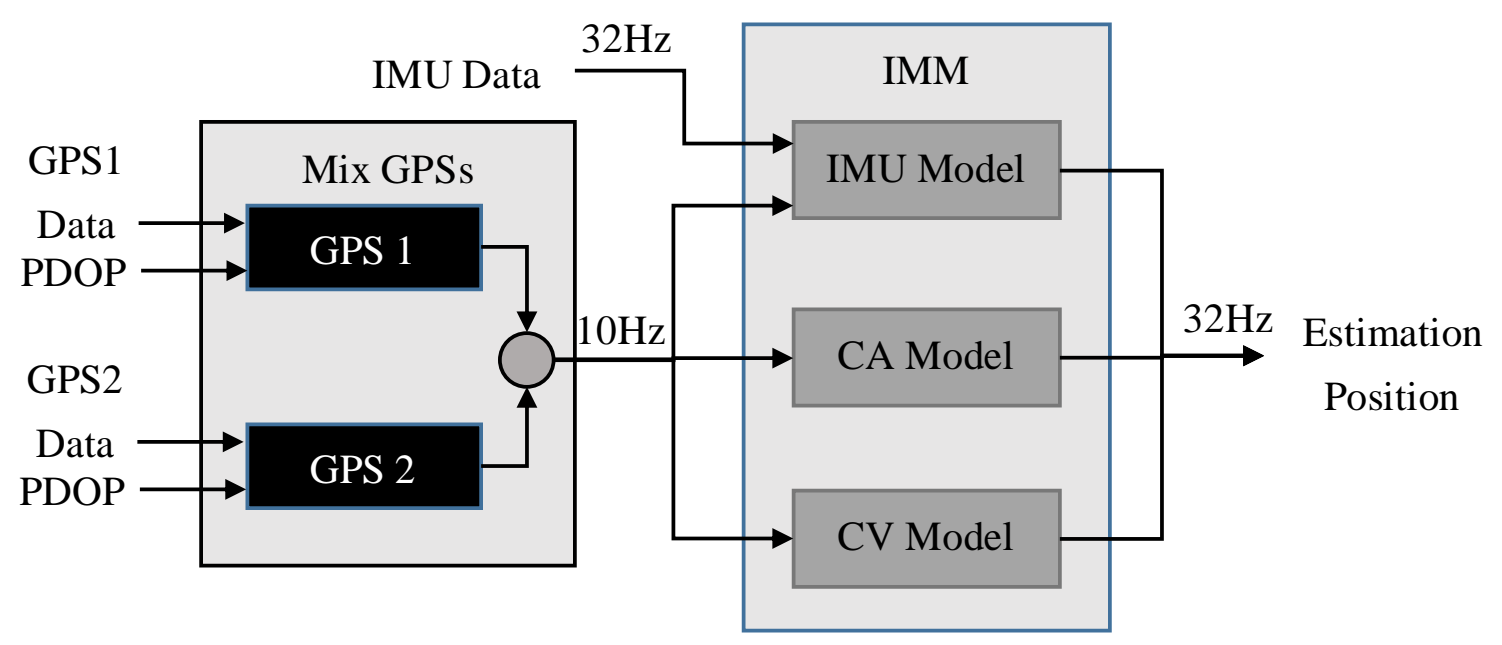

Figure 5.3 Car Navigation System Structure 


\subsection{Results}

For testing condition are made as table 13

Table 13 Testing Condition

\begin{tabular}{|c|c|c|c|c|}
\hline Testing situation & GPS1 & GPS2 & IMU & Barometric \\
\hline GPSs Signal lost & $\begin{array}{l}\text { GPS1 lost } 60 \\
\text { Sec }\end{array}$ & $\begin{array}{l}\text { GPS2 lost } 60 \\
\text { Sec }\end{array}$ & & \\
\hline Car steep turn & & & $\begin{array}{l}\text { Effect for angle } \\
\text { with lateral } \\
\text { acceleration }\end{array}$ & \\
\hline $\begin{array}{l}\text { Accelerate and } \\
\text { Decelerate }\end{array}$ & & & $\begin{array}{l}\text { Effect for angle } \\
\text { with } \\
\text { acceleration }\end{array}$ & \\
\hline Long runs & & & & $\begin{array}{l}\text { Barometric Pressure } \\
\text { Sensor bias }\end{array}$ \\
\hline
\end{tabular}

Effect for angle with lateral acceleration shown in Figure 5.4, when the pdf larger than the threshold value GD correction signal will be turn off. 

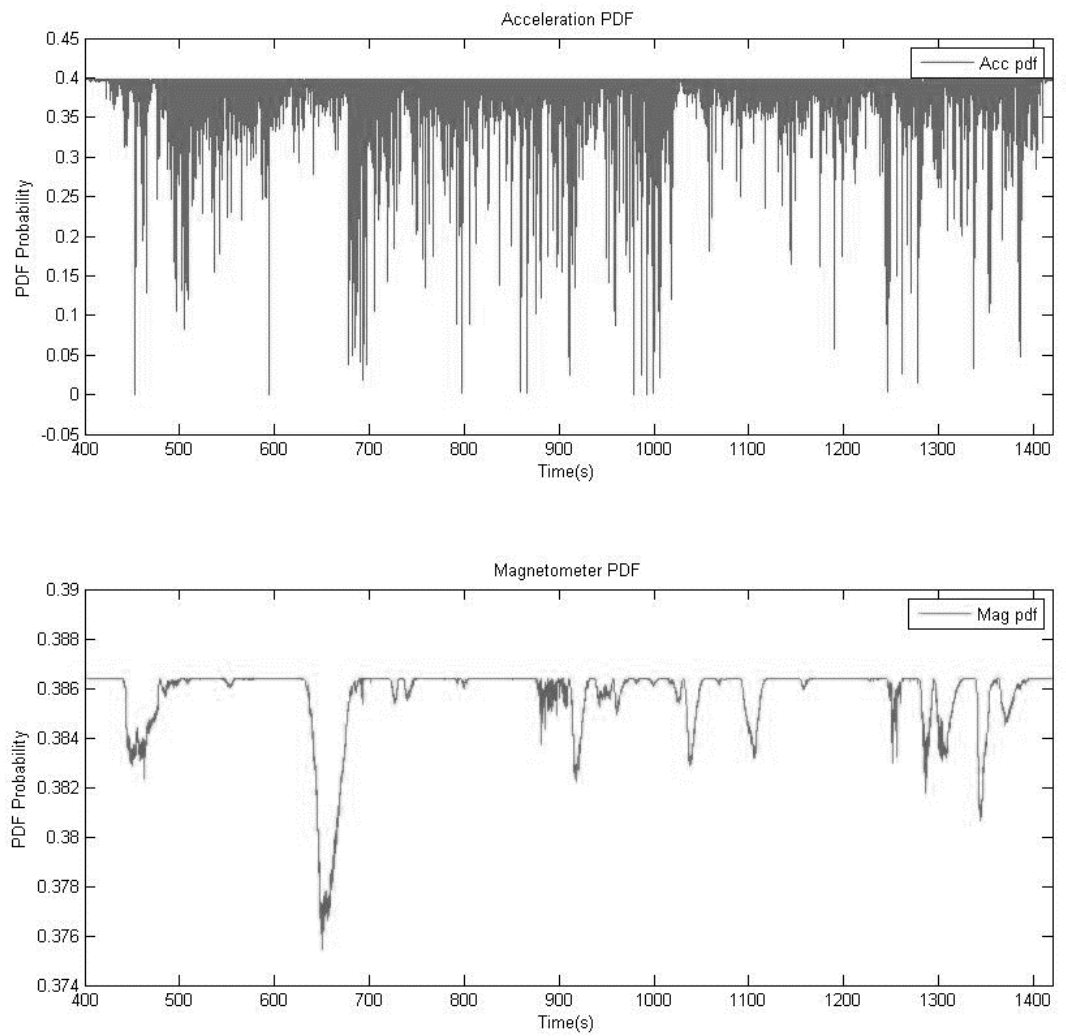

Figure 5.3 Acceleration and Magnetometers PDF 

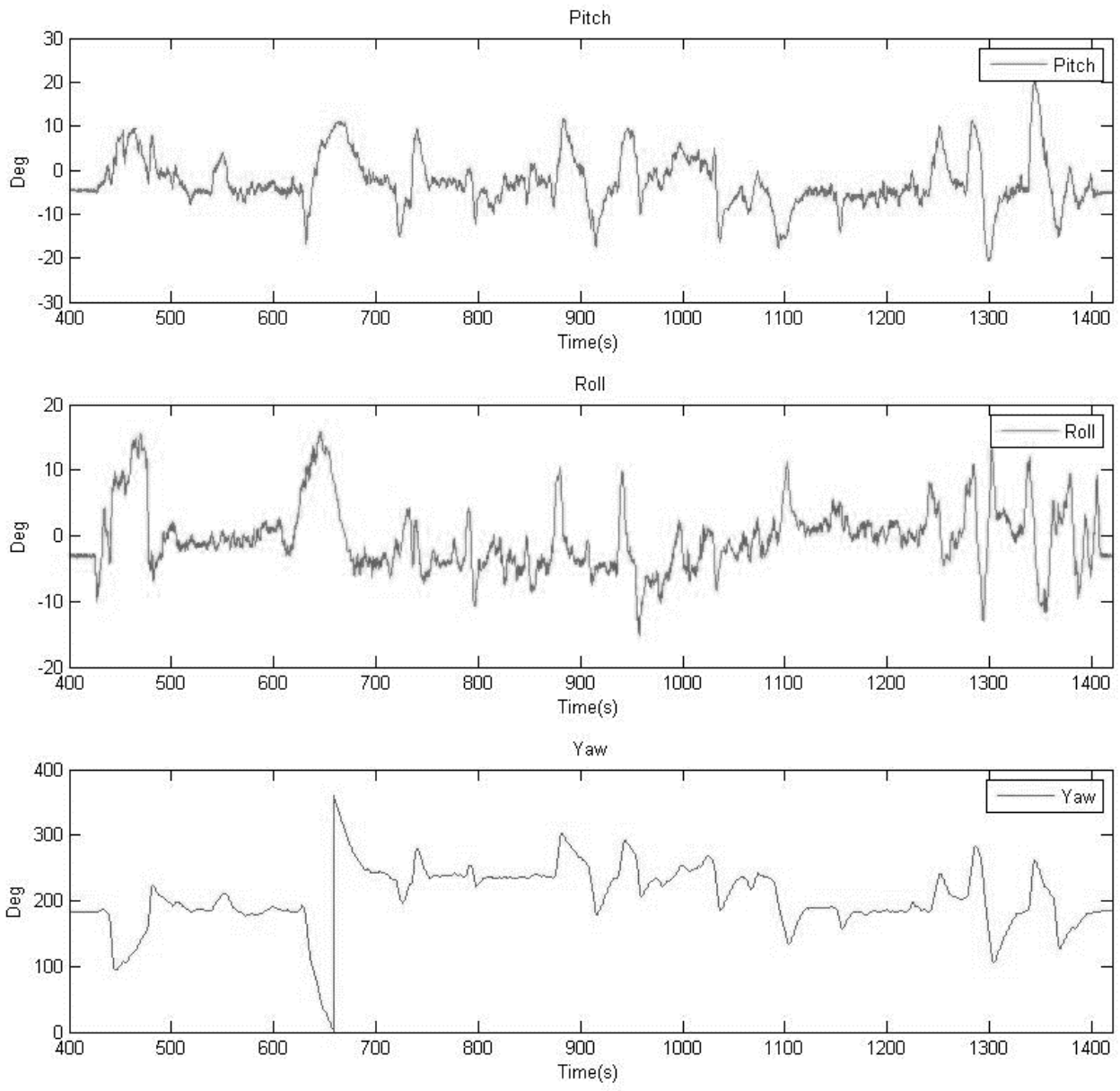

Figure 5.4 Pitch, Roll and Yaw Data from GD Filter

A simple GPB one filter was used mixture multiple GPSs signal into one output. Weight of mix depend on depend on each GPS PDOP, the figure 5.4 show GPSs PDOP likelihood and GPS switch process. 


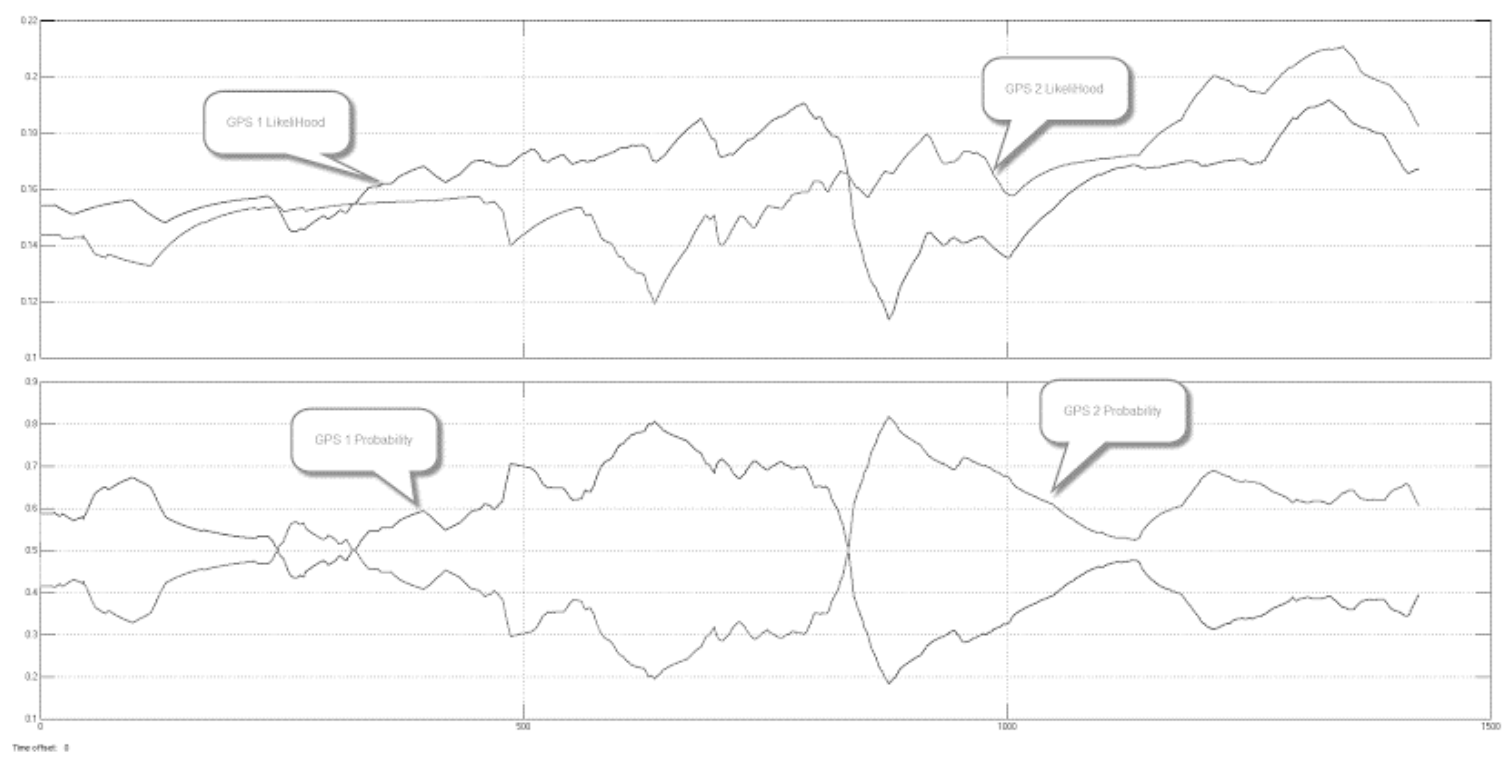

Figure 5.5 GPB 1 filter for multiple GPSs signal mixture

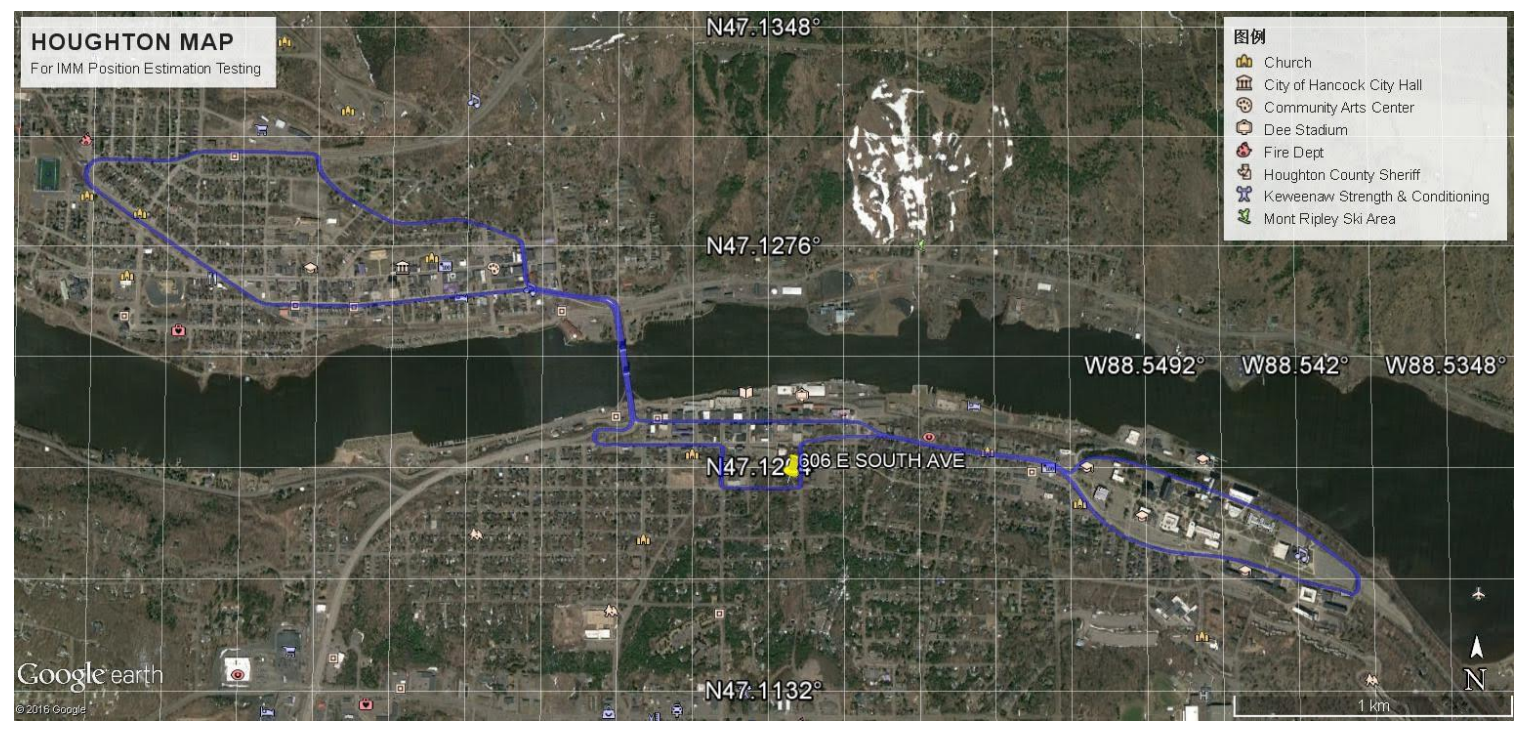

Figure 5.6 Google Map path matching with IMM estimation 


\section{Conclusion}

Nonlinear dynamics model and multi-sensor fusion of DFUAV determine the use of a system with Fault Tolerant and Diagnosis and Diagnosis robust navigation to realize evaluation to attitude and position of the aircraft. This paper focus on a FTD position system building process, including modeling model set, realize IMM fault detection, IMM model organized and verify process stability and robustness of the system. Modeling model set is to establish the corresponding mathematical model for different sensor, including dynamics model and noise model. By system identification methods and statistical variance (Allan variance) identify linear model parameters and noise parameter, using partial differential to nonlinear model to get Jacobian Matrix each state.

Realize IMM fault detection, to build the interacting multiple model (IMM) filter for Fault Tolerant and Diagnosis. IMM filter is an adaptive filter. It can automatically remove the impact of fault sensor state assessment on the system. In addition, using threshold to evaluate each model probability to identify the Fault sensor, provide to the controller for multi-model control switch, enable DFUAV can still normal flight when part of the sensor is damaged.

IMM model organize using $2+2$ model structure and 2 mixture estimates 2 autoregressive estimates, this paper uses 4 blocks of measurement mechanisms: Inertial Navigation System position information combination, aircraft dynamics model information combination and 2 prediction Model. These 4 blocks completely separate independent use 2 GPS measurement information and sensor information to minimize complexity system states and number of model.

To verify system stability and robustness, the processing has include software simulation and hardware verification. Mainly on account of the system response in case of occurrence which is sensor failed. Used RMSE statistical method to verify FTDF navigation system statistical characteristic. In the actual verification results show that the system in Normal state and Fault state RMSE respectively, namely, the system is stability and robustness, as shown Table 14:

Table 14 Compare RMSE in different state

\begin{tabular}{|c|c|c|c|c|c|}
\hline \multicolumn{2}{|c|}{ RMSE } & X & Y & Z & State \\
\hline Position (m) & Normal state & 0.25034 & 0.228327 & 0.456877 & \\
\hline
\end{tabular}




\begin{tabular}{|c|c|c|c|c|c|}
\hline & Fault state & 0.25049 & 0.228406 & 0.456635 & $\begin{array}{c}\text { Speed sensor failures [40sec-80sec] } \\
\text { GPS 1 signal lost [60sec-100sec] }\end{array}$ \\
\hline $\begin{array}{c}\text { Velocity } \\
(\mathrm{m} / \mathrm{s})\end{array}$ & Normal state & 0.015272 & 0.01487 & 0.029752 & \\
\cline { 2 - 6 } & Fault state & 0.015275 & 0.01488 & 0.02978 & $\begin{array}{c}\text { Speed sensor failures [40sec-80sec] } \\
\text { GPS 1 signal lost [60sec-100sec] }\end{array}$ \\
\hline
\end{tabular}

\subsection{Recommended Further work}

- Continue to optimize dynamic model and navigation system noise model by more testing.

- GD Filter will be improve by Conjugate Gradient Method or Levenberg-Marquardt Algorithm

- To design a Multi-Model Optimal Controller (MMOC) cooperate with IMM Filter.

- Rebuild Ducted Fan UAV structure base on Air dynamics research. 


\section{References}

[1] M. G. Ferguson, “Global Positioning System (GPS) Error Source Prediction," DEPARTMENT OF THE AIR FORCE AIR UNIVERSITY, 2000.

[2] R. B. Langley, “Dilution of Precision,” GPS World, 2011-10-12.

[3] Frequency, IEEE Standards Coordinating Committee 27 on Time and, "IEEE Standard Definitions of Physical Quantities for Fundamental Frequency and Time Metrology - Random Instabilities," IEEE-SA Standards Board, Approved 26 March 1999.

[4] H. Hou, “Modeling Inertial Sensors Errors Using Allan Variance,” UCGE Reports, September 2004.

[5] H. W. Zhao, "Development of a Dynamic Model of a Ducted Fan VTOL UAV," RMIT University, August 2009.

[6] S. O. Madgwick, “An efficient orientation filter for inertial and inertial/magnetic sensor arrays,” April 30, 2010.

[7] X. R. L. T. K. Yaakov Bar-Shalom, Estimation with Applications to Tracking and Navigation, John Wiley \& Sons, Apr 5, 2004..

[8] A. S. a. S. S. Jouni Hartikainen, “Optimal Filtering with Kalman Filters and Smoothers a Manual for the MATLAB toolbox EKF/UKF,” 2011.

[9] R. Pitre, “A Comparison of Multiple-Model Target Tracking,” University of New Orleans, New Orleans, 2004.

[10] R. R. P. V. P. J. X. R. Li, “ “A comparative study of multiple-model algorithms for maneuvering target tracking,” University of New Orleans, New Orleans, 2006.

[11] X. R. L. a. V. P. Jilkov, "A Survey of Maneuvering Target Tracking-Part V: Multiple-Model Methods," IEEE Trans. Aerospace and Electronic Systems, 2005. 
[12] R. S. a. GerdWanielik, “Unifying Bayesian Networks and IMM Filtering for Improved Multiple Model Estimation,” 12th International Conference on Information Fusion Seattle, pp. 810-817, 6 July 2009.

[13] A. F. Genovese, “The Interacting Multiple Model Algorithm for Accurate State Estimation of Maneuvering Targets," JOHNS HOPKINS APL TECHNICAL DIGEST, VOLUME 22, NUMBER 4 (2001) , pp. 614-623, 2001.

[14] R. E. F. H. B. Seham Mouawad Aly, “Extended Kalman Filtering and Interacting Multiple Model for Tracking Maneuvering Targets in Sensor Netwotrks,” 13th International Conference on AEROSPACE SCIENCES \& AVIATION TECHNOLOGY, pp. 26-28, May 2009.

[15] D. Reynolds, “Gaussian Mixture Models,” MIT Lincoln Laboratory, Lexington.

[16] G. W. a. G. Bishop, “An Introduction to the Kalman Filter,” University of North Carolina, July 24, 2006.

[17] C. Rago, "Failure detection and identification and fault tolerant control using the IMM-KF with applications to the Eagle-Eye UAV," Proceedings of the 37th IEEE Conference on., pp. 4208-4213, December 1998. 